\title{
Understanding the Novice Decision-Making Process in Forensic Footwear Examinations: Accuracy and Decision Rules
}

\author{
Madonna A. Nobel \\ mnobel@mix.wvu.edu
}

Follow this and additional works at: https://researchrepository.wvu.edu/etd

Part of the Multivariate Analysis Commons, Other Physical Sciences and Mathematics Commons, and the Science and Technology Studies Commons

\section{Recommended Citation}

Nobel, Madonna A., "Understanding the Novice Decision-Making Process in Forensic Footwear Examinations: Accuracy and Decision Rules" (2018). Graduate Theses, Dissertations, and Problem Reports. 3692.

https://researchrepository.wvu.edu/etd/3692

This Thesis is protected by copyright and/or related rights. It has been brought to you by the The Research Repository @ WVU with permission from the rights-holder(s). You are free to use this Thesis in any way that is permitted by the copyright and related rights legislation that applies to your use. For other uses you must obtain permission from the rights-holder(s) directly, unless additional rights are indicated by a Creative Commons license in the record and/ or on the work itself. This Thesis has been accepted for inclusion in WVU Graduate Theses, Dissertations, and Problem Reports collection by an authorized administrator of The Research Repository @ WVU. For more information, please contact researchrepository@mail.wvu.edu. 
2018

Understanding the Novice Decision-Making Process in Forensic Footwear Examinations: Accuracy and Decision Rules

Madonna A. Nobel

Follow this and additional works at: https://researchrepository.wvu.edu/etd

Part of the Multivariate Analysis Commons, Other Physical Sciences and Mathematics Commons, and the $\underline{\text { Science and Technology Studies Commons }}$ 


\title{
Understanding the Novice Decision-Making Process in Forensic Footwear Examinations: Accuracy and Decision Rules
}

\author{
Madonna Nobel \\ Thesis submitted \\ to the Eberly College of Arts and Sciences \\ at West Virginia University \\ in partial fulfillment of the requirements for the degree of \\ Master of Science in \\ Forensic \& Investigative Science \\ Jacqueline Speir, Ph.D., Chair \\ E. James Harner, Ph.D. \\ Lucas Mentch, Ph.D. \\ Department of Forensic \& Investigative Science \\ Morgantown, West Virginia \\ 2018
}

Keywords: data mining, rough set theory, forensic footwear evidence, footwear examination, forensic impression evidence

Copyright 2018 Madonna Nobel 


\title{
ABSTRACT \\ Understanding the Novice Decision-Making Process in Forensic Footwear Examinations: Accuracy and Decision Rules
}

\begin{abstract}
Madonna Nobel
The reproducibility of experienced-based forensic pattern interpretation is founded on the notion that domain-specific knowledge can be successfully distributed and applied among experts within a group. This assumption persists, even when the examination is complicated by variations in case circumstances, such as impression clarity and totality, as well as media, substrate, collection mechanism and enhancement. While it is further theorized that many of these factors (as well as additional confounding factors) are at play during an examination, the manner and extent to which these sources of variability affect the examination of footwear evidence remain unclear. In order to explore this hypothesis, a data mining technique called dominance-based rough set approach (DRSA) was applied to characterize the novice examiners decision-making process, due to its ability to capture useful information from a set of hybrid data with latent preference orders and discover knowledge in the form of decision rules. Through this approach, two objectives were addressed: the identification of factors that affect footwear examination and conclusions within the novice group, and the evaluation of decision rule quality as a function of support, strength, certainty and lift factors.

The results of the study showed that in general, novice examiners case assessments were found to be outside the acceptable conclusion range more than $50 \%$ of the time, with general tendencies to assign ambiguous conclusions, such as "limited association of class characteristics" and "lacks sufficient detail," rather than more definitive ones such as "identification" or "exclusion." When assessments were further explored using DRSA, 23 decision rules were induced (13 certain and 10 possible). Of the 13 certain rules, $75 \%$ of the induced rules were dominated by the examiners background, rather than case attributes, and $50 \%$ of the possible rules indicated that media type was a prevalent factor in the examiners determination of similarity/dissimilarity, as they attempted to interpret media-substrate interaction and reconcile this interpretation with SWGTREAD conclusion guidelines. Only when examiner attributes were excluded from the analysis, forcing the induction of rules based on case attributes only, did case-based features become prominent, but only with very low rule-support. In the second phase of work related to this project, the nature and type of rules induced based on expert assessments will be examined and compared to those generated from this novice set in order to compare and interpret the manner in which domain-specific knowledge dominates induced rules.
\end{abstract}




\section{Acknowledgements}

Portions of this project were supported by Award No. 2016-DN-BX-0152, awarded by the National Institute of Justice, Office of Justice Program, U.S. Department of Justice, and the Lucas Grant, awarded by the Forensic Sciences Foundation, American Academy of Forensic Sciences (AAFS). The opinions, findings, conclusions and recommendations expressed in this document are those of the author and do not necessarily reflect those of the Department of Justice nor the American Academy of Forensic Sciences.

Foremost, I would like to express my sincere gratitude to my advisor Dr. Jacqueline Speir for her continuous support, patience, enthusiasm and immense knowledge. Her guidance helped me throughout the research and writing of this thesis.

Besides my advisor, I would like to thank the rest of my research committee: Dr. James Harner and Dr. Lucas Mentch, for their insightful comments and encouragement, which inspired me to widen my perspective.

I would also like to thank Nicole Richetelli and Renuka Watalingam for their help in fine-tuning my project, and last but not least, the participants of this project for their time and effort in completing the study. 
This work is dedicated to my family. 


\section{Contents}

\begin{tabular}{lll}
\hline 1 & Introduction & 1
\end{tabular}

1.1 Literature Review . . . . . . . . . . . . . . . . . . . . 2

1.2 Theoretical Challenges in Project Design . . . . . . . . . . . . . . . . . 3

1.3 Overview of Proposed Data Analysis Technique . . . . . . . . . . . . . . . . 5

1.4 Goals of the Study . . . . . . . . . . . . . . . . . . . . 9

2 Material and Methods 10

2.1 Materials . . . . . . . . . . . . . . . . . . . . . . . 10

2.2 Participants . . . . . . . . . . . . . . . . . . . . . . . 11

2.3 Case Analysis . . . . . . . . . . . . . . . . . . . . . . . . . 12

2.4 Rule Induction $\ldots \ldots \ldots \ldots \ldots$. . . . . . . . . . . . . . . . . . . . . . . 12

$\begin{array}{lll}3 & \text { Results and Discussion } & 13\end{array}$

3.1 Overview of Data . . . . . . . . . . . . . . . . . . . . . 13

3.2 Decision Rules $\ldots \ldots \ldots \ldots \ldots \ldots$. . . . . . . . . . . . . . . . . 17

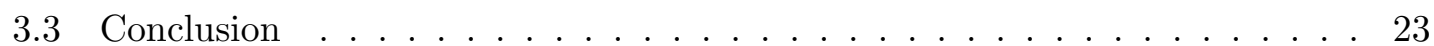

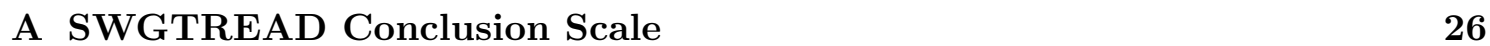

\begin{tabular}{ll}
\hline B SWGTREAD Footwear Terminology & 30
\end{tabular}

\begin{tabular}{|ll}
\hline C DRSA Terminology & 44
\end{tabular}

\begin{tabular}{|ll}
\hline D Case Images & 45
\end{tabular}

\begin{tabular}{lll}
\hline E Background Survey & 66
\end{tabular}

\begin{tabular}{lll}
\hline F & GUI & 70
\end{tabular}

\begin{tabular}{ll}
\hline G Sample Data Tables & 77 \\
\hline
\end{tabular} 


\section{Introduction}

Footwear examination is a service offered at most laboratories in the United States [1]. When detected and properly collected, this type of forensic evidence can assist in providing investigative leads, event reconstruction, source exclusion and even source identification (within the scientific confines permitted when using this colloquial term). Given the probative value of these possible outcomes, it is important to understand how footwear evidence is evaluated and compared, as well as the factors that contribute to the forensic comparative scientist's ability to recognize and properly interpret both agreements and disagreements that may be present between questioned and exemplar impressions. This invariably requires an understanding of the degree of similarity that can be expected between close non-matches and the degree of dissimilarity that can result when known matches are generated under dynamic conditions (with variable media and substrates). In other words, an analyst has to take into account the features of a questioned print and a known exemplar, including the potential effects of the deposition, recovery, and enhancement of the impression at the crime scene, influenced by his or her internalized knowledge and subject matter expertise, before any judgment regarding the evidence's association to the case can be determined. Given this complexity, it remains unclear how internal factors (such as training, experience, and certification), in conjunction with external factors (such as impression type, quality, clarity, and totality), either singularly or in combination, impact both the comparative process and the final conclusion(s) proffered by the examiner.

With the above in mind, and in order to assess the range of variables that may impact the decision making process associated with forensic footwear examinations, a twophase investigative process was initiated. The first phase attempted to define the baseline variability in conclusions when questioned and test impressions were evaluated by 'nonexperts.' This group was comprised of individuals with formal scientific education and extensive forensic science knowledge, but in the absence of meaningful domain-specific expertise and experience in footwear examinations. The second phase of the project was to assess the accuracy and variability in conclusions when the same dataset was examined by 'experts,' or practitioners with variable backgrounds (in terms of training, education, and experience), but meaningful domain-specific expertise in actual forensic footwear examinations. The remainder of this paper reviews the existing seminal research that inspired this effort, the manner in which this study differs from past work, a complete description of project design (a combination black-box/white-box approach), data analysis protocols, 
and the results associated with phase one.

\subsection{Literature Review}

Research supporting the foundational validity of feature-comparison methods is of tremendous importance to the forensic pattern-analysis community. However, the methods and outcomes associated with many forensic comparative sciences (including footwear) have been recently challenged [2], stimulating additional pattern-related research aimed at quantifying error rates, determining sources of variability, and automating feature-comparison analyses.

In footwear analysis specifically, much of the research effort has been centered on exploring the discriminating potential of footwear evidence,(i.e., physical evidence), with a minor focus on consensus and variation among examiners when presented with the same evidence. In fact, research aimed at understanding the expert decision making process in the field of forensic footwear analysis is largely limited to two influential studies, performed almost 20 years apart; the first by Majamaa and Ytti in 1996 [3], and the second by Hammer et al. in 2013 [4. Most certainly, this scarcity in investigation has raised several questions concerning reliability, compounded by the fact that the existing inquiries differed in both intended audience and project design. Despite this, as well as the evolution of the field over time, the existing studies have provided insight into the challenges associated with this type of research. For example, the Majamaa and Ytti study (1996) was conducted internationally [3], which required some laboratories to translate the provided conclusion scale into their native languages, which in turn, may have lead to artificial or erroneous variation based on linguistical differences. Furthermore, the authors did not provide a standardized interpretation for each outcome/conclusion, and instead, allowed each analyst to individually define the criteria (i.e., the type of features that must be present, and the degree of similarity/dissimilarity expressed by each) associated with a specific conclusion (e.g., "probable," "very probable," "identification," etc.) [3]. Although this ensured that the research was as much like casework as possible, differences in laboratory policies may have increased variation in the research methodology, which in turn may have added to the variability in the experts' final determinations. For example, participants from some laboratories were not permitted to reach a positive identification unless the shoe was submitted for assessment, which was not an option for this particular study 3$]$.

The Hammer et al. (2013) study was modeled after Majamaa and Ytti's work, with two notable differences. First, the targeted participants were examiners located in North America [4], and second, these participants were expected to use the seven-category conclusion scale published by the Scientific Working Group for Shoeprint and Tire Tread Evidence (SWGTREAD), which required the examiner to report his or her conclusion using one of the following verbal descriptions: "unsuitable," "exclusion," "probably did not make," "inconclusive," "could have made," "probably made" or "identification" [5] (aside: this scale was updated in 2013, but after the Hammer et al. (2013) study was complete, 
and the permitted verbal descriptions are now "lacks sufficient detail," "exclusion," "indications of non-association," "limited association of class characteristics," "association of class characteristics," "high degree of association," and "identification" [6], Appendix A]. Compared to the former study, Hammer et al. (2013) found that experienced examiners using the standardized scale had less variability in their conclusions, with the most variation occurring in a single case over the degree of confidence an examiner was willing to place on his or her final conclusion. In this case example, $82 \%$ of the examiners found the suspected shoe "probably made" the impression, $15 \%$ concluded with "could have made," and $3 \%$ chose "identification" 44. Thus, $85 \%$ of the examiners were asserting "probably made" or a more confident conclusion, while a smaller fraction were much more conservative. This is important to note, since in no way does the data indicate that examiners are at extreme ends of the spectrum with regard to their conclusions (i.e., "identification" versus "exclusion"). Instead, the differences to be understood are subtle variations in a sliding scale that lacks clear or salient boundaries, and that must be interpreted using subject matter expertise. Moreover, it is this internalized knowledge that presumably leads to variations in conclusions when examiners are presented with the same evidence; however, there is even less research on the internal and external factors that may influence this process. With this in mind, there are several fundamental questions that remain, of which two are addressed here. First, what are the factors that contribute to the decision making process, and second, what is the actual reliability associated with reported conclusions? To answer each of these questions, large scale white and black box studies (respectively) are suggested [2]. More specifically, a single gray box study can be conducted to probe each question simultaneously. However, performing a gray box study is fraught with difficulty in terms of both design, execution, and interpretation. This is especially true for footwear comparisons because the variables influencing the decision making activity are most likely (i.) numerous, (ii.) incompletely identified/understood, (iii.) non-linearly related, (iv.) partially dependent, and in some instances, (v.) nearly impossible to control for in a large scale research study. Nonetheless, steps can be taken to help foster a clearer understanding of the decision making process in forensic footwear examinations, and the following two sections independently discuss the theoretical challenges of project design and data analysis.

\subsection{Theoretical Challenges in Project Design}

The 2016 report by the President's Council of Advisors on Science and Technology (PCAST) discussing the validity of feature-comparison methods in criminal courts outlined several "best practices" criteria to be met when designing scientifically reliable studies within the pattern sciences. Of the several points articulated by this panel, three are relevant to this research: sample size, bias mitigation, and the avoidance of closed-set designs.

According to the report, an ideal black box study should have a sufficiently high participation rate, as well as a sufficiently large collection of known and questioned samples that reflect the range of variability likely to be encountered in actual casework [2. With 
regard to participation, and compared to the latent print community (with an existing black box study that has been upheld as a standard for the comparative sciences $[2,7]$ ), there are far fewer practicing footwear examiners in North America. Since the pool of experts available for participation in such a study is limited (similar studies have used 40 participants at most [3, 4] ), one of the goals of the first phase of this project was to "testrun" the case samples on a smaller pool of trained novices to establish proof-of-concept and baseline performance among non-experts. These case samples were also designed and curated to be representative of the substrates (e.g., ceramic tile, vinyl tile, linoleum tile, and paper), media (e.g., blood, dust, and wax), and quality observed in actual casework.

In addition to the sample size and sample type issue, the report recommended that the ideal study should mitigate confirmation and contextual biases [2]. Confirmation bias is defined as the tendency to interpret or perceive information in a way that confirms one's preexisting knowledge or beliefs, whereas contextual bias describes the incorporation of extraneous information in the decision making process that in turn may decrease analyst objectivity [2]. Of the two biases, confirmation bias is the most challenging to moderate in a large scale gray box study. For example, this difficulty was demonstrated in the latent fingerprint black box study by Ulery et al. (2011), where examiners were reported to have altered the features that were initially marked after comparison with a perceived matching exemplar [7]. In this study, participants were expected to adhere to the SWGTREAD examination guidelines provided, but there were no controls in place to ensure this adherence. Furthermore, none of the participants had footwear-specific training and instead had some background in either latent fingerprint or firearms examination. As such, the participants were more likely to examine and conclude in footwear cases based on their familiarity with the three-level conclusion scale (i.e., "exclusion," "inconclusive," and "identification") in the aforementioned disciplines. Despite these issues, the largest confirmation bias that exists in this study is the inclusion of case examples that involved two exemplar impressions. For example, five of the seven cases in this study presented two exemplars to the analyst for review, and it is likely that if an analyst concludes identification when comparing the questioned with exemplar one, he or she may fall victim to confirmation bias, thereby excluding the second exemplar without performing a full analysis. Thus, this limitation should be considered when interpreting results.

With regard to contextual bias, mitigation was achieved by limiting the information provided to participants to footwear manufacturer, model, and size, as well as the media, substrates, and development methods used in processing the crime scene impression. In addition, since the study is being conducted by researchers with no stake in the outcome, there is the added benefit of ensuring that neither the examiner, nor those with whom the examiner interacts on a routine basis, will have any information about the correct answers. However, this does not deter nor prevent the examiner from comparing notes with a fellow colleague or from performing a footwear verification step in addition to the examination and comparison, even when asked to refrain from doing so.

An additional criticism of previous studies is the use of closed-set designs in which the correct source is present for each questioned sample. As a result, examiners can perform 
well so long as they select the closest matching option in each sample set, effectively underestimating the false positive rate. As a result, the PCAST (2016) report recommends an open design, but without contextual bias (examiners need not be told the nature of the design). Unfortunately, adhering to an open design for a footwear black box study is somewhat problematic for two reasons. First, unlike the latent print examination threepoint scale, footwear examiners report their conclusions based on a seven-point scale, with several successive conclusion categories lacking clear demarcations (inclusion in one of two adjacent categories may represent a variation in slope, rather than a clear jump/step). Second, many footwear competency examinations span the left and right margins of the scale (exclusions and/or identification) and far less research has focused on reliability within the remaining five intermediate categories. Moreover, just because ground truth dictates that an exemplar is the source of a questioned impression, does not mean that an identification can or should be reached. The questioned impressions used in this study were collected under natural conditions, and therefore vary in both quality and clarity, as well as inherent discrimination potential (degree and type of wear, presence/absence of randomly acquired characteristics, etc.). In other words, although the research team knew which shoe created which impression, binary conclusions such as identification and exclusion were not anticipated for each and every known match (KM) and known nonmatch (KNM), respectively. Thus, this study focused most notably on reproducibility or inter-examiner variability (for both the black and white box portions of the study), while phase two will consider reproducibility, as well as accuracy as a function of adherence to SWGTREAD guidelines, rather than pure false positive and negative rates.

\subsection{Overview of Proposed Data Analysis Technique}

Given the potential variability and influencing factors that may impact examiner conclusions and interpretations, a technique is sought that is robust against incomplete and complex data. With this in mind, the rough set technique is proposed as an appropriate method to characterize and quantify the process by which comparative scientists evaluate and draw conclusions. This method is adept at deriving meaningful decision rules [8] that provide explanations of embedded trends in an intuitive and comprehensible form through "if $x$, then $y$ " statements, such as the following example: "If corresponding RACs between the questioned and known impressions are numerous (> 3) and complex, then the conclusion of 'Identification' is reached more than $90 \%$ of the time by examiners with more than 5 years of experience." Moreover, rough set theory does not rely on strict model assumptions such as normality or a priori information, other than that the input data is representative of the real world 9. Other advantages of rough set analysis include (i.) efficient extraction of hidden patterns in data, (ii.) data reduction through acquisition of minimal sets, (iii.) evaluation of data significance, (iv.) generation of decision rules, (v.) straightforward interpretation of results, and (vi.) parallel processing [9].

However, the classical rough set approach (CRSA) is primarily used with categorical data, and therefore fails to take into account scaled attributes or those that are preference- 
ordered [9] such as a footwear examiner's preference for high quality and complete (versus low quality and partial) impressions. Conversely, scaled or preference attributes (also referred to as criteria) can be easily handled by the dominance-based rough set approach (DRSA). Moreover, DRSA can extract and trace inconsistencies in decisions to missing variables, incomplete domains, and dynamic preferences, thereby illustrating the technique's ability to capture ambiguity surrounding the examiners' final conclusions.

In rough set theory (both CRSA and DRSA), each case examined by an individual can be represented as an information granule within the universe, as illustrated in Figure 1.1. Each of these granules is described by a combination of attributes, which is used to group similar cases (or objects) so as to reduce redundant information.

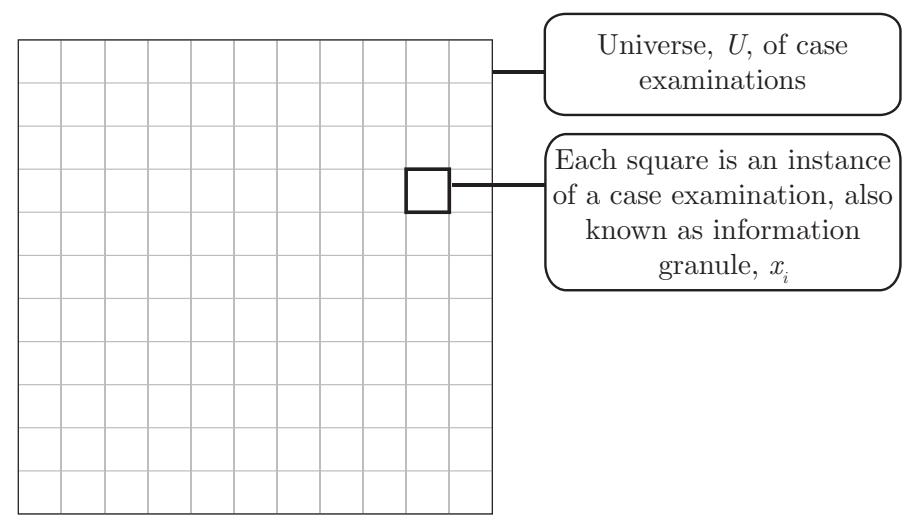

Figure 1.1: Information granule within this universe is an instance of a case examination, which includes numerous attributes, such as features related to the questioned impression (quality, totality, substrate, etc.), the known impression (size, tread design, etc.), and the examiner's background (education, experience, certification, etc.).

The dataset can also be organized into a decision table, as shown in Table 1.1, which describes Novice A's decisions for three cases. The columns labeled "Educational Background," "Impression Quality," and "Conclusion" are collectively called the attribute set. Educational background is a qualitative feature, which means that the values are categorical. Conversely, impression quality is deemed a criterion whereby the values are

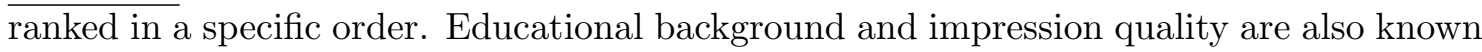
as conditional variables, in contrast to the conclusion, which is considered separately as a decision attribute in a preferentially ordered domain. A second decision table (Table 1.2 ) is created to show the decisions made by Novice B for the same cases.

In both Tables 1.1 and 1.2, each examination can be described by each feature and the particular value associated with it. This is called an information function, which essentially means a descriptor for an object (e.g., the impression quality for Case 1 is rated as good, according to Novice A in Table 1.1). Note, however, that Novice B may judge the same crime scene print differently. 
Table 1.1: Decision table for Novice A.

\begin{tabular}{cccc}
\hline Case No. & Educational Background & Impression Quality & Conclusion \\
\hline 1 & Forensics & Good & Identification \\
2 & Forensics & Poor & Lacks sufficient detail \\
3 & Forensics & Medium & Exclusion \\
\hline
\end{tabular}

Table 1.2: Decision table for Novice B.

\begin{tabular}{cccc}
\hline Case No. & Educational Background & Impression Quality & Conclusion \\
\hline 1 & Chemistry & Good & High degree of association \\
2 & Chemistry & Poor & Lacks sufficient detail \\
3 & Chemistry & Poor & Lacks sufficient detail \\
\hline
\end{tabular}

After establishing the description of each case using the attribute set, the next step is to determine which cases lead to a specific decision even though they may possess different criteria and categorical assignments. In other words, this step ascertains the "minimum" or "maximum" set of attributes that demarcate a specific decision class. Each case is now referenced by a descriptive profile $P$ that consists of attribute-value pairs that can be grouped together within the dataset. Objects sharing the same descriptors and outcomes (or decision attribute, $d$ ) are further linked together in a set, as shown in Figure 1.2 . bounded by the dashed rectangle. The set with a dotted border includes cases with the same description but not always leading to the same outcome, hence possibly leading to other outcomes. Any object found in the complement of these two sets (the shaded area) does not share the same set of attribute-values and overall evaluation. Note that if there is at least one instance found within the rectangle defined by the dotted line, but outside of the dashed rectangle, then it is called a rough set; otherwise, the set is crisp (i.e., cases that can be said with certainty to lead to one particular conclusion or another). 


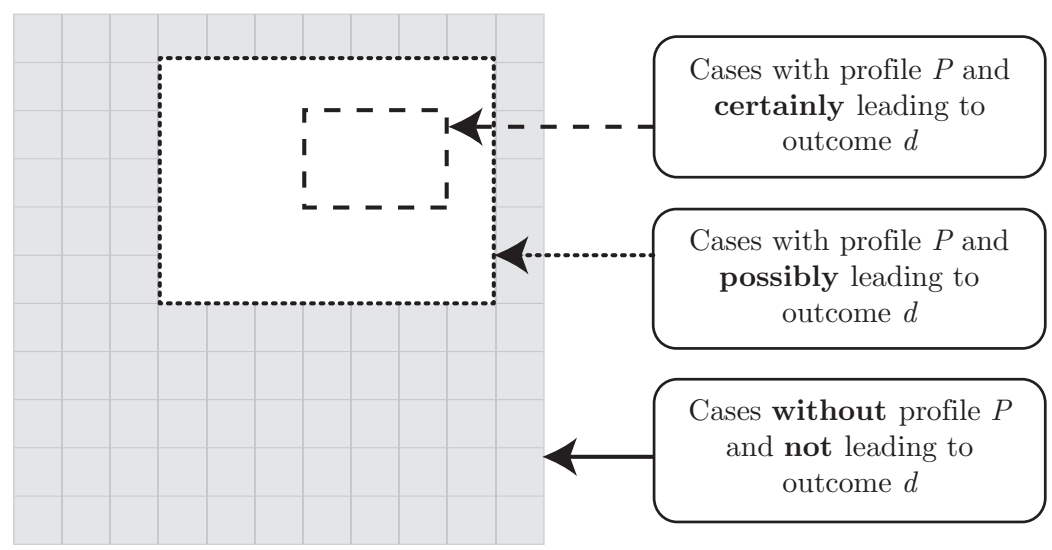

Figure 1.2: Defining class memberships with respect to conditional and decision features.

The collection of cases are defined for every decision and then used as a basis for rule induction. To derive decision rules, set $T$ (Figure 1.3 ) is determined according to the overall conclusion of interest, such as "identification," with respect to the attribute profile, $P$. The lower and upper approximations of set $T$ are then defined to form certain and possible rules, respectively. Cases that do not lead to the outcome of interest (i.e., those that are within the shaded portion of Figure 1.3) are not considered.

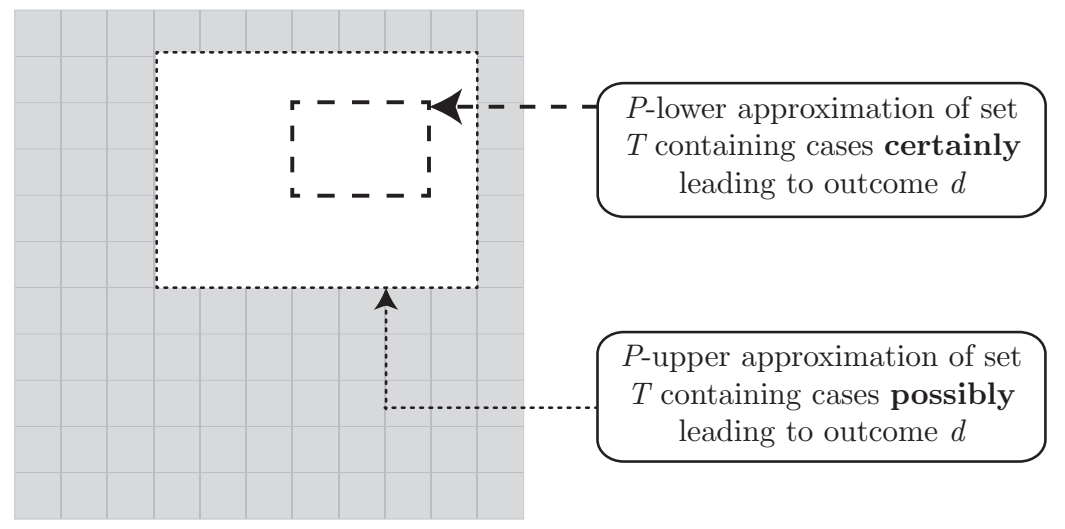

Figure 1.3: Defining lower and upper approximations of set $T$ with respect to $P$.

The resulting approximations of class membership are then used for the induction of decision rules, which come in the form of "if ..., then ..." statements. Note that these statements are not intended to imply causation, but serve as a semantic and comprehensive way of relating the features of a case to the conclusion assigned by the examiner. The consistency of the decision rules with respect to the decision table are then determined quantitatively using measures called the support $\left(\right.$ supp $\left._{x}\right)$, strength $\left(\sigma_{x}\right)$, certainty factor $\left(c e r_{x}\right)$ and lift factor $\left(\right.$ lift $\left._{x}\right)$, all of which are defined in Equations (1.1) (1.4) 10, 11. In 
each equation, the cardinality of a set is denoted as $|\cdot|, c$ refers to the condition part of the rule, and likewise, $d$ refers to the decision part of the rule.

In words, the support of a rule (Equation (1.1)) is essentially the number of cases that match both the condition and decision parts of the rule. Similarly, the strength (Equation (1.2) computes the ratio of the support to the number of cases considered in the decision table 9,12 . Alternatively, certainty of a rule (Equation (1.3)), also known as the confidence ratio, is interpreted as a conditional probability that a case $x$ has a particular conclusion $d$ given that $x$ matches the condition part of the rule, $c(x)$. In other words, the confidence ratio is a measure of certainty to which the condition implies the decision. Conversely, lift factor (Equation (1.4) is a conditional probability that a case $x$ matches the condition part of the rule, given that it has a particular conclusion $d$, which is often used to assess the value of including the rule versus excluding it.

$$
\begin{aligned}
\operatorname{supp}_{x}(c, d) & =|c(x) \cap d| \\
\sigma_{x}(c, d) & =\frac{\operatorname{supp}_{x}(c, d)}{|U|} \\
\operatorname{cer}_{x}(c, d) & =\frac{\operatorname{supp}_{x}(c, d)}{|c(x)|} \\
\operatorname{lift}_{x}(c, d) & =\frac{\operatorname{supp}_{x}(c, d)}{|d|}
\end{aligned}
$$

\subsection{Goals of the Study}

Given the advantages of the dominance-based rough set approach, this research used the aforementioned data mining technique to better discern how novice examiners interpret the pattern recognition process of footwear comparison from start to finish. In accomplishing this goal using DRSA, two additional objectives were achieved:

1. Identification of factors that affect footwear examination and conclusions via decision rule induction;

2. Evaluation of decision rule quality as a function of strength, support, certainty and lift factor; 


\section{Material and Methods}

\section{$2.1 \quad$ Materials}

In order to minimize variability in the creation of footwear impressions and exemplars, seven case studies comprising a master set were prepared. At minimum, a case study consisted of high resolution images of (i.) a crime scene-like footwear impression created from one of three different media (dust, blood or shoe polish) on one of four possible substrates (ceramic tiles, linoleum tiles, vinyl tiles or paper); (ii.) one outsole per known footwear; and (iii.) two high quality Handiprint exemplars per known. These case items were scanned at a resolution of 1200PPI using an Epson Expression 11000XL Graphic Arts scanner and then printed for mass distribution at a reproduction scale of $1: 1$, and a resolution of 1200PPI, using a Canon PIXMA PRO-1 color inkjet printer. The contents of all seven cases were then provided to the participants in both hard (physical prints) and soft copy (digital) forms (see Appendix D), allowing each participant to conduct his or her examination based on personal preference (physical side-by-side print comparisons, digital comparisons, creation of additional transparent overlays, etc.). In an ideal scenario, the actual shoes would also be available for examination (instead of only providing the physical and digital reproductions); however, this was not feasible in actual practice, which means the results of this study are bounded by the fact that all conclusions are based on examination of imagery only.

Table 2.1 shows the substrates, media and processing or enhancement method for each crime scene impression (denoted by Q). To a reasonable degree, the crime scene impressions were created in a manner that closely simulated real-world casework samples.

Table 2.1: Substrates and media for crime scene impressions. Q denotes that the item is a questioned impression sample.

\begin{tabular}{llll}
\hline Item & Substrate & Medium & Processing \\
\hline 001Q & Ceramic tile & Blood & Leucocrystal Violet (LCV) \\
002Q & Vinyl tile & Dust & Digital enhancement of gel lift \\
003Q & Ceramic tile & Blood & Leucocrystal Violet (LCV) \\
004Q & Linoleum tile & Wax & Magnetic powder and gel lift \\
005Q & Vinyl tile & Dust & Digital enhancement of gel lift \\
006Q & Paper & Dust & Digital enhancement \\
007Q & Ceramic tile & Blood & Leucocrystal Violet (LCV) \\
\hline
\end{tabular}


Table 2.2: Substrates and media for crime scene impressions. Q denotes that the item is a questioned impression sample.

\begin{tabular}{lllll}
\hline Item & Substrate & Medium & Processing & Known Footwear(s) \\
\hline $001 \mathrm{Q}$ & Ceramic tile & Blood & Leucocrystal Violet (LCV) & $001 \mathrm{~K} 1,001 \mathrm{~K} 2$ \\
$002 \mathrm{Q}$ & Vinyl tile & Dust & Digital enhancement of gel lift & $002 \mathrm{~K} 1$ \\
003Q & Ceramic tile & Blood & Leucocrystal Violet (LCV) & $003 \mathrm{~K} 1,003 \mathrm{~K} 2$ \\
$004 \mathrm{Q}$ & Linoleum tile & Wax & Magnetic powder and gel lift & $004 \mathrm{~K} 1,004 \mathrm{~K} 2$ \\
$005 \mathrm{Q}$ & Vinyl tile & Dust & Digital enhancement of gel lift & $005 \mathrm{~K} 1$ \\
$006 \mathrm{Q}$ & Paper & Dust & Digital enhancement & $006 \mathrm{~K} 1,006 \mathrm{~K} 2$ \\
$007 \mathrm{Q}$ & Ceramic tile & Blood & Leucocrystal Violet (LCV) & $007 \mathrm{~K} 1,007 \mathrm{~K} 2$ \\
\hline
\end{tabular}

In addition, the known shoes (denoted as $\mathrm{K}$ ) were selected to closely resemble the crime scene sample as much as possible in terms of class and subclass characteristics (e.g., brand, outsole design, style, and size), so as to mimic the challenge of actual casework examination (Table 2.3).

Table 2.3: Manufacturing details for each known footwear/shoe.

\begin{tabular}{lllcl}
\hline Known Footwear(s) & Manufacturer & Style & Size & Additional Details \\
\hline $001 \mathrm{~K} 1,001 \mathrm{~K} 2$ & Converse & All Star & 9 & - \\
$002 \mathrm{~K} 1$ & Nike & Lebron James & 10 & - \\
$003 \mathrm{~K} 1,003 \mathrm{~K} 2$ & Nike & Rosherun & 9 & Microcellular material \\
$004 \mathrm{~K} 1,004 \mathrm{~K} 2$ & Nike & Air Max & 10.5 & - \\
$005 \mathrm{~K} 1$ & Nike & Air Max & 11 & - \\
$006 \mathrm{~K} 1,006 \mathrm{~K} 2$ & Nike & Air Max Cage & 10 & - \\
$007 \mathrm{~K} 1$ & Under Armour & - & 11 & - \\
$007 \mathrm{~K} 2$ & Under Armour & - & 10 & - \\
\hline
\end{tabular}

\section{$2.2 \quad$ Participants}

Participant enrollment and data collection protocols were followed as approved by the West Virginia University Institutional Review Board (IRB) (Protocol No. 1602021821). Participants were recruited from a FEPAC-accredited forensic science program, and this cohort included a single undergraduate student, and six graduate students, for a total of seven participants in this proof-of-concept study. A survey was distributed to each participant and to ensure anonymity, did not elicit identifying information from the participant such as his or her name. Instead, every individual was assigned an alphanumeric ID string that served to link his or her survey responses to the case examination conducted. The questionnaire was developed to probe multiple aspects related to the participant's educational, practical, and research experience. This included questions regarding a participant's area of forensic emphasis (e.g., chemistry, biology or pattern analysis), details concerning practical experience (e.g., internships, employment, etc.), and whether the participant had conducted any pattern related research. A complete list of these questions can be found 
in Appendix E,

For each participant, information regarding his or her academic and practical background experience was organized into an information table, which includes courses taken, practical activities performed during completion of formal education, continuing education courses taught and/or attended, as well as research experience (Appendix G. Table G.1).

\subsection{Case Analysis}

The participants were then given a period of one month to complete their examinations and report their findings according to SWGTREAD guidelines [6, 13], also in Appendix A]. The novices' detailed responses for each case were collected using a graphical user interface (GUI) (Appendix F). Using the GUI, the participant was prompted to answer a series of questions, beginning with his or her evaluation of the quality of the crime scene impression (totality, clarity, and the similarity in class characteristics between the questioned and known footwear) (Appendix G. Table G.2). The participant was then asked to comment on the presence, absence, and similarity (between the questioned and known exemplars) of subclass and randomly acquired characteristics. This included the possible presence of mold defects, wear, Schallamach patterns, etc., followed by his or her assessment of the clarity, similarity, and value of each noted feature (Appendix G. Table G.3.

In order to ascertain common features marked by multiple participants, the $\mathrm{x}, \mathrm{y}$ coordinates for each marked feature were compared, and features falling within similar regions $( \pm 30$ pixels $(0.635 \mathrm{~mm})$ in either $\mathrm{x}$ or $\mathrm{y}$ direction) across different participants were then treated as a common feature while still retaining the original individual evaluations regarding the type, clarity, similarity, and strength. Features falling outside any common range were treated as standalone points. All features were then added to each participant's existing information table. Finally, the participant was prompted to make a decision regarding the case (using the aforementioned SWGTREAD seven-category scale). This information, along with any additional remarks offered by the participant regarding limiting factors (e.g., substrate-texture interaction, photographic distortion, improper lighting, improper scale position, etc.), was collated with the existing case attribute-value pairs, creating a decision table for the entire dataset. Note that the conclusion "lacks sufficient detail" is not considered part of an ordinal scale, but decisions assigned such may be included in decision table if such decisions exist in the dataset.

\subsection{Rule Induction}

Finally, rules describing how attribute-value pairs link with decisions were generated using the DOMinance-based Learnable Evolution Model (DOMLEM) algorithm developed by Greco et al. (2001) 14. To reiterate, these rules take the form of "if $x$, then $y$ " statements. However, given that there were 860 possible attribute-value pairs based on the totality of information derived from this study, a subset of attributes was selected for data processing 
moving forward (Table 2.4).

More specifically, two criteria and four qualitative attributes were selected from the background survey so as to capture the sum of the participant's experience in forensic science and pattern sciences. Additionally, one criteria and five conditional attributes were selected from the case assessments in order to capture the participant's evaluation of similarity between the exemplars and questioned impression.

Of the whole attribute set, three were considered criteria: combined educational experience, frequency in performing examination and comparison of pattern impression, and perceived clarity of the questioned impression. Nine were qualitative attributes: area of emphasis in any pattern subfield, discipline of focus during internship, tasks performed during internship related to pattern sciences in general, internship site services related to pattern sciences, media, and substrate, values of outsole design and physical sizes of design and outsole. The remaining attributes were quantitative: totality of questioned impression, number of features marked per value per case. This reduced set was used to conduct rule induction, and all generated rules were evaluated using four measures of rule quality, as described in Equations (1.1) (1.4).

Table 2.4: List of conditional attributes used to generate decision rules.

\begin{tabular}{lll}
\hline Attribute type & Background survey & Case Examination \\
\hline Criteria & Combined experience in forensic science (total number of years) & Clarity of questioned impression \\
& Examination and comparison of pattern impressions (task frequency) & \\
\hline Qualitative & Area of emphasis in any pattern-related subfield & Substrate \\
& Discipline of focus during internship (any pattern) & Medium \\
& Tasks performed during internship related to pattern sciences & Value of outsole design \\
& Internship site services related to pattern sciences & Value of physical size of design \\
& & Value of physical size of outsole \\
\hline Quantitative & & Totality of questioned impression \\
& & No. of features marked per value per case \\
\hline
\end{tabular}

\section{Results and Discussion}

\subsection{Overview of Data}

This section discusses the range of conclusions for each exemplar comprehensively, followed by the ranges for known matches and known non-matches.

Overall, there were 84 decisions reached by seven participants on 12 exemplars. Figure 3.1 shows the distribution of the range of conclusions for each comparison of the crime scene impression to known footwear. The smallest range was reported for the crime scene impression made in shoe polish/wax for exemplar $004 \mathrm{~K} 1$ where $71 \%$ of the partic- 
ipants concluded with "exclusion" and the remaining $29 \%$ reported that the questioned impression had "indications of non-association" when compared to the exemplar. This consistency in conclusion can likely be attributed to the high quality of the crime scene impression, which allowed participants to observe greater detail and arrive at a more confident conclusion.

The largest range of conclusions was reported when participants compared the questioned impression made in blood to exemplar 007K1, with results spanning from "lacks sufficient detail" (14\%), "exclusion" (29\%), "limited association of class characteristics" (29\%), "association of class characteristics" (14\%), to "high degree of association" (14\%). This suggests that some of the participants may have encountered some difficulty in reconciling their determination of the degree of association and their understanding of the SWGTREAD conclusion scale.

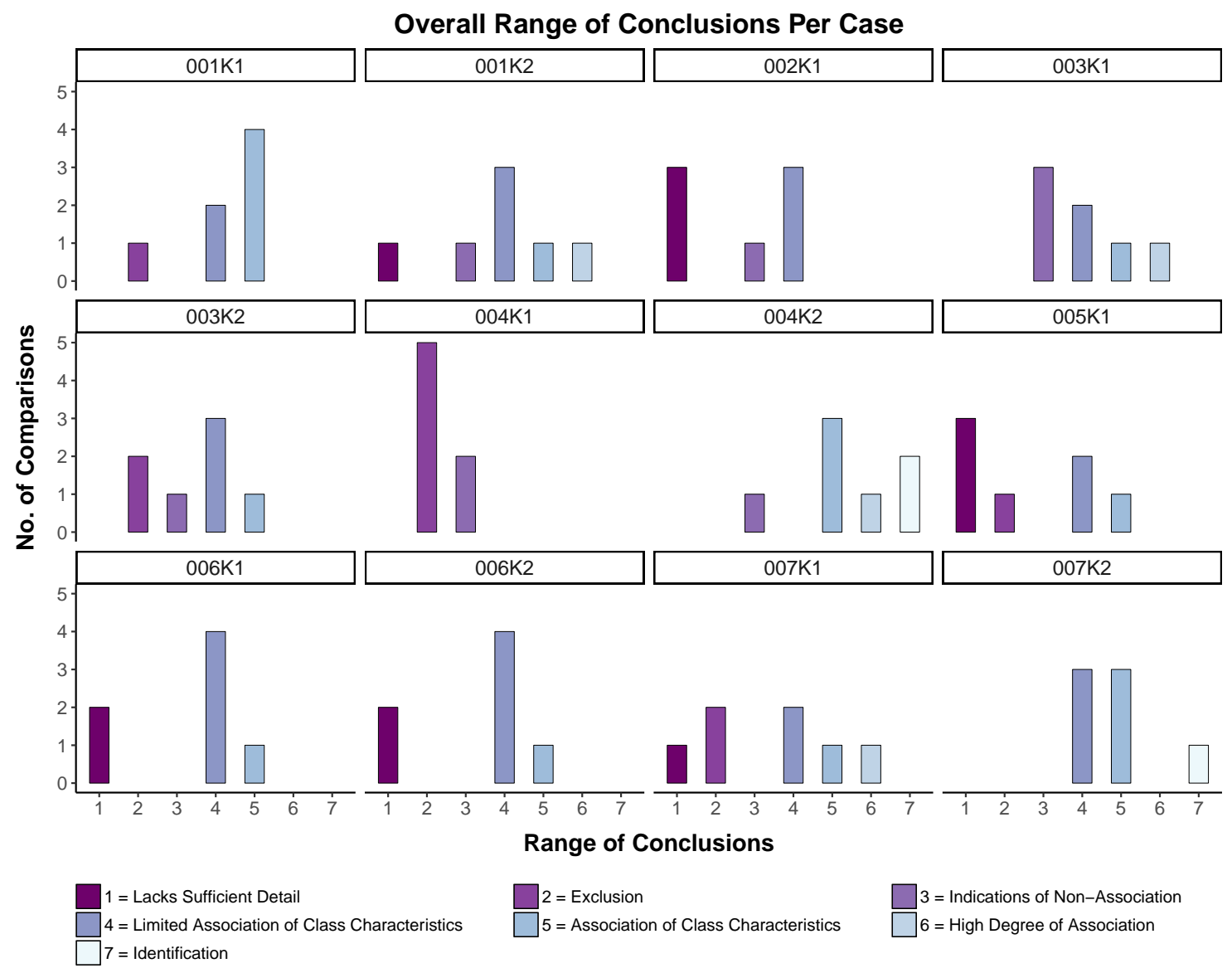

Figure 3.1: Overall range of conclusions for each questioned-to-known comparison. 
In Figure 3.2, for known matches, participants most of the time preferred to report a conclusion of "limited association of class characteristics" (19\%), followed by "association of class characteristics" (11\%) and finally "lacks sufficient detail" (7\%). However, for known non-matches, participants were more likely to favor "limited association of class characteristics" (14\%), followed by "exclusion" (11\%), and then "association of class characteristics" (10\%). In both cases, the decision maker may be more comfortable or confident in leaning toward the negative end of the association scale (26\% of comparisons for known matches and $31 \%$ of comparisons for known non-matches), as opposed to making a positive association decision on the case (16\% of comparisons for known matches and $12 \%$ of comparisons for known non-matches). This tendency to lean toward dissociating or excluding the questioned impression from the exemplar even for known matches may be due to lack of discipline-specific training, education, and experience in forensic footwear examination, thus resulting in difficulty interpreting the substrate-media interaction or the characteristics found in the questioned impression.

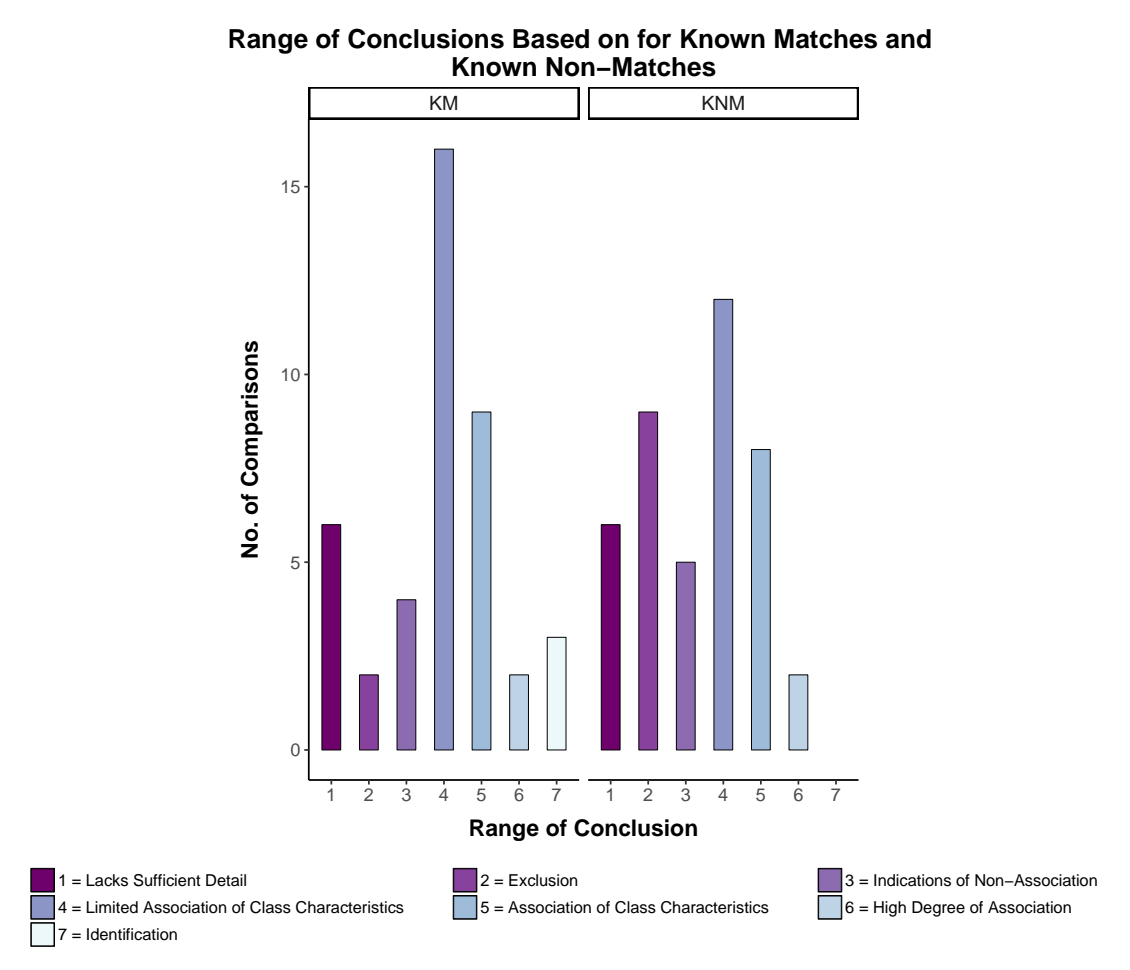

Figure 3.2: Distribution of conclusions for known matches and known non-matches.

Information entropy is the average (expected) amount of information in a certain event (Equation (3.1)), where $n$ denotes the number of occurrences and $p_{i}$ is the probability 
of obtaining the decision value 15]. Entropy is commonly expressed as bits, where the logarithm is of base 2 15. The lowest possible value for entropy is 0 , in which all cases were evaluated to the same decision, whereas the maximum entropy possible depends on the number of elements within the set, given by Equation (3.2) 15. Maximum entropy is obtained if each decision attribute has an equal probability of occurring. High entropy means that there are many possible outcomes with small probabilities such that when a case is randomly selected, one would expect a different decision than previous cases [15]. This also indicates high unpredictability or greater disorder, which means more information will be gained from this set of cases.

$$
\begin{array}{r}
\operatorname{Entropy}(S)=\sum_{i=1}^{n}-p_{i} \log _{2}\left(p_{i}\right) \\
S_{\text {max }}=\log _{2}(n)
\end{array}
$$

For each comparison, as well as known match/non-match category, the entropy was calculated to ascertain the distribution of the decision attributes within the set, as shown in Table 3.1. Relative to the maximum entropy and compared to the other cases, the entropy for case 007K1 is the highest. For this case, the examiners were equally likely to exclude the known or conclude with "limited association of class characteristics." Conversely, case 004K1 has the lowest entropy, where the examiners were more likely to exclude the known exemplar than to conclude with any other decision.

Table 3.1: Entropy and maximum entropy values for each case, as well as known match (KM) and known non-match (KNM) categories. $S$ denotes entropy, and $S_{\max }$ denotes maximum entropy.

\begin{tabular}{lcc}
\hline Instance & Entropy $(S)$ & Max Entropy $\left(S_{\max }\right)$ \\
\hline $001 \mathrm{~K} 1$ & 1.378783 & \\
$001 \mathrm{~K} 2$ & 2.128085 & \\
$002 \mathrm{~K} 1$ & 1.448816 & \\
$003 \mathrm{~K} 1$ & 1.842371 & \\
$003 \mathrm{~K} 2$ & 1.842371 & \\
$004 \mathrm{~K} 1$ & 0.863121 & \multirow{2}{*}{2.807355} \\
$004 \mathrm{~K} 2$ & 1.842371 & \\
$005 \mathrm{~K} 1$ & 1.842371 & \\
$006 \mathrm{~K} 1$ & 1.378783 & \\
$006 \mathrm{~K} 2$ & 1.378783 & \\
$007 \mathrm{~K} 1$ & 2.235926 & \\
$007 \mathrm{~K} 2$ & 1.448816 & \\
\hline $\mathrm{KM}$ & 2.421032 & \\
$\mathrm{KNM}$ & 2.424025 & 5.392317 \\
\hline
\end{tabular}

Table 3.2 shows the acceptable range of conclusions for each case questioned impression- 
known exemplar (Q-K) comparison. Very few participants concluded each comparison within the acceptable range, with the exceptions of 004Q-004K1 and 006Q-006K1, in which $71 \%$ of the participants concluded within the accepted range. In contrast, all participants reported conclusions that were out of range for 001Q-001K2 (ground truth = identification) and 006Q-006K2 (ground truth = exclusion). Participants were shown to prefer more conservative conclusions, which suggests some internal compensation process at play.

Table 3.2: Number of participants (percentage in parentheses) reaching each conclusion for each case Q-K comparison. Note: Conclusions are denoted as LSD = lacks sufficient detail, $\mathrm{E}=$ exclusion, INA = indications of non-association, LA = limited association, $\mathrm{A}=$ association of class, HDA = high degree of association, ID = identification, and $\mathrm{PR}=$ practitioner partner conclusion. Highlighted cells represent the acceptable range of conclusions for each comparison.

\begin{tabular}{|c|c|c|c|c|c|c|c|c|}
\hline \multirow{2}{*}{ Case Q-K Comparison } & \multicolumn{8}{|c|}{ Conclusions } \\
\hline & LSD & $\mathrm{E}$ & INA & LA & & HDA & ID & PR \\
\hline 001Q - 001K1 & & $1(14)$ & & $2(29)$ & $4(57)$ & & & $\mathrm{E}$ \\
\hline $001 \mathrm{Q}-001 \mathrm{~K} 2$ & $1(14)$ & $1(14)$ & $3(43)$ & $1(14)$ & $1(14)$ & & & ID \\
\hline $002 \mathrm{Q}-002 \mathrm{~K} 1$ & $3(43)$ & & $1(14)$ & $3(43)$ & & & & $\mathrm{A}$ \\
\hline $003 \mathrm{Q}-003 \mathrm{~K} 1$ & & & $3(43)$ & $2(29)$ & $1(14)$ & $1(14)$ & & A/HDA \\
\hline $003 \mathrm{Q}-003 \mathrm{~K} 1$ & & $2(29)$ & $1(14)$ & $3(43)$ & $1(14)$ & & & INA \\
\hline $004 \mathrm{Q}-004 \mathrm{~K} 1$ & & $5(71)$ & $2(29)$ & & & & & $\mathrm{E}$ \\
\hline $004 \mathrm{Q}-004 \mathrm{~K} 2$ & & & $1(14)$ & & $3(43)$ & $1(14)$ & $2(29)$ & ID \\
\hline $005 \mathrm{Q}-005 \mathrm{~K} 1$ & $3(43)$ & $1(14)$ & & $2(29)$ & $1(14)$ & & & $\mathrm{E}$ \\
\hline $006 \mathrm{Q}-006 \mathrm{~K} 1$ & $2(29)$ & & & $4(57)$ & $1(14)$ & & & $\mathrm{A}$ \\
\hline $006 \mathrm{Q}-006 \mathrm{~K} 2$ & $2(29)$ & & & $4(57)$ & $1(14)$ & & & $\mathrm{E}$ \\
\hline $007 \mathrm{Q}-007 \mathrm{~K} 1$ & $1(14)$ & $2(29)$ & & $2(29)$ & $1(14)$ & $1(14)$ & & $\mathrm{E}$ \\
\hline $007 \mathrm{Q}-007 \mathrm{~K} 2$ & & & & $3(43)$ & $3(43)$ & & $1(14)$ & ID \\
\hline
\end{tabular}

\subsection{Decision Rules}

In this section, the dominance-based rough set analysis is discussed with regard to three aspects: decision class approximations, rule generation, and significance of induced rules based on the conditional attributes used.

The overall decision table was analyzed using the dominance-based rough set approach. Using this analysis, information regarding each participant's preference order of the criteria, as well as similarity with respect to qualitative and quantitative evaluations, were taken into account. There are seven class unions based on the SWGTREAD scale, starting with "at most identification" $\left(C l_{7}^{\geq}\right)$, "at least high degree of association or more similar" $\left(C l_{6}^{\geq}\right)$, "at least association of class characteristics or more similar" $\left(C l_{5}^{\geq}\right)$, "at least limited association of class characteristics or more similar" $\left(\mathrm{Cl}_{4}^{\geq}\right)$, "at least indications of non-association or more similar" $\left(\mathrm{Cl}_{3}^{\geq}\right)$, "at least exclusion or more similar" $\left(\mathrm{Cl}_{2}^{\geq}\right)$, and "at most lacks sufficient detail" $\left(\mathrm{Cl}_{1}^{\leq}\right)$. The decision "at most identification" is the maximum degree of similarity and "at least exclusion" is the maximum degree of dissimilarity. Therefore, the inclusion of a particular case, $x$, in a lower approximation of a class union 
will have one of the following example interpretations:

- $x \in \underline{P}(C l \geqslant)$ means " $x$ certainly leads to at least identification"

- $x \in \underline{P}(C l \stackrel{\geq}{\square})$ means " $x$ certainly leads to at least high degree of association or more similar"

- $x \in \underline{P}(C l \leq 5)$ means " $x$ certainly leads to at most high degree of association or less similar"

- $x \in \underline{P}\left(C l_{1}^{\leq}\right)$means " $x$ certainly leads to at most lacks sufficient detail"

Conversely, if $x$ is included in an upper approximation of a class union, then one of the relevant following interpretations apply:

- $x \in \bar{P}\left(C l_{7}^{>}\right)$means " $x$ possibly leads to at least identification"

- $x \in \bar{P}\left(C l \geq \frac{\geq}{6}\right)$ means " $x$ possibly leads to at least high degree of association or more similar"

- $x \in \bar{P}(C l \leq)$ means " $x$ possibly leads to at most high degree of association or less similar"

- $x \in \bar{P}\left(C l_{1}^{\leq}\right)$means " $x$ possibly leads to at most lacks sufficient detail"

Using the attribute set in Table 2.4, the decision class unions were approximated to form the lower and upper approximations, $\underline{P}$ and $\bar{P}$, respectively. For all upward class unions, all approximated sets were "crisp," indicating that there were no ambiguous cases for any union (Table 3.3). Conversely, there were no cases found in the lower approximation sets for the downward class unions, which meant that all approximated sets were "rough" (Table 3.3).

Table 3.3: The table shows the number of "boundary" cases for each downward union. These boundary cases were found in the upper approximation sets of each downward union. Note that the figures provided do not exclude repeated occurences of the same case across various unions.

\begin{tabular}{|c|c|}
\hline Approximated sets & No. of cases in boundary regions \\
\hline$P\left(C l_{6}^{\frac{L}{6}}\right)$ & 81 \\
\hline$P\left(C l_{5}^{-}\right)$ & 77 \\
\hline$P\left(C l=\frac{<}{4}\right)$ & 60 \\
\hline$P\left(\mathrm{Cl}_{3}^{\frac{ \pm}{3}}\right)$ & 32 \\
\hline$P\left(C l_{2}^{\leq}\right)$ & 23 \\
\hline$P\left(C l_{1}^{-}\right)$ & 12 \\
\hline
\end{tabular}

For each upward class union, Table 3.4 shows the lower approximation sets and the number of cases that constitutes each set. There are no boundary cases for upward class 
unions, so rules that are eventually induced from these approximated sets are deemed to be "certain."

Table 3.4: Number of cases in each approximated set for upward class unions. There are no boundary cases. Note that the figures provided do not exclude repeated occurences of the same case across various unions.

\begin{tabular}{|c|c|}
\hline Approximated sets & Number of cases in set \\
\hline$P\left(C l_{7}^{>}\right)$ & 7 \\
\hline$P\left(C l_{6}^{\circ}\right)$ & 4 \\
\hline$P\left(C l \frac{P}{5}\right)$ & 24 \\
\hline$P\left(C l \frac{?}{4}\right)$ & 52 \\
\hline$P\left(C l \frac{>}{3}\right)$ & 61 \\
\hline$P\left(C l \frac{\$}{2}\right)$ & 72 \\
\hline
\end{tabular}

The approximation sets for both downward (Table 3.3) and upward (Table 3.4) class unions were then used to induce possible and certain rules for the respective class union.

Given the reduced set of attributes in Table 2.4, a total of 21 "minimum cover rules" were induced (i.e., the rule set does not contain redundant rules) based on the lower and upper approximations of class unions. There were 13 certain rules generated for the upward class union (Table 3.5), whereas for the downward unions, there were eight possible rules induced (Table 3.6).

For each rule, the decision, support, strength, certainty, and lift measures were also calculated. Support is a measure of the number of cases to which the rule is applicable within the dataset, and strength is the proportion of these cases given the total number of cases in the dataset, which is 84 examinations. The certainty factors indicate the proportion of cases in the dataset to which both the conditional and decision part of the rule are applicable, whereas the lift factors indicate the proportion of cases with the particular decision in the dataset to which the conditional part of the rule is applicable.

Of the 13 certain rules induced in Table 3.5, there was one rule that involved at least "indications of non-association" decisions or more similar, three rules each for cases with conclusions of at least "association of class characteristics" or more similar up to at most "identification." In this rule set, participants' educational background or practical experience was a factor commonly considered in the decision rules (nine out of 13 rules consisted of some form of experience as a condition), followed by participants' perceived value of the physical size of the design (four out of 13 rules consisted of this feature as a condition).

Using Rule 1 as an example, the rules and their respective measures in Table 3.5 can be interpreted as follows:

- If totality of the questioned impression is about $90 \%$ and there are about 2 features with value for identification, then the case will certainly be concluded with "identification." 
Table 3.5: Certain rules induced from the lower approximation of the upward class union. Each of the decision is represented by a numeral, where $3=$ "indications of nonassociation," $4=$ "limited association of class characteristics," $5=$ "association of class characteristics," $6=$ "high degree of association," and $7=$ "identification." Note: FS denotes forensic science, LCV is leucocrystal violet.

\begin{tabular}{|c|c|c|c|c|c|c|}
\hline No. & Conditions & Decision & Support & Strength & Certainty & Lift \\
\hline 1. & (Totality about $90 \%$ ) and (Features with value for identification about 2) & & & & & 0.33 \\
\hline 2. & (Experience in FS $\geq 6-8$ years) and (Enhancement $=$ Digital $)$ & $\mathrm{D} \geq 7$ & 1 & 0.01 & 0.16 & 0.33 \\
\hline 3. & (Enhancement $=$ Magnetic powder $)$ and (Physical size of design $=$ Value for association $)$ & $\mathrm{D} \geq 7$ & 1 & 0.01 & 0.09 & 0.33 \\
\hline 4. & (Clarity $\geq$ High) and (Experience in FS $\geq 6-8$ years) & $\mathrm{D} \geq 6$ & 3 & 0.03 & 0.25 & 0.43 \\
\hline 5. & (Internship focus on pattern sciences $=\overline{\text { Yes}}$ ) and (Totality about $50 \%)$ & $\mathrm{D} \geq 6$ & 3 & 0.03 & 0.17 & 0.43 \\
\hline 6. & (Internship focus on pattern sciences $=$ Yes) and (Internship site has pattern analysis services $=$ Yes) & $D \geq 6$ & 6 & 0.07 & 0.17 & 0.86 \\
\hline 7. & (Internship focus on pattern sciences $=$ Yes) and (Totality about $70 \%)$ & $\mathrm{D} \geq 5$ & 5 & 0.05 & 0.25 & 0.21 \\
\hline 8. & (Physical size of design $=$ Value for association $)$ and (Physical size of outsole $=$ Value for association $)$ & $\mathrm{D} \geq 5$ & 16 & 0.19 & 0.14 & 0.67 \\
\hline 9. & (Physical size of design $=$ Value for association) and (Examined/Compared pattern evidence in coursework $\geq$ Rarely) & $\mathrm{D} \geq 5$ & 22 & 0.26 & 0.39 & 0.92 \\
\hline 10. & (Clarity $\geq$ Moderate) and (Medium $=$ Dust) & $D \geq 4$ & 2 & 0.02 & 0.33 & 0.03 \\
\hline 11. & (Outsole design $=$ Value for association) and (Experience in $\mathrm{FS} \geq 1-2$ years) & $D \geq 4$ & 46 & 0.55 & 0.64 & 0.88 \\
\hline 12. & (Clarity $\geq$ Low) and (Experience in FS $\geq 1-2$ years) & $\mathrm{D} \geq 4$ & 52 & 0.62 & 0.62 & 1.00 \\
\hline 13. & (Experience in $\mathrm{FS} \geq 3-5$ years) and (Enhancement $=\mathrm{LCV}$ ) & $\mathrm{D} \geq 3$ & 29 & 0.34 & 0.81 & 0.48 \\
\hline
\end{tabular}

- Rule 1 has only 1 case supporting this implication.

- Relative to the dataset of 84 comparisons, Rule 1 has about $1 \%$ coverage or strength.

- For certainty, if a case had a questioned impression that retained about $90 \%$ of the footwear and the examiner found about 2 features with value for identification, then there is $100 \%$ certainty that the case will be concluded as "identification."

- For lift, if a case has conclusion of "identification," then there is $33 \%$ probability that the questioned impression retained about $90 \%$ of the footwear and the examiner found about 2 features with value for identification.

In Table 3.5. 75\% of the rules indicated that the participant's background may have some correlation with level of confidence associated with their decision-making. Rules 2 and 4-7 indicated that long-term experience within the forensic science field and performing practical tasks in a pattern analysis subfield could help the participant to assess the case and reach a more confident decision (i.e., "high degree of association" and "identification"). This is further supported by Rules 11 and 12, with the exception of Rule 9, which show that participants with some experience in pattern analysis, or more, are able to select a less ambiguous conclusion category from the scale as they attempt to apply their understanding of the SWGTREAD guidelines.

As for case features, it can be inferred from Rule 1 that participants can discern associative details between the impression and exemplar, given that the totality of the impression is about $90 \%$ or the clarity is at least high. Rules 5-12 also suggest that participants use a variety of characteristics, and particularly rely on class characteristics more than subclass or randomly acquired characteristics in determining mid-range conclusions, which suggests reasonable adherence to SWGTREAD guidelines.

Table 3.6 shows a total of 10 possible rules obtained from the upper approximation of downward class unions. About $40 \%$ of the rules involved at most "lacks sufficient detail" decisions and $30 \%$ involved at most "exclusion" conclusions, with remaining rules 
ranging from at most "indications of non-association" to at most "association of class characteristics" or less similar.

Table 3.6: Possible rules generated from the upper approximation of the downward unions, as well as the quality measures of the rules. Each of the decision is represented by a numeral, where $1=$ "lacks sufficient detail," $2=$ "exclusion," $3=$ "indications of nonassociation," $4=$ "limited association of class characteristics," and $5=$ "association of class characteristics." Note: FS denotes forensic science.

\begin{tabular}{|c|c|c|c|c|c|c|}
\hline No. & Conditions & Decision & Support & Strength & Certainty & Lift \\
\hline 1. & $($ Medium $=$ Dust $)$ and $($ Outsole design $=$ Value for exclusion $)$ & $\mathrm{D} \leq 1$ & & & & 0.25 \\
\hline 2. & $($ Substrate $=$ Vinyl tile $)$ and $($ Medium $=$ Dust $)$ & $\mathrm{D} \leq 1$ & 6 & 0.07 & 0.43 & 0.50 \\
\hline 3. & $($ Medium $=$ Dust $)$ and $($ Enhancement $=$ Digital $)$ & $\mathrm{D} \leq 1$ & 10 & 0.12 & 0.36 & 0.83 \\
\hline 4. & (Totality about $55 \%$ ) and (Substrate $=$ Ceramic tile) & $\mathrm{D} \leq 1$ & & 0.02 & 0.11 & 0.17 \\
\hline 5. & $($ Medium $=$ Blood $)$ and $($ Physical size of outsole design $=$ Value for a & $\mathrm{D} \leq 2$ & 3 & 0.03 & 0.14 & 0.13 \\
\hline 6. & (Outsole design = Value for association) and (Examined/Compared pattern evidence in course & $\mathrm{D} \leq 2$ & 18 & 0.21 & 0.25 & 0.78 \\
\hline 7. & (Clarity $\leq$ High) and (Experience in FS $\leq 6-8$ years) & $\mathrm{D} \leq 2$ & 23 & 0.27 & 0.27 & 1.00 \\
\hline 8. & (Physical size of design $=$ Value for association) and (Education concentration $\mathrm{i}$ & $\mathrm{D} \leq 3$ & 21 & 0.25 & 0.32 & 0.66 \\
\hline 9. & (Physical size of design $=$ Value for association) and (Clarity $\leq$ Moderat & $\mathrm{D} \leq 4$ & 31 & 0.37 & 0.60 & 0.52 \\
\hline 10. & $($ Medium $=$ Wax $)$ and (Internship site has pattern analysis services $=$ Yes $)$ & $\mathrm{D} \leq 5$ & 5 & 0.06 & 0.63 & 0.06 \\
\hline
\end{tabular}

Possible rules and their respective measures in Table 3.6 can be interpreted as follows, using Rule 1 as an example:

- If medium is dust and the examiner evaluated the outsole design to have value for exclusion, then the case will possibly be concluded with "lacks sufficient detail."

- Rule 1 has 3 cases supporting this implication.

- Relative to the dataset of 84 comparisons, Rule 1 has about 3\% coverage or strength.

- For certainty, if a case had a questioned impression deposited in dust and the examiner found that the outsole design had value for exclusion, then there is $100 \%$ certainty that the case will be concluded as "lacks sufficient detail."

- For lift, if a case has conclusion of "lacks sufficient detail," then there is $25 \%$ probability that the questioned impression was deposited in dust and the examiner found the outsole design to have value for exclusion.

Based on Table 3.6, regardless of participants' background or familiarity with pattern analysis, media type influenced their decisions more frequently than the other factors $(50 \%$ of the rules involved medium as a condition). Cases in which dust was the medium were more likely to be assigned a less confident decision (Rules 1-3). As for cases with blood as medium and ceramic tile for substrate (Rules 4 and 5), participants seemed to either exclude the exemplar or conclude with "lacks sufficient detail." This suggests that some participants may have had some difficulty interpreting the medium-substrate interaction, and therefore were unable to assign a conclusion confidently. Rules 6 and 7 served to highlight the diversity of participants' breadth of experience and their wide-ranging abilities to perceive details in footwear impression, as they may rely on factors other than outsole design to determine an exclusion. Rule 8 suggests that while participants may have had some educational background in pattern sciences and were at least able to determine the 
value of the physical size of the design, some may still be uncertain about excluding the exemplar completely, which led to a conclusion of "indications of non-association." This rule also implies that participants, given their background in pattern analysis, were at most comfortable with exclusion-type decisions when uncertain. Rule 9 indicates that participants were able to associate the exemplar to the impression in a limited capacity (based on physical size of design), if the clarity of the questioned impression was perceived to be at most moderate. Interestingly, based on Rule 10, not many participants assigned a more confident conclusion (e.g., "high degree of association"), and even participants who interned at sites offering pattern analysis services may find some difficulty with interpreting wax-based questioned impression.

Overall, the rules induced in Tables 3.5 and 3.6 suggest that without domain-specific knowledge, training or experience, decision makers may not be able to arrive at the appropriate conclusions or make decisions confidently. This is supported by the fact that most of the decisions made to associate a questioned impression and known footwear lie in the "ambiguous" areas of the SWGTREAD scale (i.e., "limited association of class characteristics" and "association of class characteristics"), and when unable to arrive at a definite conclusion, participants were more likely to conclude "lacks sufficient detail" than to completely exclude the known footwear.

The reduced set of attributes in Table 2.4 was further minimized to exclude all conditions from the background survey, as well as the substrate and medium variables from case examination. With this reduced set, 24 decision rules were induced. The upward class unions yielded nine "certain" rules while the downward class unions yielded 13 "possible" rules.

Table 3.7: Certain rules induced from the lower approximation of the upward class union. Each of the decision is represented by a numeral, where $3=$ "indications of nonassociation," $4=$ "limited association of class characteristics," $5=$ "association of class characteristics," $6=$ "high degree of association," and $7=$ "identification." Note: FS denotes forensic science, LCV is leucocrystal violet.

\begin{tabular}{|c|c|c|c|c|c|c|}
\hline No. & Conditions & Decision & Support & Strength & Certainty & Lift \\
\hline 1. & (Totality about $90 \%$ ) and (Features with value for identification about 2) & $\mathrm{D} \geq 7$ & 1 & 0.01 & 1.00 & 0.33 \\
\hline 2. & (Outsole design $=$ Value for association) and (Totality about 30\%) & $\mathrm{D} \geq 7$ & 1 & 0.01 & 0.14 & 0.33 \\
\hline 3. & (Features with value for limited association about 1 ) and (Physical size of outsole $=$ Value for association) & $\mathrm{D} \geq 7$ & 1 & 0.01 & 0.09 & 0.33 \\
\hline 4. & Totality about 50\%) and (Physical size of outsole = Insufficient detail) & $\mathrm{D} \geq 6$ & 1 & 0.01 & 0.10 & 0.14 \\
\hline 5 . & (Totality about $75 \%$ ) and (Physical size of outsole $=$ Value for association) & $\mathrm{D} \geq 6$ & 2 & 0.02 & 0.11 & 0.29 \\
\hline 6. & (Totality about $73-80 \%$ ) & $\mathrm{D} \geq 6$ & 4 & 0.05 & 0.08 & 0.57 \\
\hline 7. & (Clarity $\geq$ High) and (Features with value for limited association about 4 ) & $\mathrm{D} \geq 5$ & 1 & 0.01 & 0.20 & 0.04 \\
\hline 8. & (Clarity $\geq$ Moderate $)$ and $($ Outsole design $=$ Value for exclusion $)$ & $\mathrm{D} \geq 4$ & 3 & 0.04 & 0.60 & 0.06 \\
\hline 9 . & (Clarity $\geq$ Moderate) and (Features with value for exclusion about 2 ) & $\mathrm{D} \geq 3$ & 1 & 0.01 & 0.25 & 0.02 \\
\hline
\end{tabular}

The rules from Table 3.7 suggest that there may be other underlying factors involved in interpreting the totality, clarity as well as the value of the class characteristics present in the questioned impression. This can be seen in Rules 2, 4 and 5, where Rule 2 seems to contradict Rule 1, as the participant seemed to be using fewer features to determine "identification" for the particular case. There may have been participants who were capable of doing so, but their backgrounds were not considered in the attribute set and in general a conclusion of "identification" can only be reached when RACs are present. 
Table 3.8: Possible rules generated from the upper approximation of the downward unions, as well as the quality measures of the rules. Each of the decision is represented by a numeral, where 1 = "lacks sufficient detail," $2=$ "exclusion," $3=$ "indications of nonassociation," $4=$ "limited association of class characteristics," and $5=$ "association of class characteristics." Note: FS denotes forensic science.

\begin{tabular}{|c|c|c|c|c|c|c|}
\hline No. & Conditions & Decision & Support & Strength & Certainty & Lift \\
\hline 1. & (Outsole design $=$ Insufficient detail) and (Totality about $20 \%)$ & $\mathrm{D} \leq 1$ & & & & 0.17 \\
\hline 2. & (Physical size of outsole $=$ Insufficient detail) and (Physical size of design $=$ Insufficient detail & $\mathrm{D}<\overline{1}$ & 2 & 0.02 & 1.00 & 0.17 \\
\hline 3. & Features with value for indications of non-association about 1 ) and (Outsole design = Insufficient detail) & $\mathrm{D} \dot{\mathrm{i}} 1$ & 1 & 0.01 & 0.50 & 0.08 \\
\hline 4. & (Physical size of outsole = Value for exclusion) and (Features with value for association about 1) & $\mathrm{D} \overline{\leq} 1$ & 2 & 0.02 & 0.33 & 0.17 \\
\hline 5. & (Physical size of outsole $=$ Value for exclusion) and (Outsole design = Value for association) & $\mathrm{D} \leq 1$ & 3 & 0.04 & 0.30 & 0.25 \\
\hline 6. & (Totality about $42 \%$ ) and (Physical size of outsole = Insufficient detail) & $\mathrm{D} \dot{\leq} 1$ & 1 & 0.01 & 0.13 & 0.08 \\
\hline 7. & $($ Physical size of outsole $=$ Value for association $)$ and (Totality about $50 \%)$ & $\mathrm{D}<\overline{1}$ & 2 & 0.02 & 0.11 & 0.17 \\
\hline & design $=$ Value for associatiol & $\mathrm{D}$ & 2 & 0.02 & 0.04 & 0.17 \\
\hline 9. & (Physical size of design $=$ Value for association $)$ and (Outsole design $=$ Value for exclusion $)$ & $\mathrm{D} \overline{\leq} 2$ & 1 & 0.01 & 0.20 & 0.04 \\
\hline 10. & $($ Physical size of design $=$ Value for association) and (Totality about $55 \%)$ & $\mathrm{D} \leq 3$ & 9 & 0.11 & 0.31 & 0.28 \\
\hline 11. & (Physical size of design $=$ Value for association) and (Clarity $\leq$ Moderate $)$ & $\mathrm{D} \leq 4$ & 31 & 0.37 & 0.60 & 0.52 \\
\hline 12. & Outsole design $=$ Value for association) and (Features with value for identification about 1 ) & $\mathrm{D} \leq 5$ & 2 & 0.02 & 0.50 & 0.03 \\
\hline 13. & (Physical size of outsole $=$ Not evaluated) and (Clarity $\leq$ Moderate) & $\mathrm{D} \dot{\leq} 6$ & 1 & 0.01 & 1.00 & 0.01 \\
\hline
\end{tabular}

Table 3.8 shows that participants were more inclined to use class characteristics rather than RACs or other specific characteristics in order to determine the case conclusion. Rules 2-8 demonstrate the participants' reluctance to conclude with "exclusion" even when they deem some class characteristics to be value for exclusion.

\subsection{Conclusion}

The present study demonstrates that novices lacking experience or those unfamiliar with standard practice in forensic footwear examination are more likely to interpret the criteria outlined in the SWGTREAD scale somewhat conservatively when assigning a conclusion for a case. However, having some experience in other pattern disciplines may yield skills that are transferrable to footwear comparison. While there are general trends showing that totality and clarity of the crime scene impression influences the examiner's interpretation of the features present, there is some evidence to support that the examiner's decisionmaking process does involve recalling or applying principles and practices obtained through their experience in pattern analysis.

\section{Bibliography}

[1] Burch AM, Durose MR, Walsh KA. Census of publicly funded forensic crime laboratories, 2009. vol. 24. Bureau of Justice Statistics; 2012. 
[2] Presidents Council of Advisors on Science and Technology (PCAST). Forensic Science in Criminal Courts: Ensuring Scientific Validity of Feature-Comparison Methods; 2016 (Accessed March 2017). Available from: https://obamawhitehouse.archives.gov/sites/default/files/ microsites/ostp/PCAST/pcast_forensic_science_report_final.pdf.

[3] Majamaa H, Ytti A. Survey of the conclusions drawn of similar footwear cases in various crime laboratories. Forensic Science International. 1996;82(1):109 120. European Meeting for Shoeprint and Toolmark Examiners. Available from: http://www.sciencedirect.com/science/article/pii/037907389601972X.

[4] Hammer L, Duffy K, Fraser J, Nic Daéid N. A study of the variability in footwear impression comparison conclusions. Journal of Forensic Identification. 2013;63(2):205-218.

[5] Scientific Working Group for Shoeprint and Tire Tread Evidence (SWGTREAD). Standard Terminology for Expressing Conclusions of Forensic Footwear and Tire Impression Examinations; 2006 (Accessed January 2017). Available from: http://treadforensics.com/images/swgtread/standards/archived/ swgtread_10_terminology_conclusions_200603_201302.pdf. Available from: http://treadforensics.com/images/swgtread/standards/archived/ swgtread_10_terminology_conclusions_200603_201302.pdf

[6] Scientific Working Group for Shoeprint and Tire Tread Evidence (SWGTREAD). Range of Conclusions Standard for Footwear and Tire Impression Examinations; 2013 (Accessed December 2016). Available from: https://www.swgtread.org/ images/documents/standards/published/swgtread_10_conclusions_range_ 201303.pdf. Available from: https://www.swgtread.org/images/documents/ standards/published/swgtread_10_conclusions_range_201303.pdf.

[7] Ulery BT, Hicklin RA, Buscaglia J, Roberts MA. Accuracy and reliability of forensic latent fingerprint decisions. Proceedings of the National Academy of Sciences. 2011;108(19):7733-7738. Available from: http://www.pnas.org/content/108/ 19/7733.abstract

[8] Sikder IU, Munakata T. Application of rough set and decision tree for characterization of premonitory factors of low seismic activity. Expert Systems with Applications. 2009;36(1):102-110. Available from: http://www.sciencedirect.com/science/ article/pii/S0957417407004460.

[9] Pawlak Z. Rough sets. International Journal of Computer \& Information Sciences. 1982;11(5):341-356.

[10] Pawlak Z. Rough sets and intelligent data analysis. Information Sciences. 2002;147(14):1-12. Available from: http://www.sciencedirect.com/science/article/ pii/S0020025502001974.

[11] Liou JJH. A novel decision rules approach for customer relationship management of 
the airline market. Expert Systems with Applications. 2009;36(3, Part 1):43744381. Available from: http://www.sciencedirect.com/science/article/pii/ S0957417408002443.

[12] Greco S, Matarazzo B, Słowiński R. Rough sets methodology for sorting problems in presence of multiple attributes and criteria. European Journal of Operational Research. 2002;138(2):247-259.

[13] SWGTREAD. Standard for Terminology Used for Forensic Footwear and Tire Impression Evidence; 2013. Available from: http://www.swgtread.org/images/ documents/standards/published/swgtread_15_terminology_evidence _ 201303.pdf.

[14] Greco S, Matarazzo B, Słowiński R, Stefanowski J. Variable consistency model of dominance-based rough sets approach. In: Rough Sets and Current Trends in Computing. Springer; 2001. p. 170-181.

[15] Gray RM. Entropy and Information Theory. Springer-Verlag; 1990. 


\section{A. SWGTREAD Conclusion Scale}

\section{SWGTREA}

\section{Range of Conclusions Standard for Footwear and Tire Impression Examinations (03/2013)}

\section{Scope}

1.1 This standard is provided to define the range of conclusions applicable to forensic footwear and tire impression examinations.

1.2 The range of conclusions in this standard may not address every variable in every examination. Wording expressing conclusions in each case should be constructed specific to the results of the examination in that case.

1.3 This standard is not a substitute for training in the examination of forensic footwear and tire impression evidence. Completion of a training program and experience are essential to understanding and applying the principles outlined in this standard.

1.4 This standard is not intended to provide a specific format for writing an expert report. Refer to Standard for Report Writing for Footwear and Tire Impression Examinations for examples of wording for conclusions (under construction).

1.5 This standard is not intended to include the examination process. Refer to Standard for the Examination of Footwear and Tire Impression Evidence for further guidance.

\section{Terminology}

Refer to the Standard for Terminology Used for Forensic Footwear and Tire Impression Evidence for a definition of terms used in this document.

\section{Significance and Use}

3.1 The purpose of this document is to standardize the range of conclusions for footwear and tire impression evidence examinations.

3.2 The range of conclusions regarding footwear and tire impression evidence should be readily understandable and transparent. 


\section{Opinions and Conclusions}

The following descriptions are meant to provide context to the levels of opinions reached in footwear and tire impression comparisons. Each level may not include every variable in every case. This applies to both partial and full impressions.

\subsection{Lacks sufficient detail}

4.1.1 No comparison was conducted: the examiner determined there were no discernible questioned footwear/tire impressions or features present. This opinion applies when there is insufficient detail to conduct any comparison.

In the opinion of the examiner, an impression was either not present or the impression lacked sufficient detail for any comparison.

4.1.2 A comparison was conducted: the examiner determined that there was insufficient detail in the questioned impression for a meaningful conclusion. This opinion only applies to the known footwear or tire that was examined and does not necessarily preclude future examinations with other known footwear or tires.

In the opinion of the examiner, the impression lacked sufficient detail for a meaningful conclusion regarding the particular known footwear outsole or tire tread.

4.2 Exclusion - This is the highest degree of non-association expressed in footwear and tire impression examinations. Sufficient differences were noted in the comparison of class and/or randomly acquired characteristics between the questioned impression and the known footwear or tire.

In the opinion of the examiner, the particular known footwear or tire was not the source of, and did not make, the impression.

4.3 Indications of non-association - The questioned impression exhibits dissimilarities when compared to the known footwear or tire; however, the details or features were not sufficiently clear to permit an exclusion.

In the opinion of the examiner, dissimilarities between the questioned impression and the known footwear or tire indicated non-association; however, the details or features were not sufficient to permit an exclusion.

4.4 Limited association of class characteristics - Some similar class characteristics were present; however, there were significant limiting factors in the questioned impression that did not permit a stronger association between the questioned impression and the known footwear or tire. These factors may include but were not limited to: insufficient detail, lack of scale, improper position of scale, improper photographic techniques, distortion or significant lengths of time between the date of the occurrence and when the footwear or tires were recovered that could 
account for a different degree of general wear. No confirmable differences were observed that could exclude the footwear or tire.

In the opinion of the examiner, factors (such as those listed above) have limited the conclusion to a general association of some class characteristics. Other footwear or tires with the same class characteristics observed in the impression are included in the population of possible sources.

4.5 Association of class characteristics - The class characteristics of both design and physical size must correspond between the questioned impression and the known footwear or tire. Correspondence of general wear may also be present.

In the opinion of the examiner, the known footwear or tire is a possible source of the questioned impression and therefore could have produced the impression. Other footwear or tires with the same class characteristics observed in the impression are included in the population of possible sources.

4.6 High degree of association - The questioned impression and known footwear or tire must correspond in the class characteristics of design, physical size, and general wear. For this degree of association there must also exist: (1) wear that, by virtue of its specific location, degree and orientation make it unusual and/or (2) one or more randomly acquired characteristics.

In the opinion of the examiner, the characteristics observed exhibit strong associations between the questioned impression and known footwear or tire; however, the quality and/or quantity were insufficient for an identification. Other footwear or tires with the same class characteristics observed in the impression are included in the population of possible sources only if they display the same wear and/or randomly acquired characteristics observed in the questioned impression.

4.7 Identification - This is the highest degree of association expressed by a footwear and tire impression examiner. The questioned impression and the known footwear or tire share agreement of class and randomly acquired characteristics of sufficient quality and quantity.

In the opinion of the examiner, the particular known footwear or tire was the source of, and made, the questioned impression. Another item of footwear or tire being the source of the impression is considered a practical impossibility.

\section{Limitations}

Accurate and reliable data and/or statistical models for use in calculations do not currently exist. Therefore, SWGTREAD does not support the use of statistics to determine the strength of conclusions related to shoe and tire impression evidence at this time. 


\section{Bibliography}

Abbott, J. R., Footwear Evidence, Charles C. Thomas Publisher: Springfield, IL, 1964.

Bodziak, W. J., Footwear Impression Evidence, 2nd ed., CRC Press: Boca Raton, FL, 2000.

Bodziak, W. J., Tire Tread and Tire Track Evidence, CRC Press: Boca Raton, FL, 2008.

Cassidy, Michael J., Footwear Identification, Canadian Government Publishing Center, 1980.

ENFSI EWG Marks, "Conclusion Scale for Shoeprint and Toolmarks Examinations", JFI, 56 (2), 2006.

Nause, Lawren, Forensic Tire Impression Identification; Canadian Police Research Centre, 2001. 


\section{B. SWGTREAD Footwear Terminology}

\section{SWGTREA}

\section{Standard for Terminology Used for Forensic Footwear and Tire Impression Evidence (03/2013)}

\section{Scope}

1.1 This Standard provides terminology that is commonly used in the forensic recovery and examination of footwear and tire impression evidence.

Note: There may be terms not in this Standard that appear in other sources.

\section{Terminology}

Accidental characteristic: See Randomly acquired characteristic.

Adhesive lifter: Any material coated with a tacky substance for the purpose of lifting footwear or fingerprint impressions.

Air bubble: A globule of air trapped within a solid material such as a footwear sole.

Aspect ratio: The proportion of the tire's height to its width.

Asymmetric tread design: A tire tread pattern where when a tread design is divided circumferentially, one half of the tread design is not a mirror image of the other half.

Bead: A hoop of steel wires that hold the tire on the rim.

Bias tire: A tire that has plies which cross over one another at an angle.

Bias-belted tire: A bias tire with added reinforced belts that lie beneath the tread.

BIO-FOAMR: A commercial product comprised of collapsible foam used primarily for the recording of anatomical impressions of a foot, and sometimes impressions of footwear soles.

Biscuit: Pre-formed or extruded pieces of soling compound that are placed in molds and pressed into the shape of a footwear sole or heel.

Blade: Thin pieces of metal in footwear and tire molds that result in molded sipes.

Blocker: An oversized outsole made of one or more components that is later cut to size. 
Blunt force pattern injury: An injury to the skin by an object resulting in a pattern that may replicate the design of an object. (Also known as a contusion.)

Brannock Device ${ }^{\circledR}$ : The registered name of a foot-measuring device.

CAD/CAM: Computer-Aided Design/Computer-Aided Manufacture.

Calendering: A process where raw rubber passes between a series of large steel calender rollers. The final calendar roller impresses the sole design into the rubber that is later cut into soles. Calender rollers are also used to help prepare raw rubber for the production of rubber biscuits for the compression molding process.

Carcass: The portion of the tire that includes the liner, plies, belts, and beads which forms the foundation for the tread and sidewall.

Cast: The result of filling a three-dimensional impression with an appropriate material.

Casting material: Dental stone, sulfur, or other suitable materials specifically used to accurately recover three-dimensional impressions. Some casting materials are also successful for lifting two-dimensional impressions.

Center rib: A rib that runs circumferentially and is evenly centered within the tire tread design.

Chart board: A solid laminated board with a covering of white paper on at least one side (not foam core board) used to provide a firm and smooth backing when obtaining known tire impressions.

Chemical etching: A process wherein a textured pattern is applied to selective areas of a mold surface. The mold is later dipped in an acid bath that etches the pattern into the mold. A chemically etched pattern is unique to a specific mold.

Chemiluminescence: Luminescence due to a chemical reaction.

Class characteristic: A feature that is shared by two or more items of footwear or tires. The footwear outsole or tire tread design and the physical size features of a footwear outsole or tire tread are two common manufactured class characteristics. General wear of the outsole or tire tread is also a class characteristic. Agreement of class characteristics alone does not provide a basis for identification however they reduce the possible number of footwear or tires that could have made an impression.

Clicker: A hydraulic machine that forces a steel die through outsole and/or midsole materials in a cookie-cutter fashion.

Coaxial light: Illumination from the precise direction of the imaging lens, either through the lens or with a beam-splitter in front of the lens. 
Compression molding: A method for making outsoles where the outsole material is placed into an open mold, which is then closed and subjected to heat and pressure. Soles made with this process are referred to as "pressed soles".

Consistency: The percentage of water in the water-to-powder ratio of a gypsum product such as dental stone. In this ratio, the powder will always be 100 . For example, a dental stone having a water-to-powder ratio of $30 / 100$ has a consistency of 30 .

Cord: Fabrics placed under tension and covered with rubber. Used to form the plies of the tire.

Correspond: A word used to describe agreement of class and randomly acquired characteristics in the context that reflects the footwear or tire is capable of having produced a certain feature such as design, general wear, etc.

Degree of wear: The extent to which a footwear outsole or tire tread has been eroded. Examples of degree of wear range from a footwear outsole or tire tread that is in a new and unworn condition to those that have considerable wear. The degree of wear continues to change as a footwear outsole or tire tread is worn.

Dental stone: A gypsum product generally having a pound per square inch (psi) rating of 8,000 or higher, commonly used to cast footwear and tire impressions.

Design: The manufactured pattern of a footwear outsole or tire tread. Design is a class characteristic.

Design/Size relationship: The tendency for a footwear outsole or tire tread design to have either more design elements, or larger design elements, or both, as the footwear or tire size increases throughout the size range produced.

Die cut: Outsoles or other footwear components produced by forcing a sharpened steel die through pre-formed outsole material with the assistance of a clicker machine.

Difference: A characteristic or feature that is so strong and reliable that it, in itself, demonstrates that the particular known footwear or tire was not the source of and did not make the impression. Usually a difference will be a different class characteristic, such as the specific design or specific physical size of the design. Normal variations in the impression process, the absence of cuts in a questioned impression that appear on the footwear or tire, or the normal advancement of wear with time do not necessarily constitute a comparative difference. (A difference should not be confused with a Dissimilarity.)

Direct attach: A manufacturing process where the upper of the footwear is lowered onto a sole plate in a mold cavity and the midsole or outsole material is injected directly onto the upper. This term also applies to open pour polyurethane molding 
where the lasted footwear upper is lowered into a mold containing poured polyurethane and an outsole, directly attaching both to the upper.

Directional tread design: A tire tread pattern that is optimized to work best when rotating in one direction only.

Dissimilarity: When a characteristic has the appearance of being potentially different but lacks sufficient detail for confirmation.

Distortion: An unclear or inaccurate representation of the footwear or tire in an impression due to interference in the impression-making process or its subsequent retrieval.

DOT number: Department of Transportation serial number assigned to every tire sold in the United States which gives information regarding the manufacturer, size, and date of manufacture of the tire.

Dry casting: A casting method utilizing the layering of dry dental stone powder and misted water.

Dry origin impressions: Impressions formed under dry conditions such as dry dust and dry residue impressions.

Dual tire assembly: A pair of tires mounted side-by-side on a fixed wheel assembly.

Electrical discharge machine (EDM): A machine used to produce molds by electrically burning away the undesired metal portions.

Electrostatic detection apparatus (ESDA) or Electrostatic detection device: An instrument used primarily to detect indented writing on documents, which can also be used to detect footwear and tire tread impressions on paper items.

Electrostatic dust lifter: An instrument that utilizes an electrostatic charge as a means of transferring dry origin impressions from a substrate to a film.

Electrostatic lifting: The process of using an electrostatic charge to transfer dry origin impressions from the substrate to a film.

Electrostatic lifting device: An instrument that utilizes electrostatic charges as a means of transferring dry origin impressions from a surface to a film.

Element/Design Element: A single component (lug, herringbone, wave, circle, etc.) of a footwear sole distinguished by its shape that, by itself or with other design elements, comprises the tread design on that sole. See Tread block.

Elimination: See Exclusion. 
Elimination impressions and/or photographs: Impressions and/or photographs taken of footwear and tires from known sources (police officers, paramedics, and their vehicles, etc.) for the purpose of discerning them from the questioned crime scene impressions.

Enhancement: Improving the ability to visualize an impression through physical, photographic, digital or chemical means or through the use of alternate light sources.

Ethylene vinyl acetate (EVA): A soling compound often produced in an expanded form.

Examination quality photographs: High quality photographs taken with a scale specifically for use in the physical comparison of footwear and tire impressions with known footwear and tires.

Exclusion: An opinion by an examiner that the particular known footwear or tire was not the source of, and did not make, the impression. This is the highest degree of non-association expressed in footwear and tire impression examinations.

Exemplar: See Test impression.

Feathering: See Schallamach pattern.

Fixative: Substance that stabilizes blood prior to enhancement. Also refers to any product that will stabilize the substrate prior to casting.

Flash: Small amounts of rubber and footwear soling compounds that have seeped between mold components during the footwear and tire molding process.

Fluorescence: Luminescence that is caused by the absorption of radiation at one wavelength followed by nearly immediate re-radiation usually at a different wavelength and that ceases almost at once when the incident radiation stops.

Footwear: Any apparel worn on the foot, such as shoes, boots, sandals, etc.

Forensic light source: A filtered light source that may be fixed or tunable to a variety of spectral ranges.

Foxing/Foxing strip: A strip of rubber wrapped around the lower part of some footwear to cover the gap or seam between the upper and the outsole.

Full circumference tire impression: An impression of a tire that represents a full rotation of that tire under load and thus represents its entire tread surface.

Full impression: An impression that represents all, or nearly all, of the heel to toe portions of the outsole or the full width and circumference of the tire.

Gelatin lifter: Gelatin applied to a pliable backing that can be used to lift impressions 
General sole design: A very general category of footwear sole patterns, i.e. herringbone pattern, lugged sole pattern, wave pattern, plain soles, etc.

General wear: The condition (degree and position of wear) of the overall footwear outsole or tire tread, ranging from new to extremely worn, related to its degree of use. General wear is a class characteristic that may be used to include or exclude footwear or tires.

Grooves: The space or channels that separate the tread ribs and elements. Circumferential grooves run around the circumference of the tire. Transverse or lateral grooves, also known as slots, run across the tire tread design.

Holes: The result of erosion of a footwear outsole or tire tread that is so extreme that it results in removal of the outer layers of sole or tread materials, often resulting in irregular edges. These irregular edges are randomly acquired characteristics. Random holes due to punctures are also randomly acquired characteristics.

Identicator@: An inkless method of recording footwear impressions on white chemically treated paper.

Identification: An opinion by an examiner that the particular known footwear or tire was the source of, and made, the impression. This is the highest degree of association expressed in footwear and tire impression examinations.

Identifying characteristics: See Randomly acquired characteristics.

Impression: The product of direct physical contact of an item, such as a footwear or tire, resulting in the transfer and retention of characteristics of that item.

Individual characteristics: See Randomly acquired characteristics.

Injection molding: A manufacturing method where the sole and/or midsole is made by forcing material into a closed mold. Outsoles can be molded individually as unit soles or directly onto the footwear upper as direct attach soles.

Improper photographic technique: When one or more essential procedures is/are not followed resulting in a limited ability to conduct an accurate examination. Some examples are: out of focus images, improper scale position, lack of a scale, and improper lighting.

Improper position of scale: Photographs taken of impressions where the scale is not on the same plane as the bottom of the impression or is not parallel to the camera back, film plane, and/or the digital sensor.

Insole: A cushioned liner that occupies the inner surface of an item of footwear where the foot rests and is placed there for comfort or protection. The insole may or may not be removable. 
Insufficient detail: Features which fall short of allowing the confirmation of certain class or randomly acquired characteristics.

Known impression: See Test impression.

Known footwear or tire: An item of footwear or tire that is compared to a questioned footwear or tire impression.

Label (manufacturer's sizing label): A label placed on the tongue or other inside surface of the footwear that contains information including but not limited to the manufacturer's name, shoe size, country of manufacturer, style number, dating information, barcodes, etc.

Lack of scale: When photographs do not contain a ruler or other acceptable linear scale, essential for enlarging a photograph to its natural size.

Latent impression: An impression not readily visible to the naked eye.

Last: A form made of wood, metal, or synthetic material that approximates the size and shape of a foot. The upper of the footwear is stretched over the last and held in a specific shape and size throughout the manufacturing process. The size on the manufacturer's label is directly related to the size of the last.

Liner: A thin layer of butyl rubber compound that holds the air inside the tire.

Logo: A name, design, or pattern that is the trademark of the manufacturer that may appear on the footwear or on the outsole.

Low profile: A term describing a tire that has a low aspect ratio, thus a short sidewall.

Manufacturing defect: Unintended damage, defects or flaws in the footwear outsole or tire tread that occurs during manufacturing, which depending on their cause, could result in class or randomly acquired characteristics.

Manufacturing variable: Variations that occur during the manufacturing process that do not appear on all of the footwear/tires but may appear on more than one. Examples would be the precise positioning of foxing strips, the precise cutting of die cut or Wellman cut soles, the positioning of stitching that is added to the bottom of some soles, or a bent sipe blade in a tire mold, etc.

Mikrosil ${ }^{\mathrm{TM}}$ : Silicone casting material used to lift footwear and fingerprint impressions that have been treated with fingerprint powder.

Midsole: A component positioned between the upper and the outsole on some footwear to provide cushioning and support.

Mold: A metal cavity containing a footwear sole or tire tread design used to produce footwear or tires. 
Mold characteristic: Those design and size features of a particular mold.

Mold cure: Term used by tire manufacturers to describe the vulcanization of a tire in the molding process.

Mold parting line: The dividing line between two halves of a shell mold, or between the segments of a segmented mold.

Natural crepe rubber: A crude form of coagulated natural rubber having a crinkled or knobby texture.

Natural rubber: A natural product derived from latex tapped from rubber trees.

Negative impression: An impression that has resulted from the removal of a substance from a substrate by a footwear outsole or tire tread.

Negative control: Confirmation of no color change in the absence of blood.

Noise treatment: The mixed arrangement of tread block sizes used by the tire industry to reduce noise generated by tires.

Notches: Small void areas that extend off of grooves or slots of a tire design but don't fully cross the rib or tread block.

Oblique angle: Angle between 0 and 90 degrees.

Oblique lighting: Illumination from a light source that is at a low angle of incidence, or even parallel, to the surface of the item. (Also known as side lighting.)

Offset: The distance from the wheel's centerline to the wheel's mounting surface. Offset is measured as positive or negative.

Open pour molding: A method of making outsoles utilizing polyurethane (PU). The mold is filled by pouring the PU into the mold cavity and then closing the mold. Single unit soles are made by pouring the PU into the mold and allowing the sole to harden. Direct attached soles can be made utilizing this process. See Direct attach.

Outsole/sole: The bottom portion of the footwear that comes into contact with the ground.

Outsole/sole design: A term used to describe a specific pattern or arrangement of design elements on an outsole typically associated with a manufacturer and having a name and/or style number. (Also referred to as tread design.)

Partial or fragmented impression: An impression that does not represent the entire footwear outsole or tire tread.

Patent impression: An impression visible to the naked eye. 
Pattern: See Design.

Photo log: A written record of photographs taken at the crime scene.

Physical size: The dimensions, shapes, spacing and relative positions of the footwear outsole design components and tire tread blocks (not the same as the manufacturer's footwear or tire size). Physical size is a class characteristic.

Pitch length: Circumferential length allotted for a tire tread block.

Pitch sequence: The arrangement of tire tread blocks of varied pitch lengths to reduce tire noise.

Ply: Rubber-coated parallel cord fabric placed over the liner forming the tire carcass.

Pneumatic tire: A tire filled with air under pressure.

Polarized lighting: Illumination consisting of light rays with a single propagation direction and a single vibration direction. Polarized light is produced by the use of a polarizing filter.

Polyurethane (PU): A polyester or polyether-based polymer used in both the outsoles and midsoles of footwear.

Polyvinylsiloxane: Dental casting material formulated to render fine detail.

Polyvinyl chloride (PVC): A thermoplastic polymer used in footwear outsoles.

Position and orientation of wear: The location and direction of an area of erosion on a footwear outsole or tire tread. Examples of location of wear include wear along the medial edge of the footwear outsole and wear along the outer edge of a tire tread. The position and orientation of wear can change as a footwear outsole or tire tread is worn.

Positive impression: See Transfer impression.

Positive control: Confirmation of a color change in the presence of blood.

Pressed sole: A sole made in the compression mold.

Printer's ink: A highly toned oil-based black ink. Printer's inks that set up in two to four hours are often used in the production of full circumference known tire impressions.

Questioned impression: An impression of an unknown footwear or tire located and recovered from a crime scene.

Radial ply tire: A tire whose plies run from bead to bead at right angles to the centerline of the tread. 
Randomly acquired characteristic: A feature on a footwear outsole or tire tread resulting from random events including, but not limited to: cuts, scratches, tears, holes, stone holds, abrasions and the acquisition of debris. The position, orientation, size and shape of these characteristics contribute to the uniqueness of a footwear outsole or tire tread. Randomly acquired characteristics are essential for an identification of a particular item of footwear or tire as the source of an impression.

Release agent: Any product that prevents soil from adhering to the cast.

Residue impression: Formed by the deposition of a substance from the footwear or tire onto another surface.

Retreaded tire: A used tire to which a new tread has been added.

Release agent: Any product that prevents soil from adhering to a cast.

Rib: Row of continuous rubber or disconnected tire tread blocks that run circumferentially around a tire to form the tread pattern, further distinguished as center, intermediate, or shoulder ribs.

Rim diameter: The diameter of the rim that supports the tire bead and is expressed in inches, such as 13", 16", 16.5 " etc.

Ritz Stick@: Device for measuring foot length and width.

Roller transport film: A seven-mil Estar film base material designed to wet rollers and pick up loose particles on all types of roller transport photo-processing machines used along with fingerprint powder to produce known impressions of footwear and tires.

Rolling circumference: The linear distance traveled by a tire in one revolution under load.

Schallamach pattern / Feathering: Microscopic patterns that develop as ridges on rubber material as a result of repeated abrasive forces. These patterns are very similar in their size and appearance to skin friction ridges and are highly individual. They continue to change rapidly as affected by continued abrasion. Schallamach patterns are randomly acquired characteristics. The term gets its name from a researcher of the same name.

Section height: The distance from the rim to the tread surface of an unloaded tire.

Section width: The distance between the sidewalls of an inflated tire, exclusive of any lettering or designs.

Segmented tire mold: A mold consisting of several segments that open and close around the tire. The sidewall plates are mounted separately. 
Shell tire mold: Also known as a two-piece mold, it consists of a top and bottom, each containing a sidewall ring and half of the full-circle tread design.

Shoe perimeter: The outer border or edge of the footwear sole that defines its overall physical size and shape. Some perimeters may be comprised of a border such as a molded border or a foxing strip.

Shoe size: The size a manufacturer designates for an item of footwear and places on a label in the footwear and/or footwear sole, and shoe box. There is not a strict dimensional relationship between a manufacturers shoe size and the length and width of the outsole.

Shoe size grading: The gradual increase or decrease in physical size and content that a manufacturer uses for each half size. In general, each half size will result in an approximate measurement change of $4.2 \mathrm{~mm}$ in length of the outsole.

Shoulder: The portion of the tire where the sidewall and tread meet.

Side-by-side: A comparison method performed by placing two or more objects adjacent to one another.

Sidewall: The portion of the tire between the shoulder and the bead that contains the tire information.

Similar: An observation that an impression shares a general likeness with a known footwear or tire. (Similar should not be confused with correspond.)

Sipes: Thin slits in a footwear outsole or tire tread to create better traction. True sipes are those that are cut into a footwear outsole during manufacture. True sipes are cut in a tire tread only after market. True sipes must be flexed to open. Imitation sipes are molded and remain open.

Slot: A lateral groove on a tire tread separating tread blocks.

Snow Print Wax ${ }^{\mathrm{TM}}$ or Snow impression wax: Aerosol waxes used to coat the surface of snow impressions prior to casting.

Specific location of wear: A defined area of erosion on a footwear outsole or tire tread. Examples of a specific location of wear are a worn tire sipe or a small area of worn stippling on a footwear outsole. Specific locations of wear may allow for a greater level of discrimination or association between questioned impressions and known footwear or tires.

Specific sole design: The precise arrangement of design elements of part or all of a footwear outsole. The precise size/shape and arrangement of design elements in an outsole of one style and manufacturer's size are normally distinguishable from other sizes of the same manufacturer's style. See Design/Size relationship. 
Sprue: The piece of material that represents the passageway where the molding material was injected into the mold to form a sole and remains attached to the outsole at that point. The sprue is removed before sale.

Sprue mark: A small circular mark left on the surface of the back of the heel of the outsole after the sprue has been removed.

Standard: See Test impression.

Stippling: A pattern hand struck onto the surface of a mold using a steel die containing a selected design. The tip of the die is small and requires numerous, often overlapping, strikes. These multiple strikes result in a fine pattern on the surface of the mold, and subsequent outsoles that come from that mold. Because of the random manner in which hand stippling is applied, it is unique to that specific mold.

Stone hold: A stone held in a recessed area of a footwear or tire that may or may not be replicated in an impression.

Sulfur: A substance used for casting snow impressions.

Sulfur cement: A reinforced modified sulfur material, available in flake form that is a safer, stronger alternative to using pure sulfur in casting snow impressions.

Superimposition: A comparison method performed by placing one object over the other.

Synthetic rubber. Any artificial elastomer that simulates the qualities of natural rubber.

Tandem: Tires set immediately one behind the other.

Tears: Fractures that have occurred in footwear outsoles or tire treads that reflect irregular edges. Tears are randomly acquired characteristics.

Test impression: An impression made from a footwear or tire used as an aid for comparison purposes.

Texture: A rough surface or shallow design added to surfaces of a mold through the process of chemical etching or stippling that is transferred to the footwear during the molding process. Texture is unique to specific molds.

Three-dimensional impression: An impression made on surfaces such as soil, sand, snow or mud with dimensions of length, width, and depth.

Tire footprint: The contact area of a tire tread against a flat surface when under load, also known as a contact patch. 
Tire profile: See Aspect ratio.

Toe bumper guard: A thick strip of rubber that, in some footwear designs, is placed around the front perimeter of the footwear surrounding the toe area.

Track width: The distance between the center points of the tires from one side of the vehicle to the other (i.e., from the center point of the right front tire to the center point of the left front tire). On a dual axle vehicle, this is the distance from the center points between the dual tires from one side of the vehicle to the other.

Transfer impression: An impression made on a two dimensional surface by a footwear or tire as a result of coming in contact with and acquiring dust, residue, blood, mud, or other materials that the footwear or tire subsequently deposits or transfers to a substrate in the form of an impression.

Tread: The designed part of the tire that comes into contact with the road.

Tread block: A shape arranged circumferentially around a tire tread that together form the tread design. See Element/Design Element.

Tread depth: A vertical measurement between the top of the tread to the bottom of the tire's deepest groove, measured in 32nds of an inch.

Tread depth gauge: A device used to measure the depth of the tire tread.

Tread design: A term used to describe a specific pattern or arrangement of design elements on a tire tread typically associated with a manufacturer and having a name and/or style number. (Also used to describe footwear outsoles.)

TreadPrint $^{\mathrm{TM}}$ : An inkless method for making tire test impressions.

Tread wear indicator: Bands of raised rubber, sometimes called "wear bars", that are $2 / 32$ of an inch above the bottom of the main grooves of a tire.

Tread width: The width of the tire tread from one edge to the other in an impression. Not to be confused with section width.

Turning diameter: The diameter of the smallest circle that is measured from the outer edge of the outermost front tire in a turn.

Two-dimensional impression: An impression with dimensions of length and width.

Unit sole: An individual heel or sole that must be glued and/or stitched to the upper.

Upper: The top portion of the footwear excluding the outsole or midsole.

Variations: Minor variables that normally exist between repetitive impressions of the same footwear or tire. 
Vent: Drilled hole or gap between tire mold components allowing for the release of air during mold cure.

Vulcanization: A process in which a rubber compound is heated under pressure causing a chemical change which transforms the rubber from a soft, tacky substance to tough, hard rubber.

Wear: Erosion of the surfaces of a footwear outsole or tire tread during use.

Wellman outsole cutting machine: A machine used to cut outsoles from unvulcanized calendered outsole material.

Wet media film: A clear drafting film, preferably with a minimum thickness of 4 mil, capable of accepting ink, which is used to obtain inked impressions of tires.

Wet origin impression: An impression formed under wet conditions including impressions consisting of residues of blood, grease, mud and other wet substances.

Wheel base: The distance between the front and rear axles of a vehicle. An approximation of this dimension can be obtained by measuring the distance from the leading edge of the rear tire track to the leading edge of the front tire track on the same side of the vehicle. 


\section{DRSA Terminology}

Table 1: Glossary of dominance-based rough set approach and rule induction terminology in relation to forensic footwear examination.

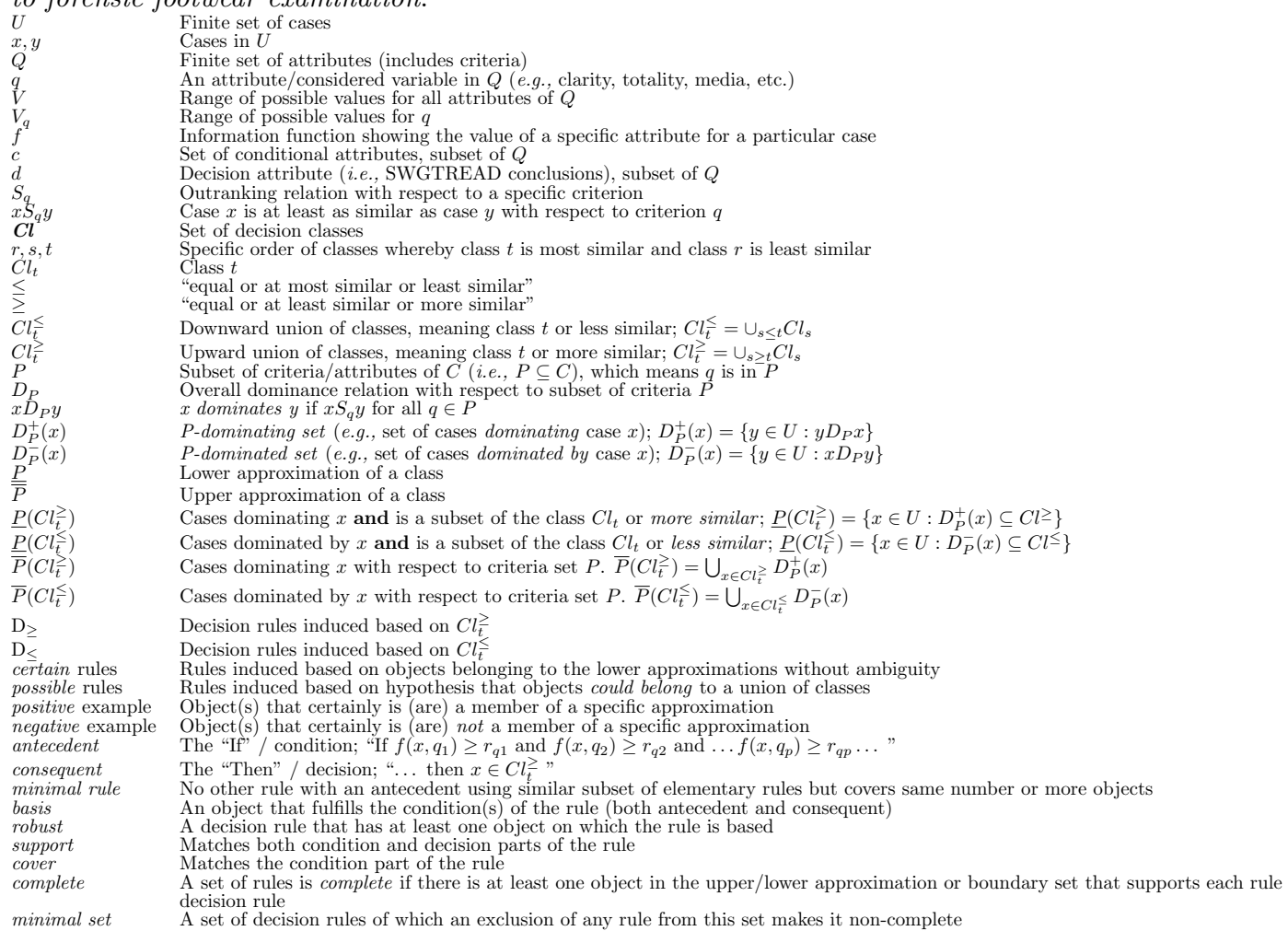




\section{Case Images}

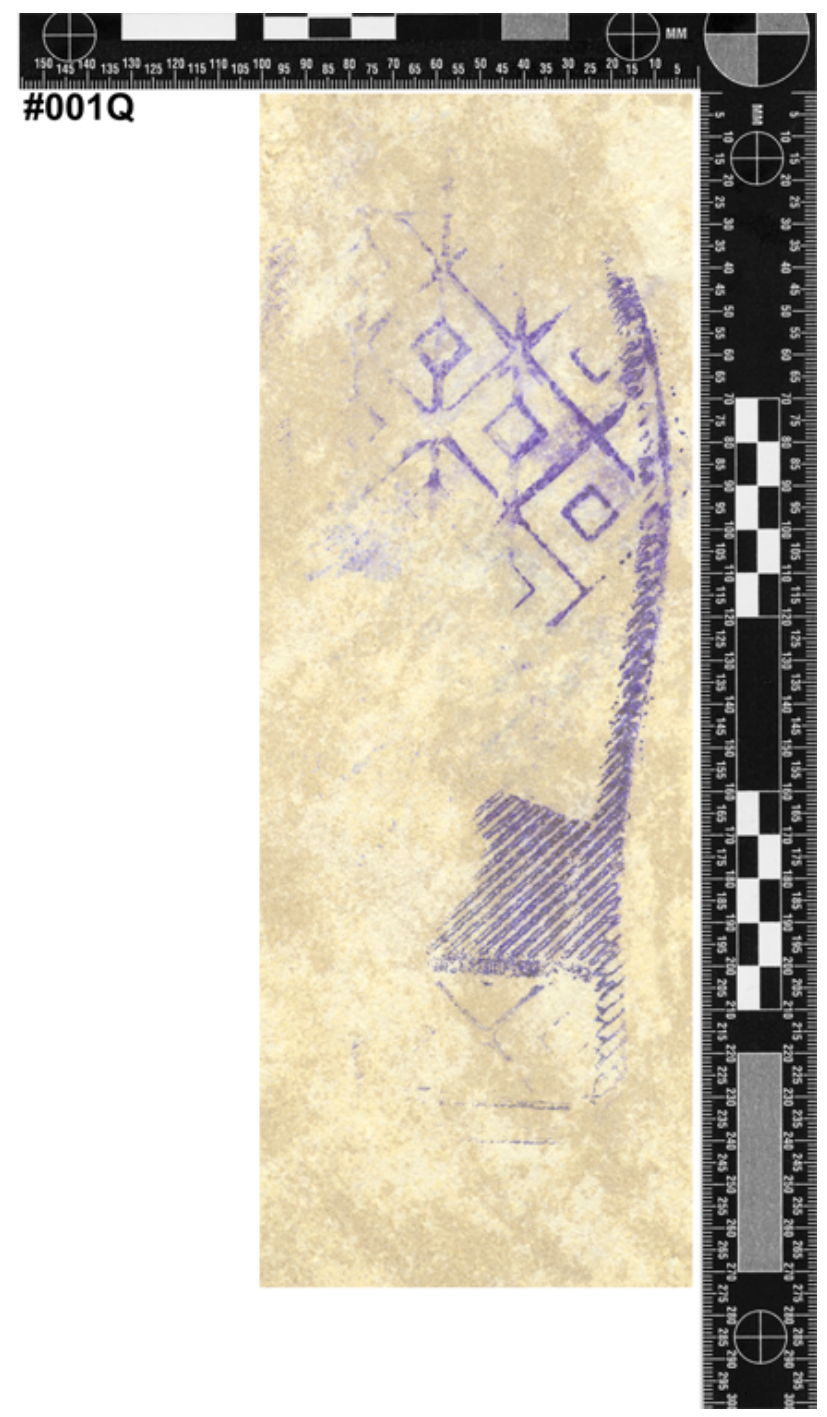

Figure D.1: Case 001: Questioned impression, 001Q. 


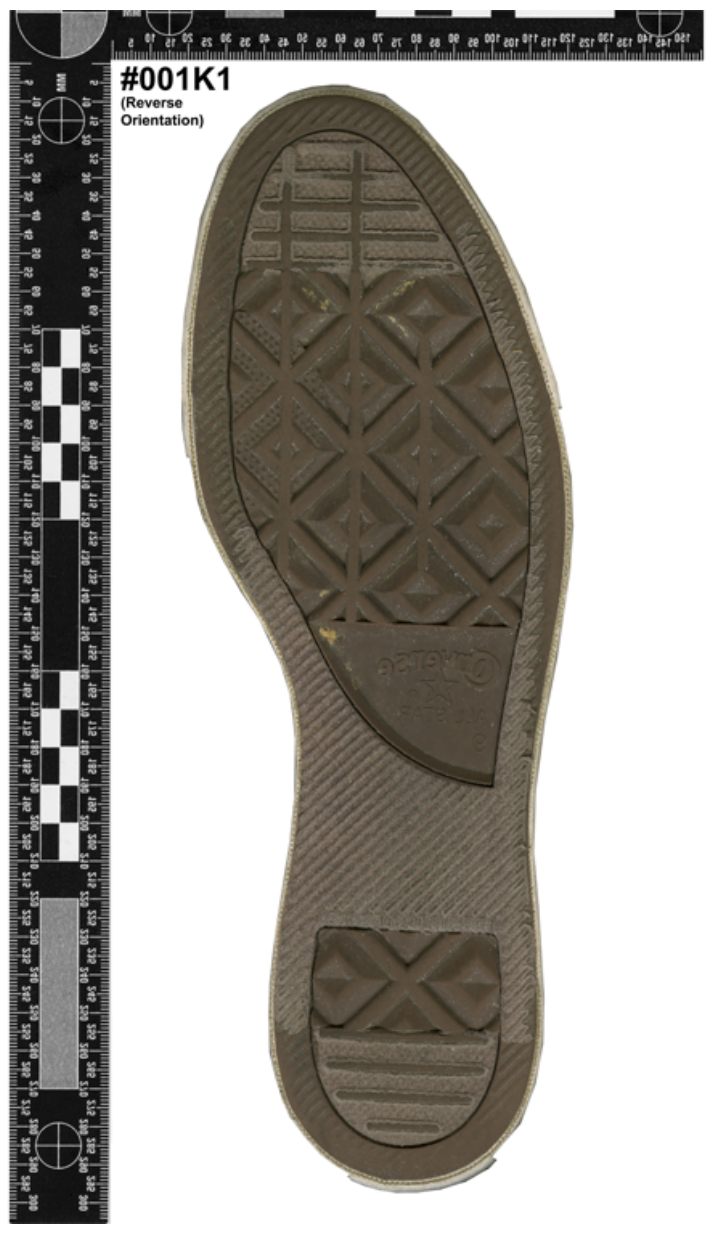

Figure D.2: Case 001: Outsole image of known footwear $1,001 K 1$.

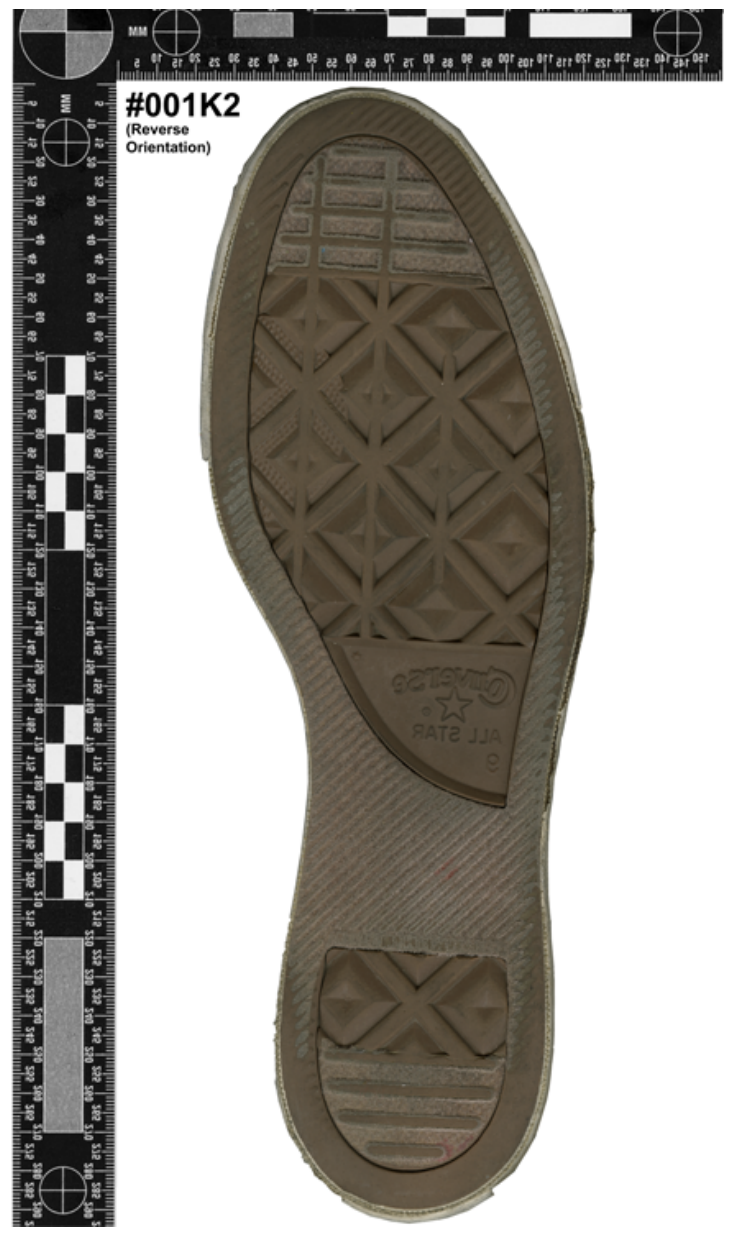

Figure D.3: Case 001: Outsole image of known footwear 2, 001K2. 


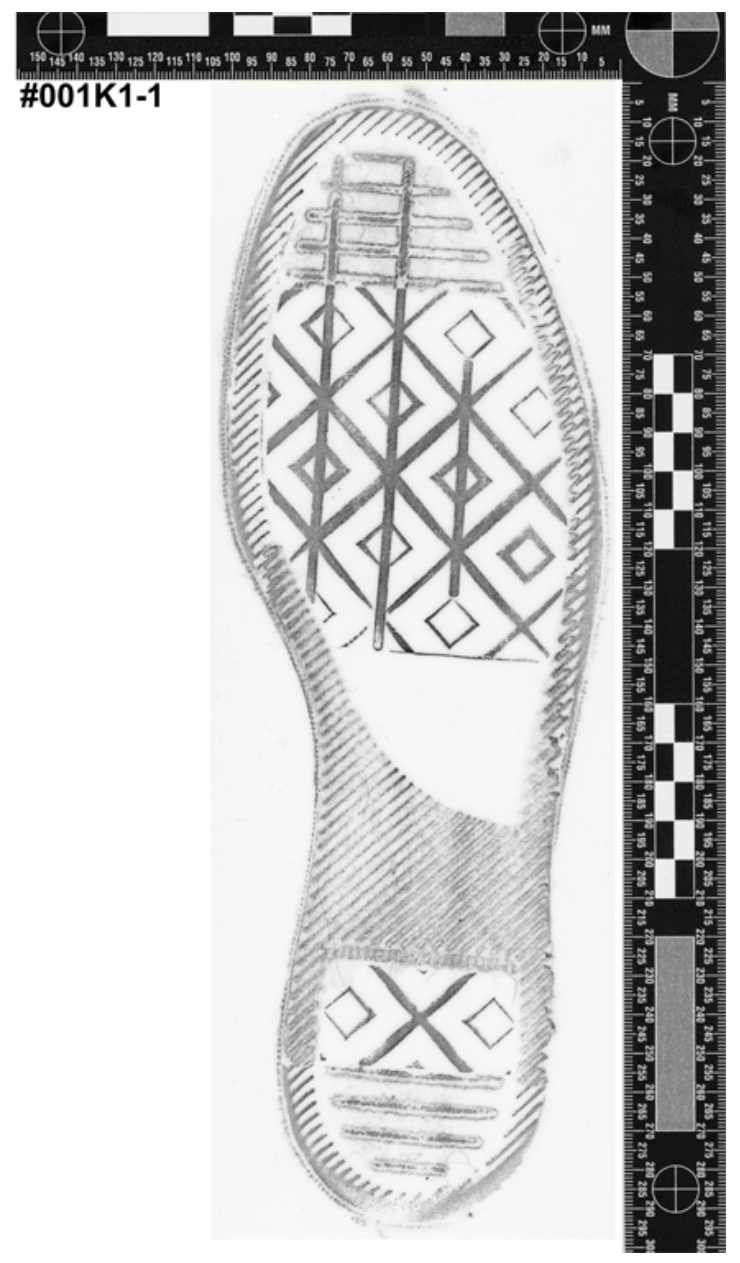

Figure D.4: Case 001: Sample Handiprint exemplar of known footwear 1, 001K1-1.

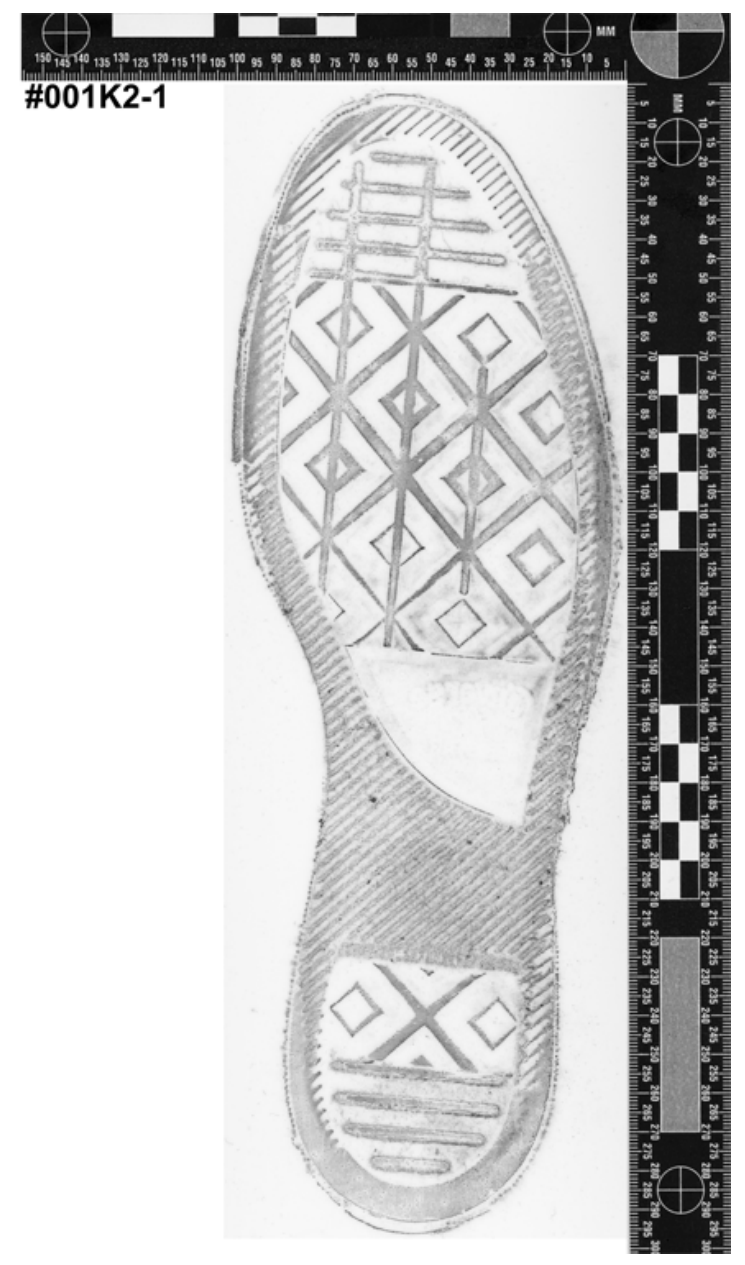

Figure D.5: Case 001: Sample Handiprint exemplar of known footwear 2,001K2-1. 


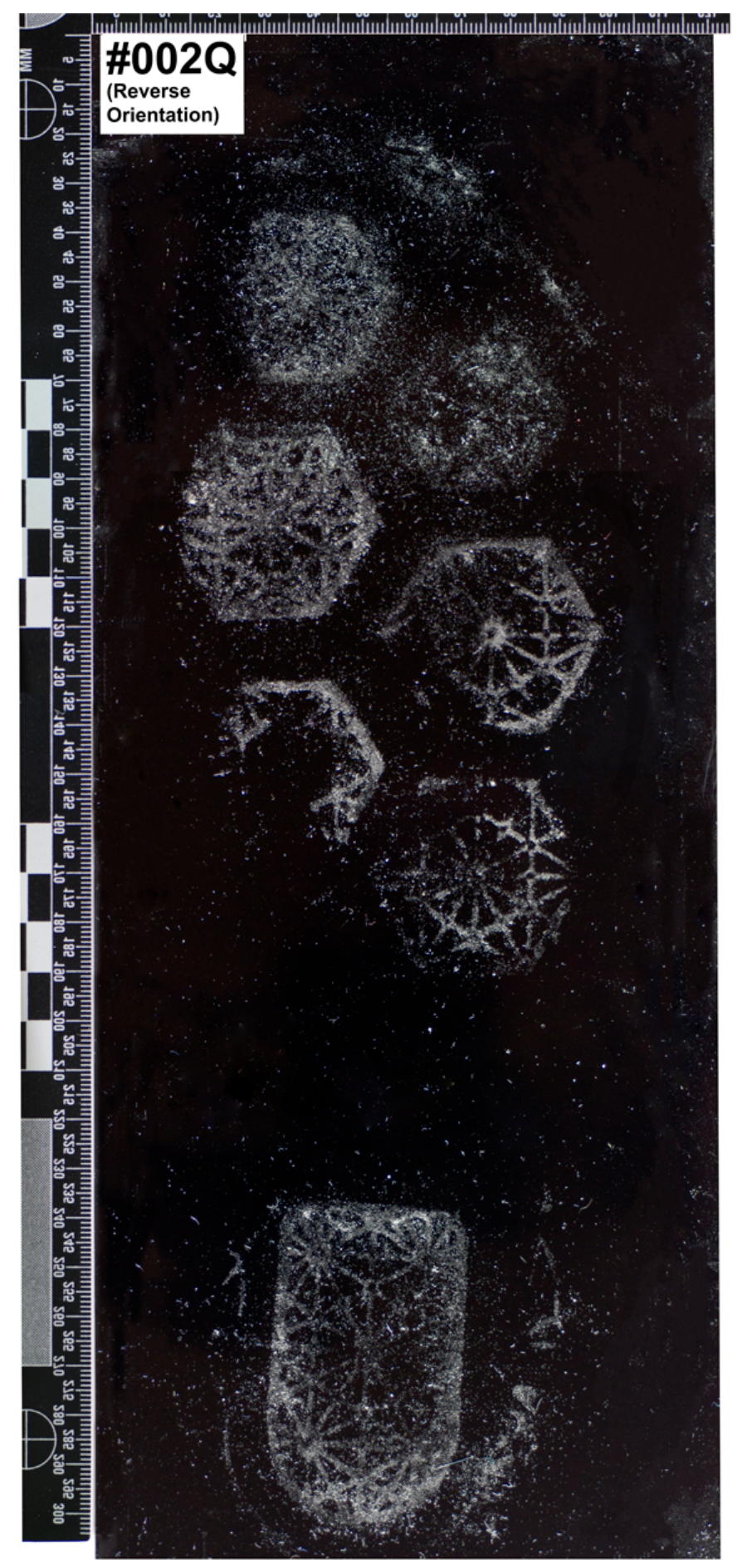

Figure D.6: Case 002: Questioned impression, 002Q. 


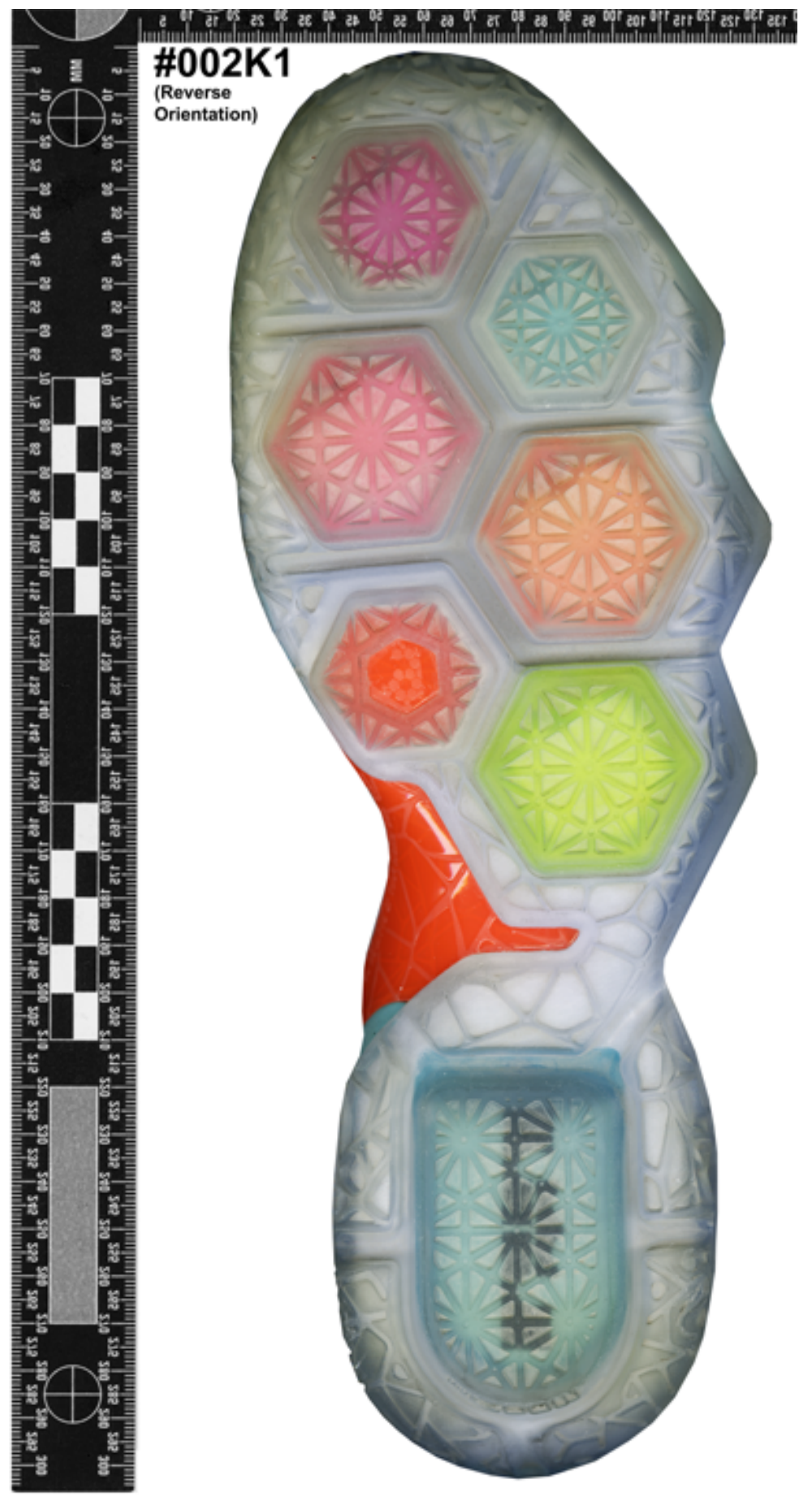

Figure D.7: Case 002: Outsole image of known footwear 1, 002K1. 


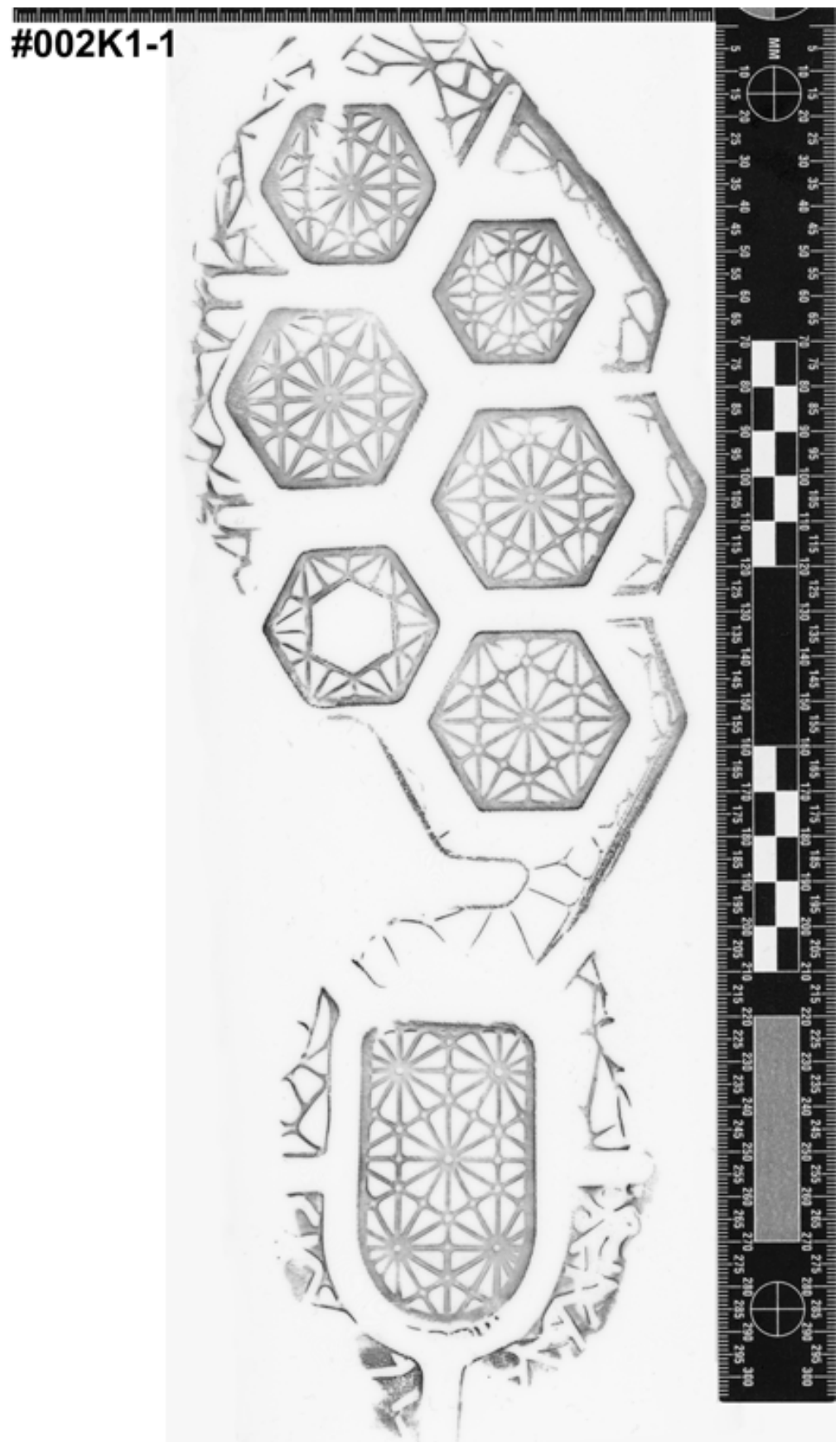

Figure D.8: Case 002: Sample Handiprint exemplar of known footwear 1, 002K1-1. 


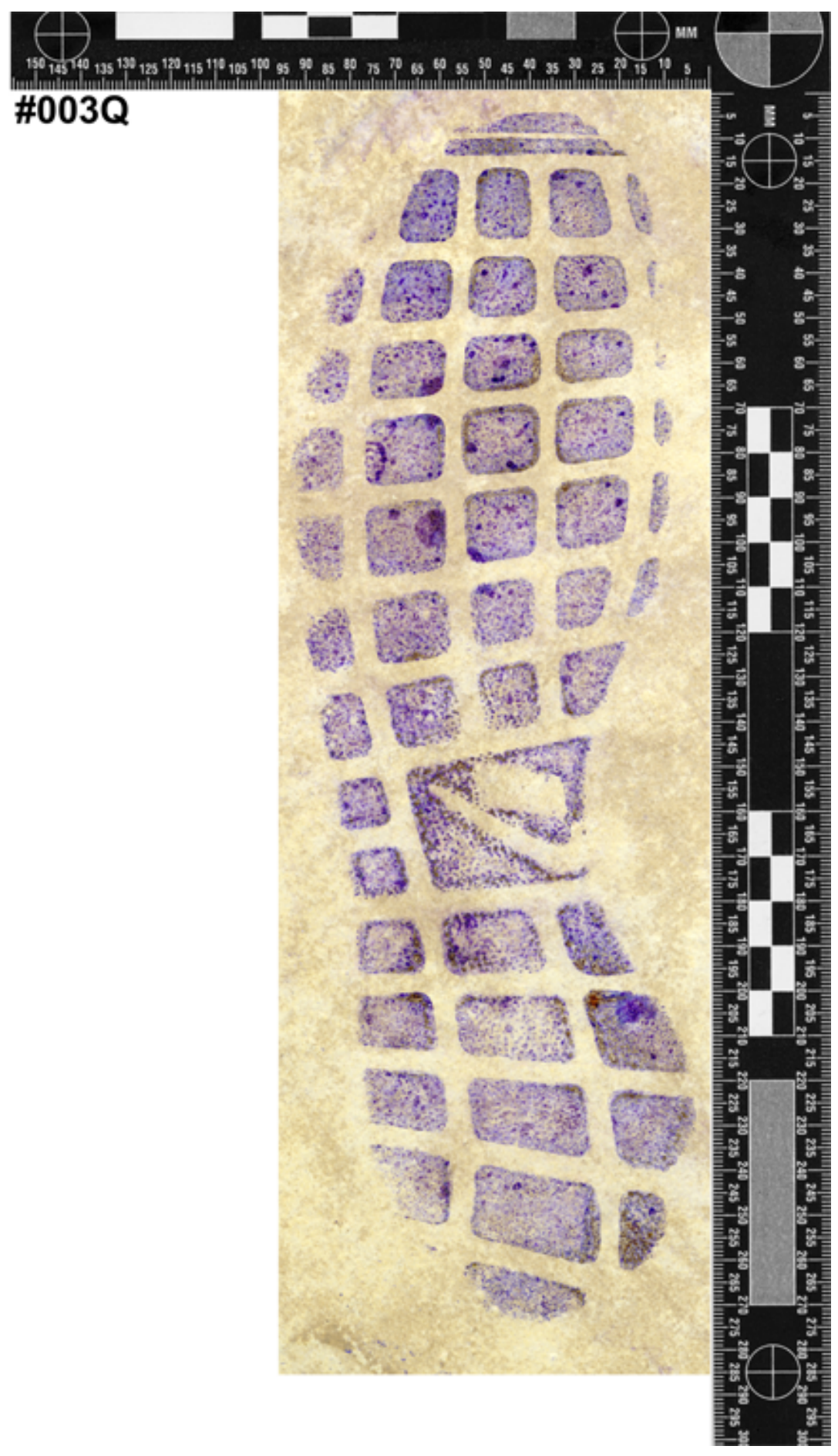

Figure D.9: Case 003: Questioned impression, 003Q. 


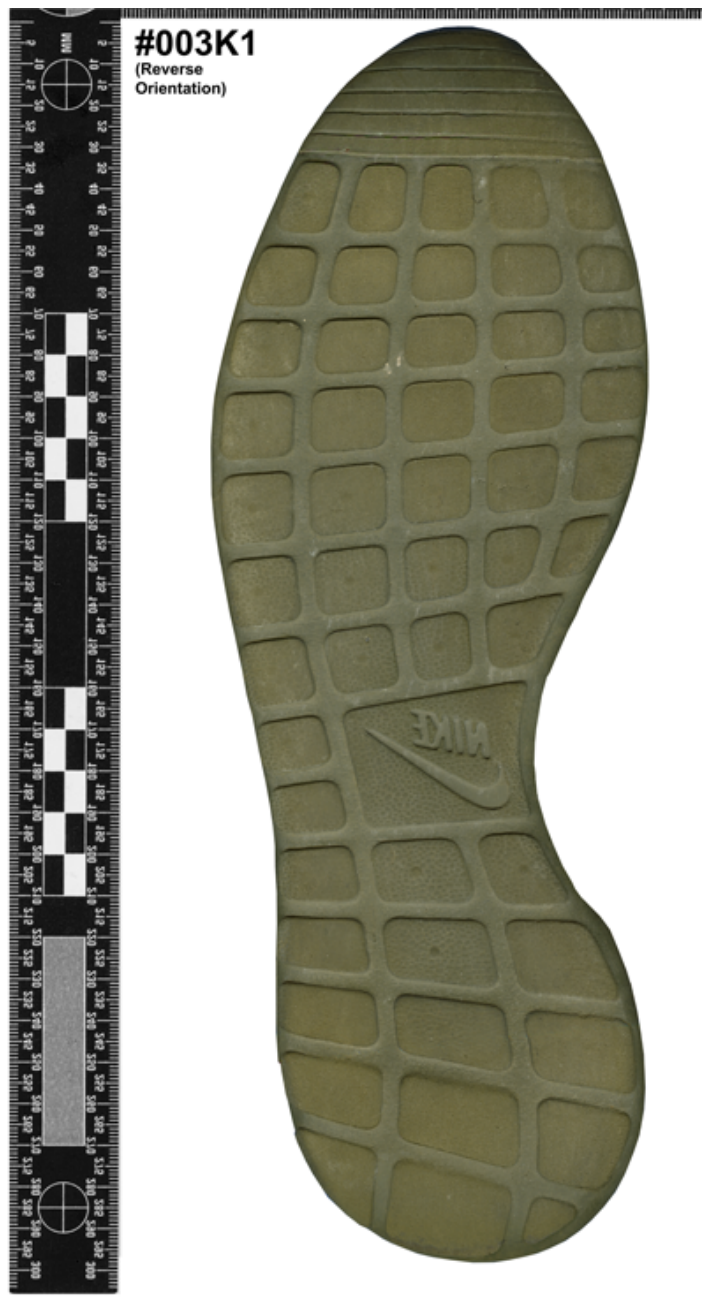

Figure D.10: Case 003: Outsole image of known footwear 1, 003K1.

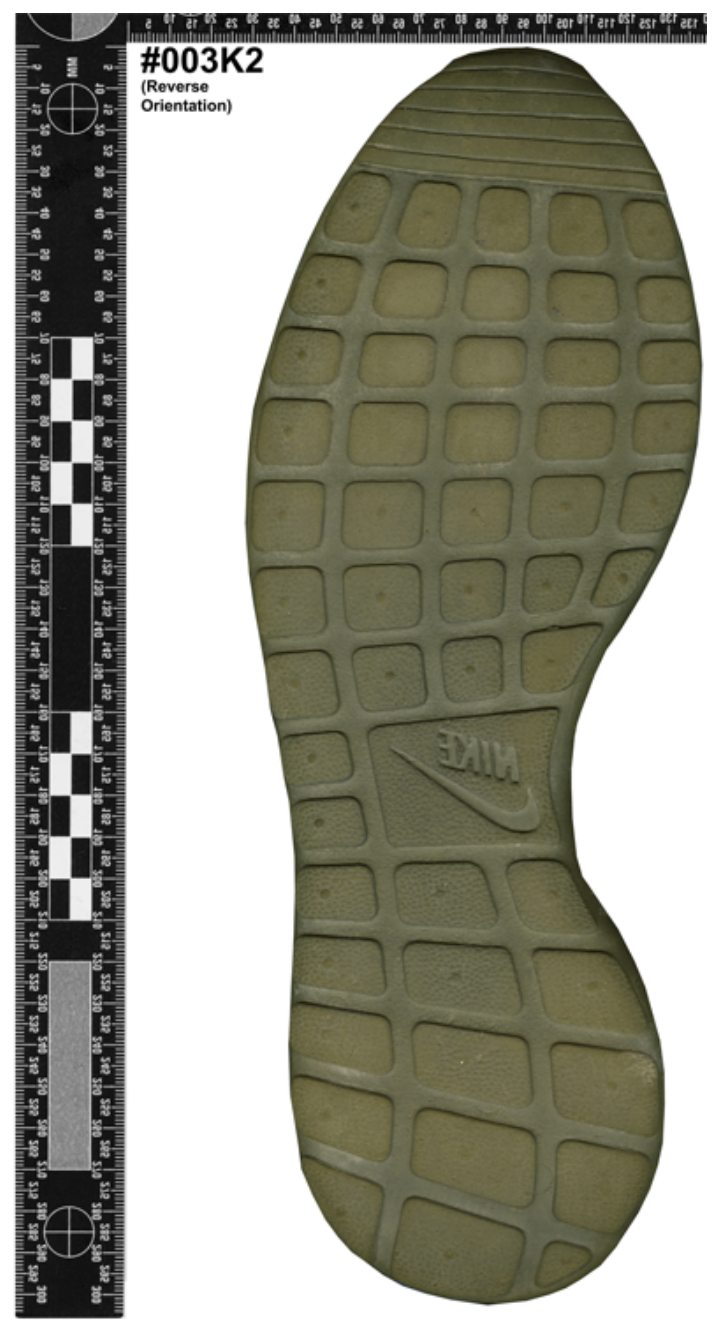

Figure D.11: Case 003: Outsole image of known footwear 2, 003K2. 


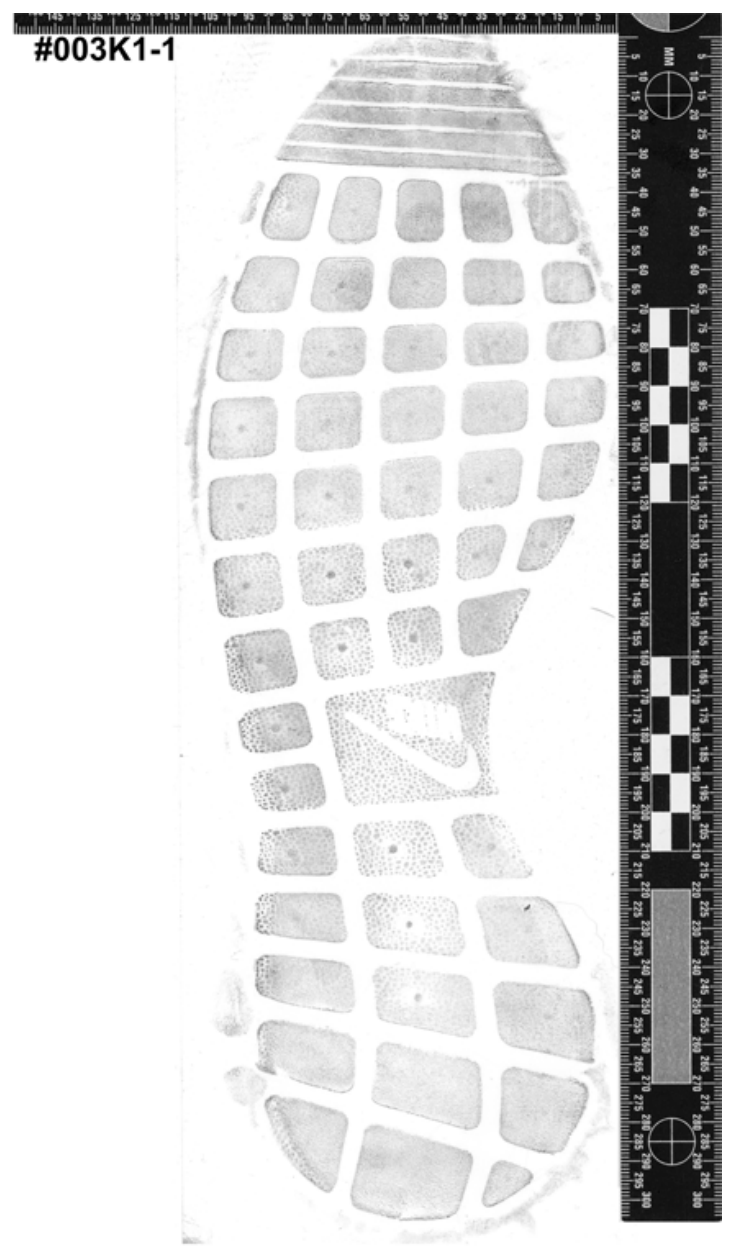

Figure D.12: Case 003: Sample Handiprint exemplar of known footwear 1, 003K1-1.

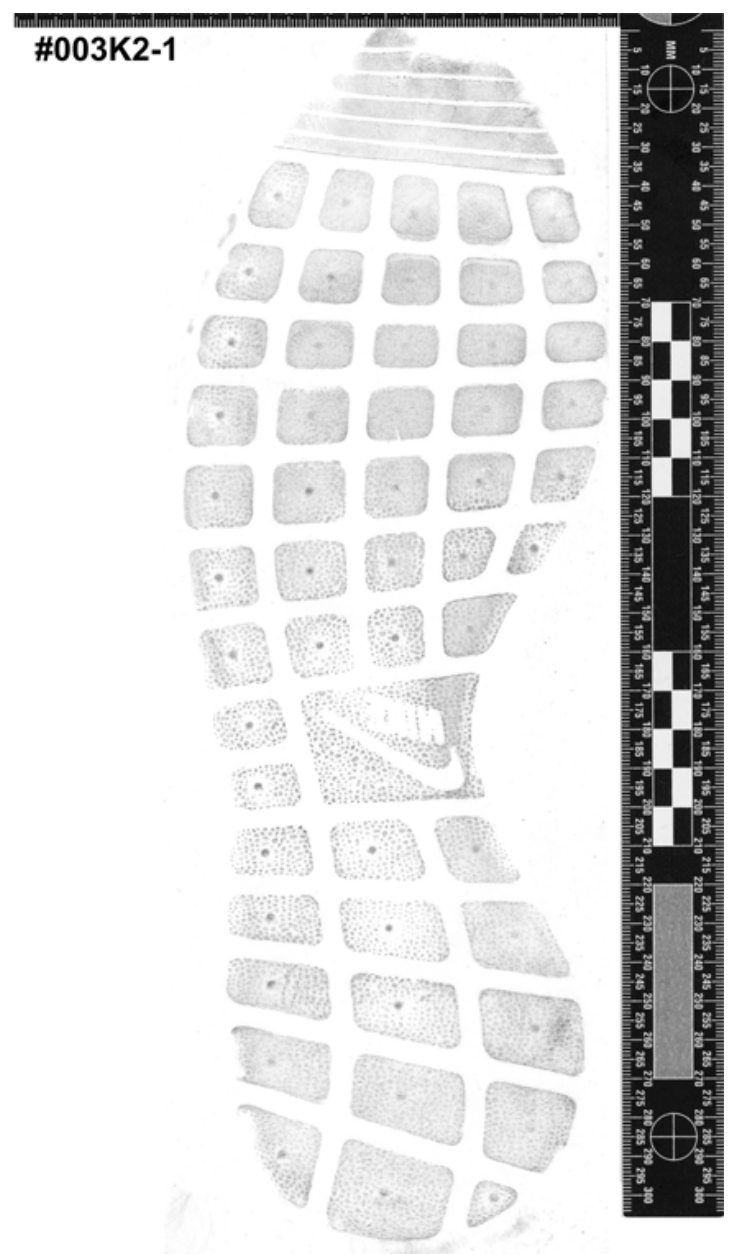

Figure D.13: Case 003: Sample Handiprint exemplar of known footwear 2, 003K2-1. 


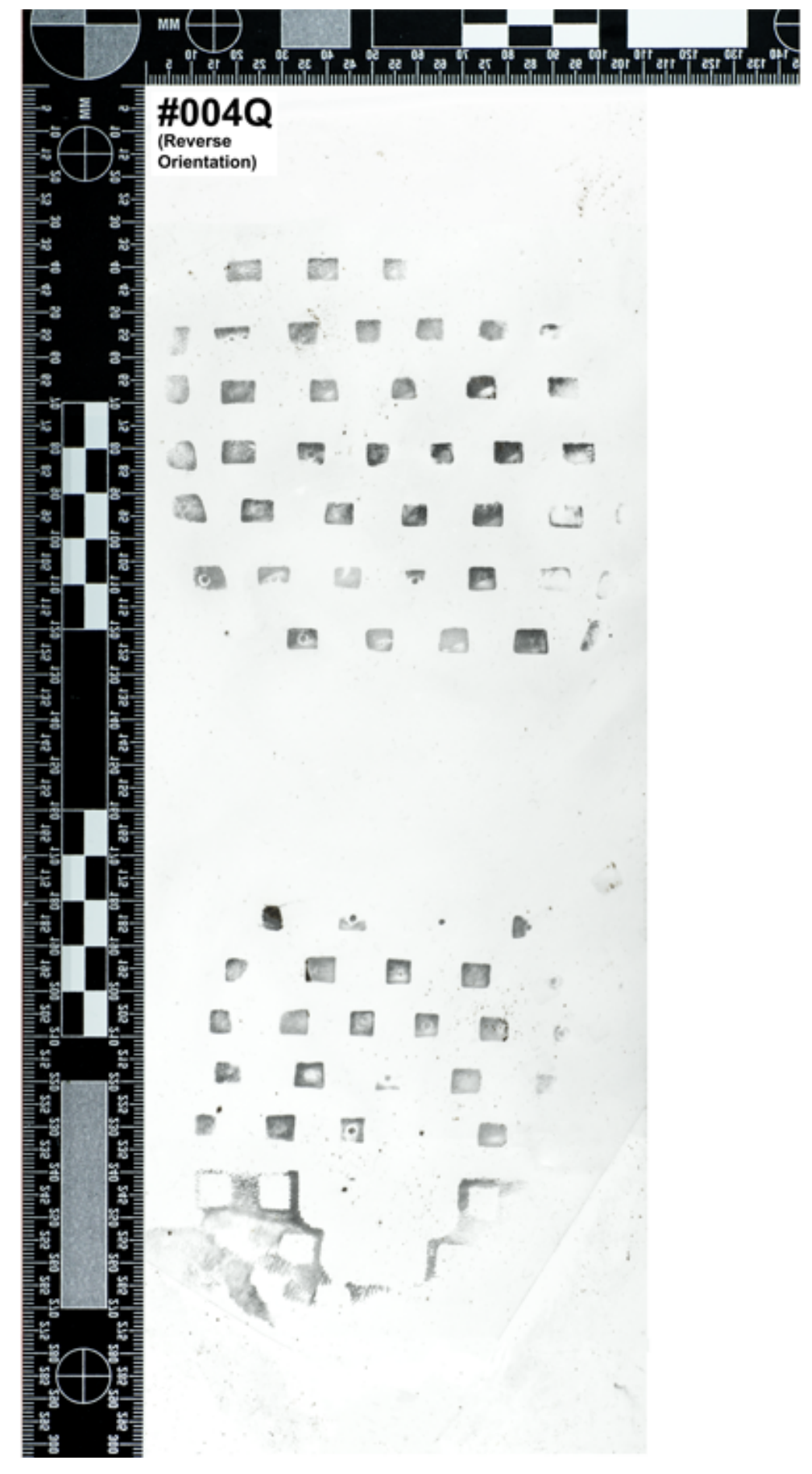

Figure D.14: Case 004: Questioned impression, 004Q. 


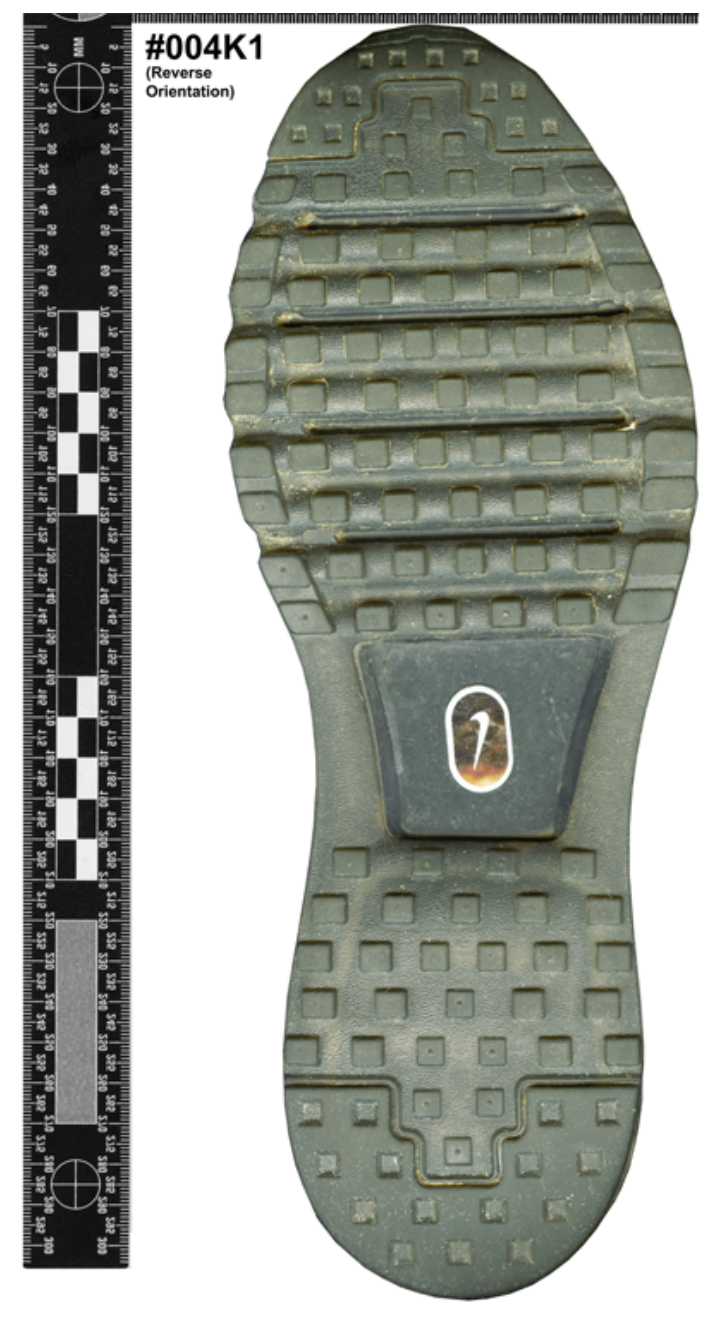

Figure D.15: Case 004: Outsole image of known footwear $1,004 \mathrm{~K} 1$.

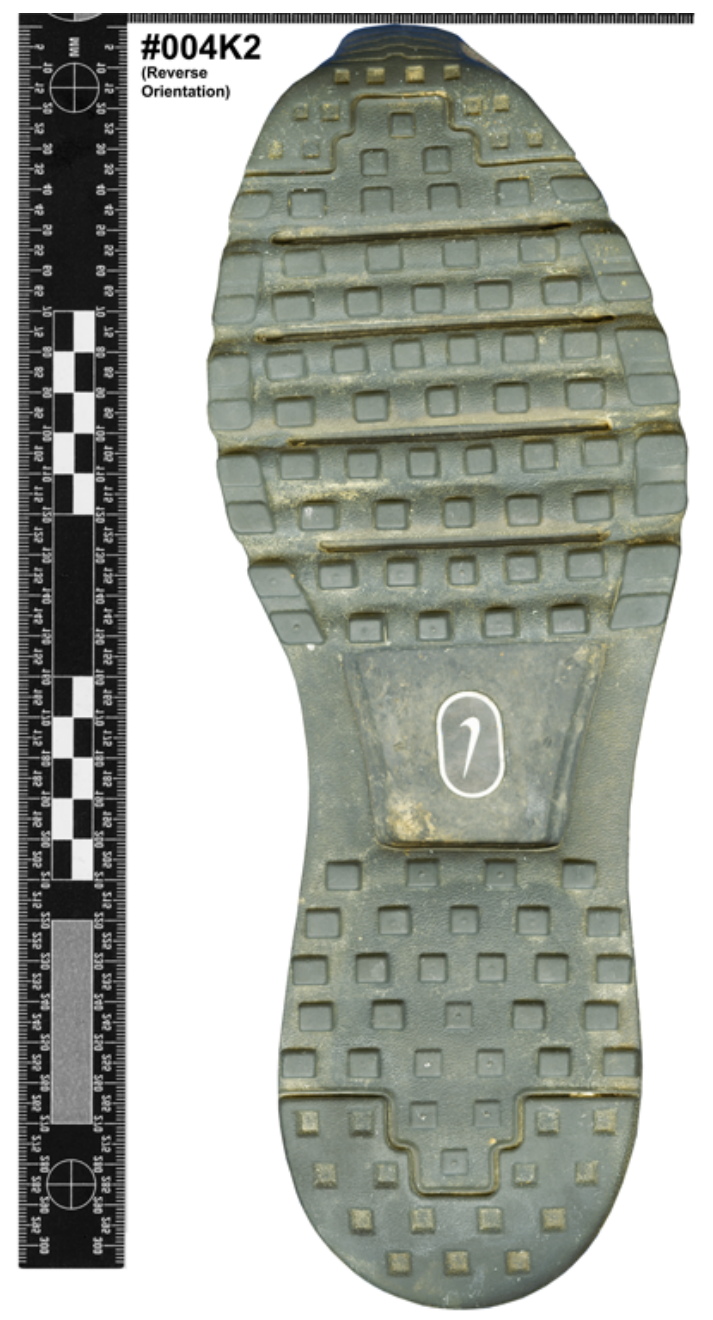

Figure D.16: Case 004: Outsole image of known footwear 2, 004K2. 


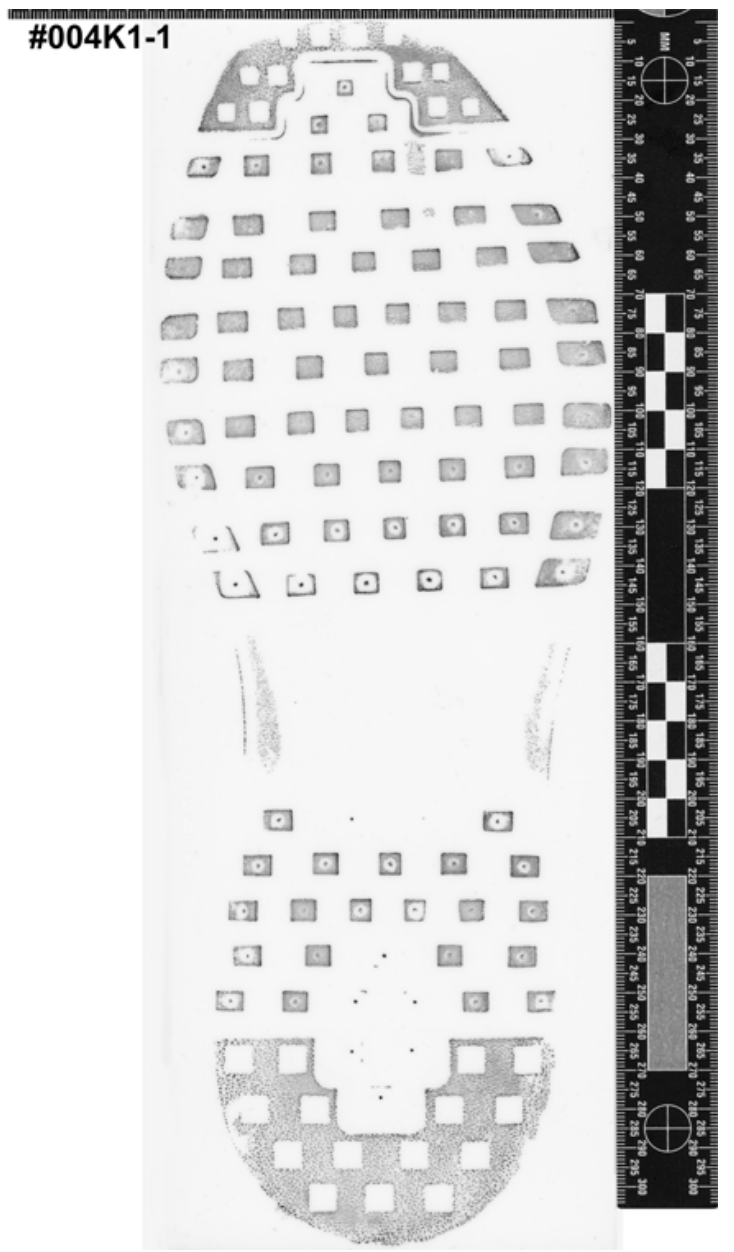

Figure D.17: Case 004: Sample Handiprint exemplar of known footwear 1, 004K1-1.

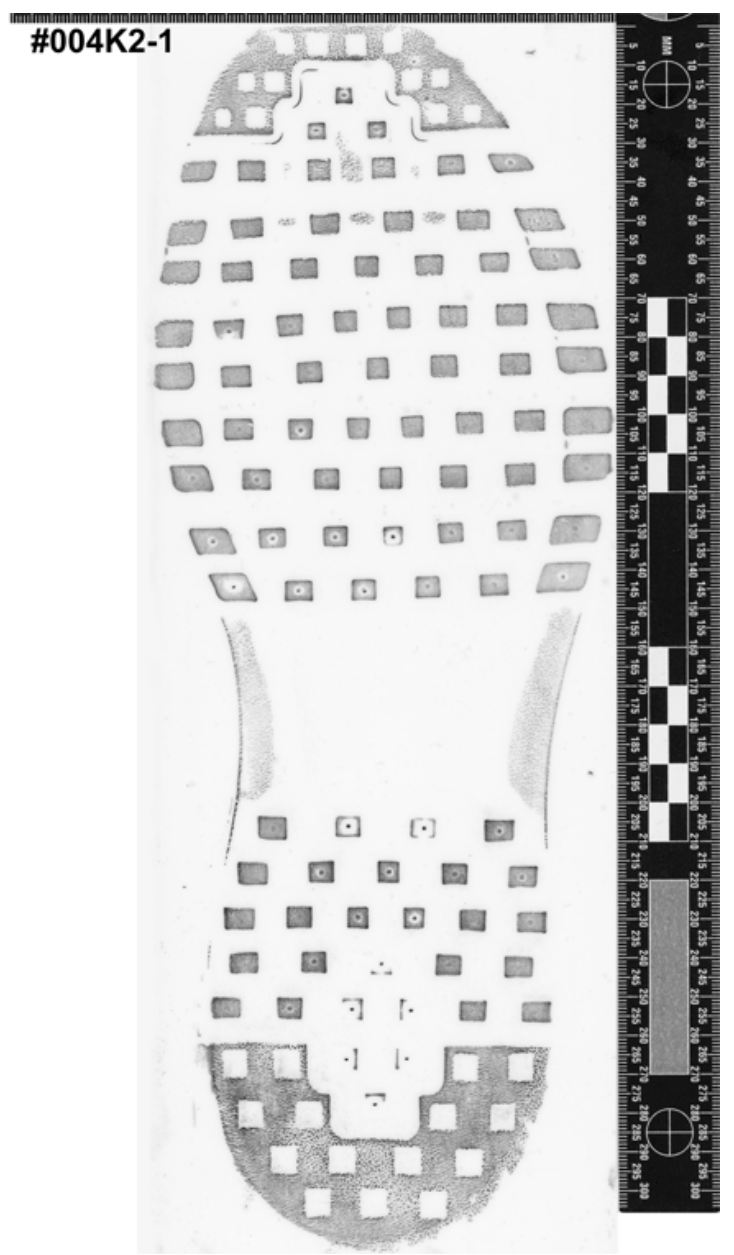

Figure D.18: Case 004: Sample Handiprint exemplar of known footwear 2, 004K2-1. 


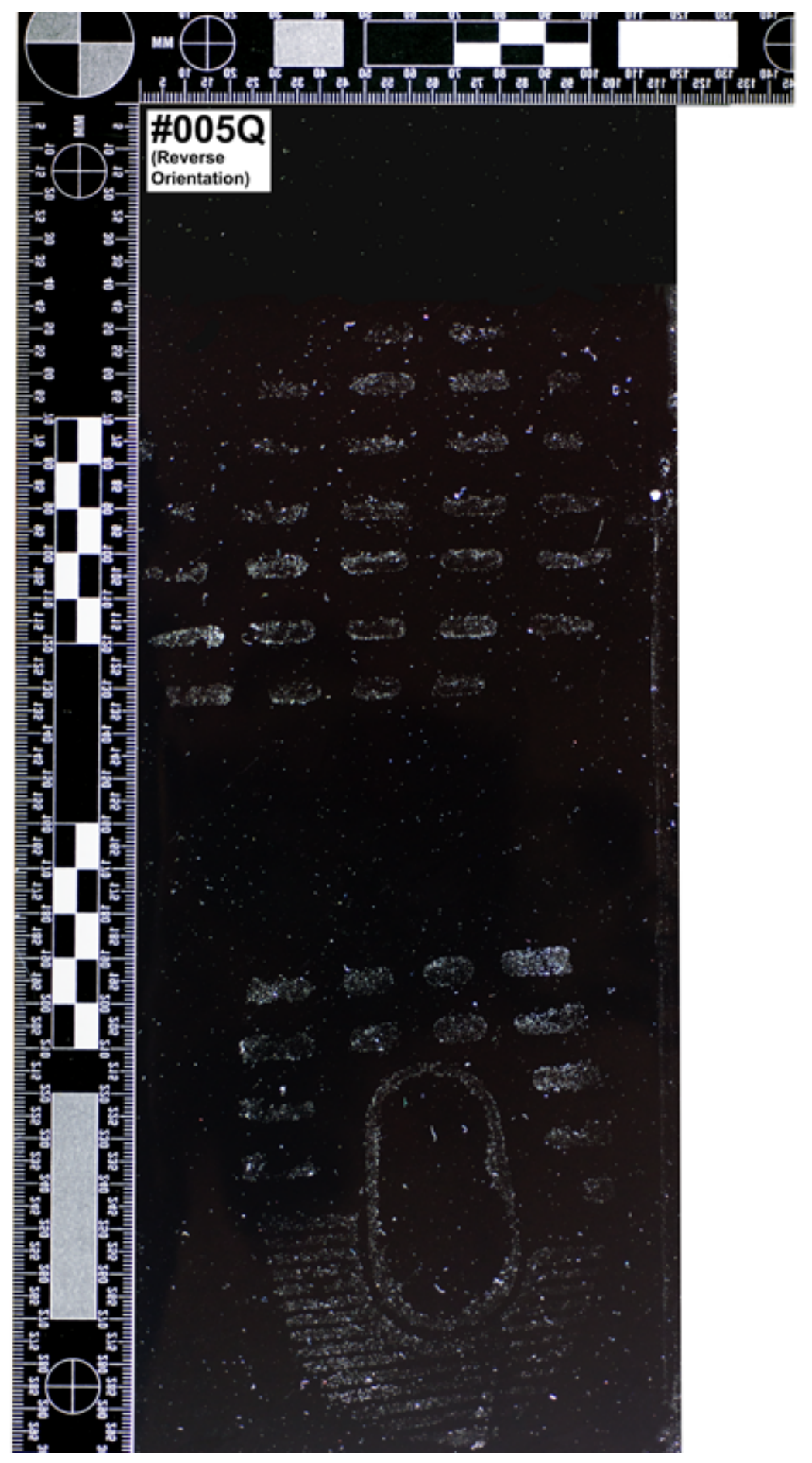

Figure D.19: Case 005: Questioned impression, 005Q. 


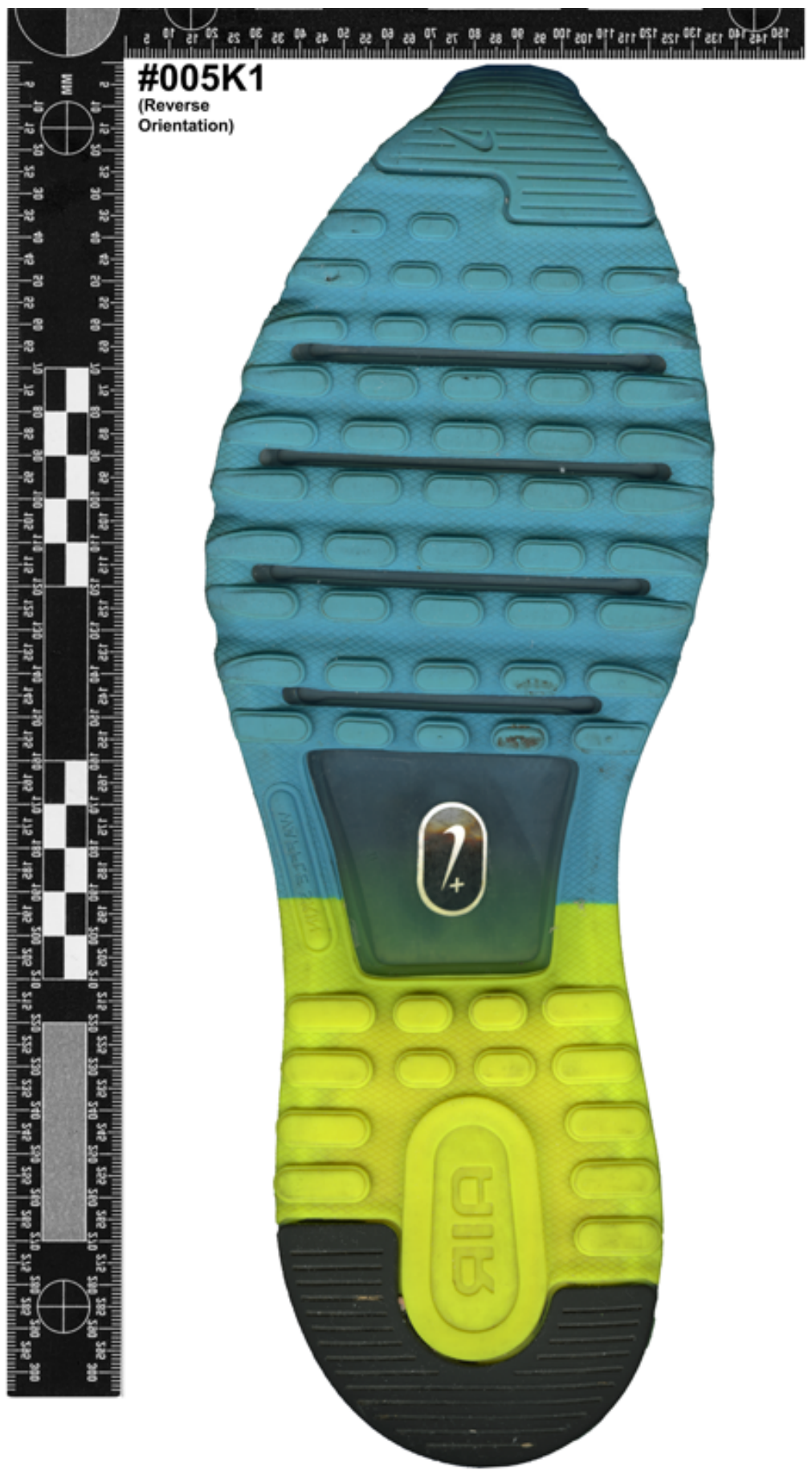

Figure D.20: Case 005: Outsole image of known footwear 1, 005K1. 


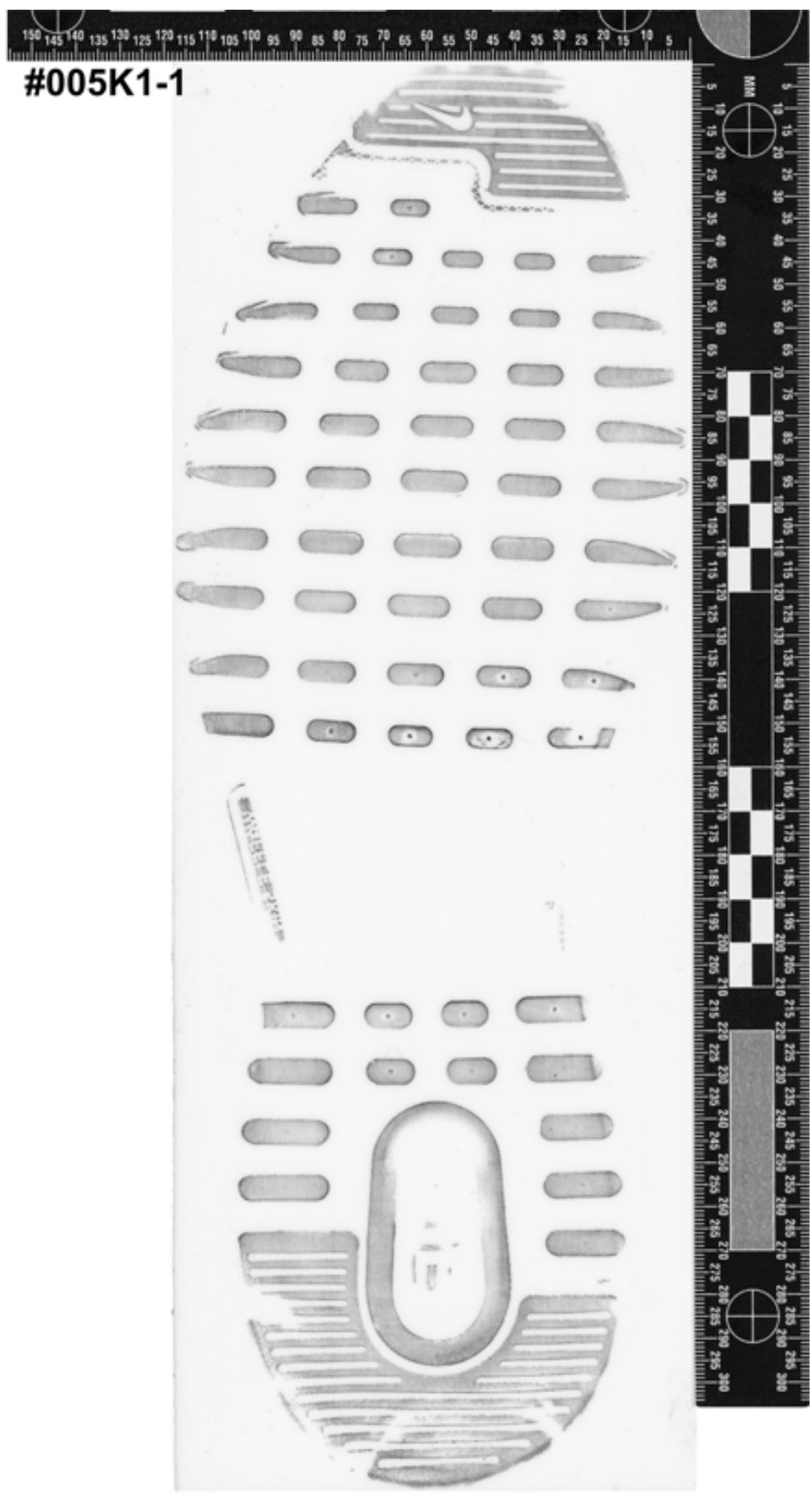

Figure D.21: Case 005: Sample Handiprint exemplar of known footwear 1, 005K1-1. 


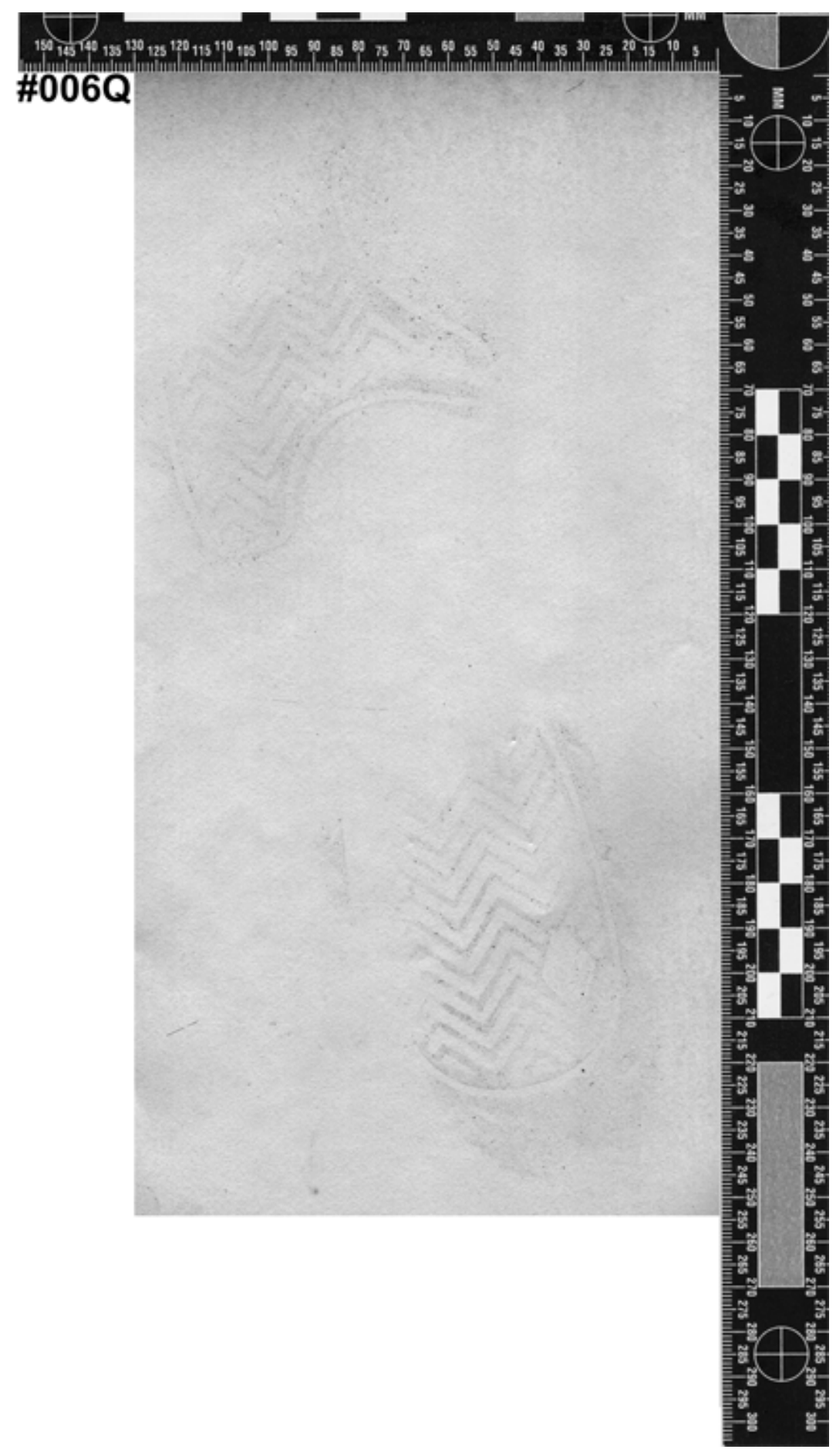

Figure D.22: Case 006: Questioned impression, 006Q. 


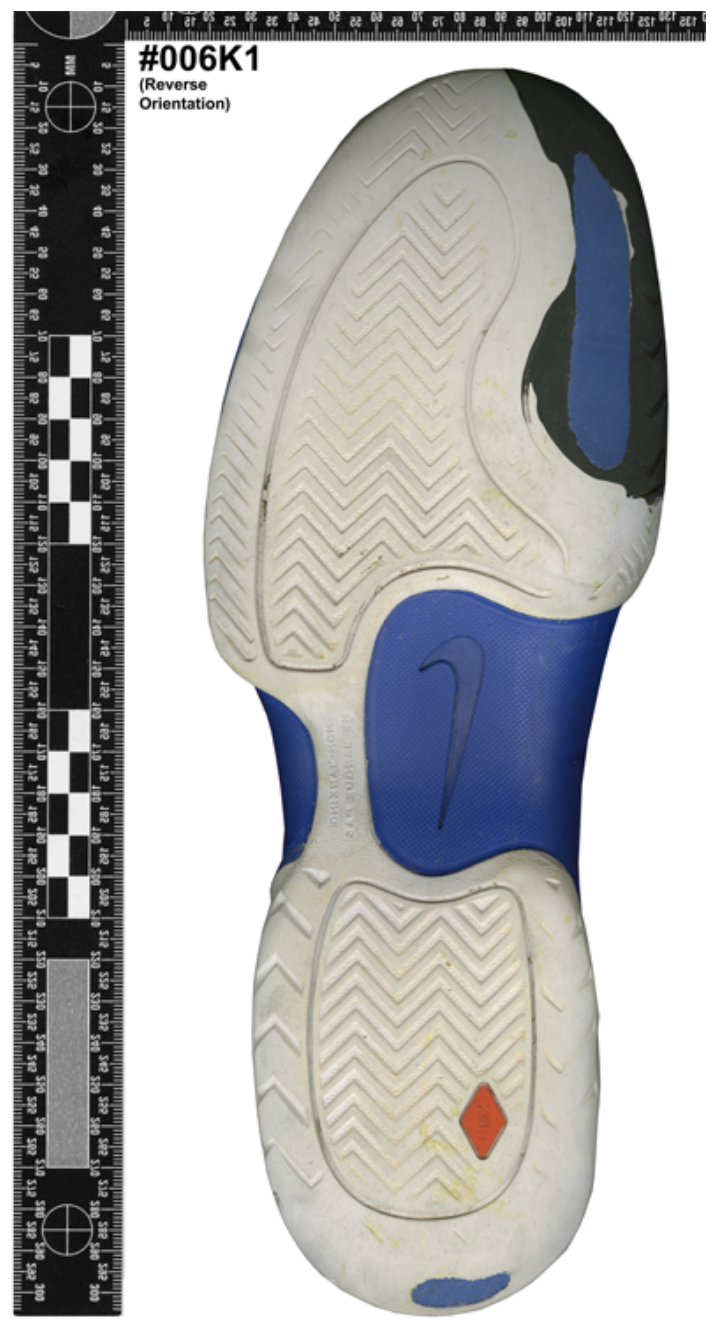

Figure D.23: Case 006: Outsole image of known footwear $1,006 \mathrm{~K} 1$.

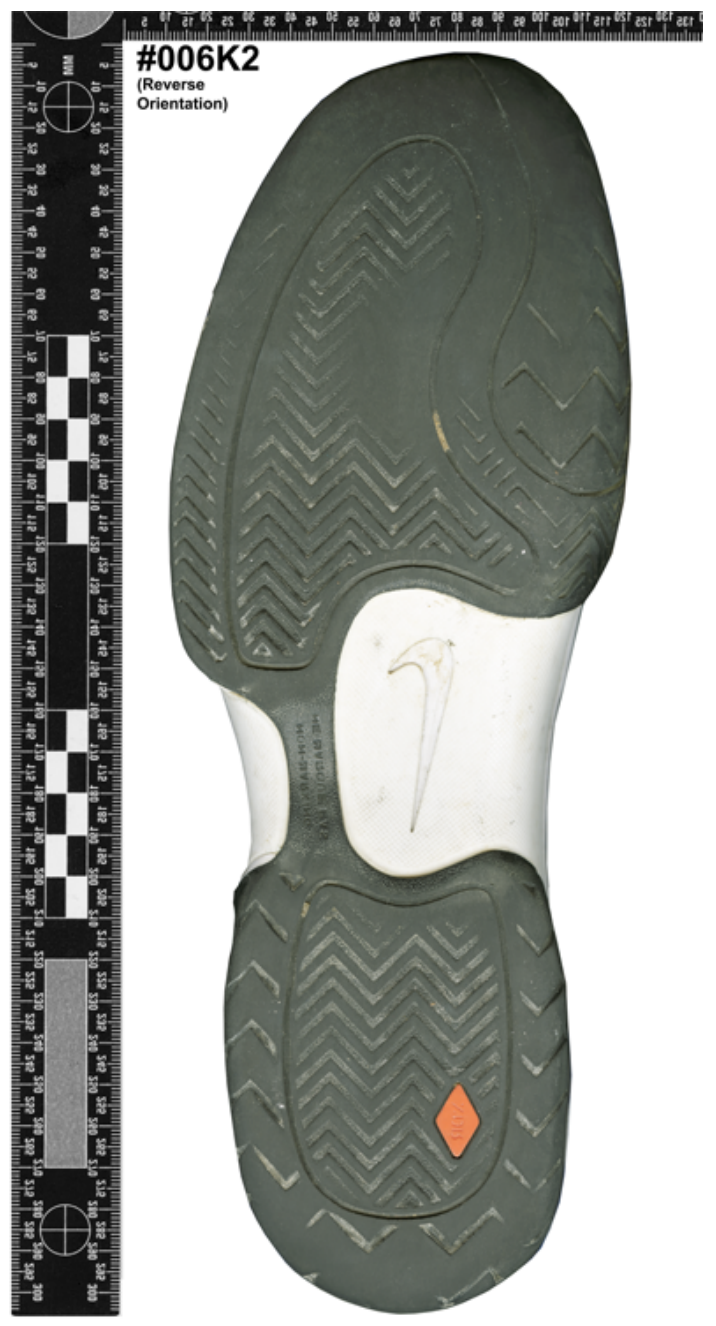

Figure D.24: Case 006: Outsole image of known footwear 2, 006K2. 


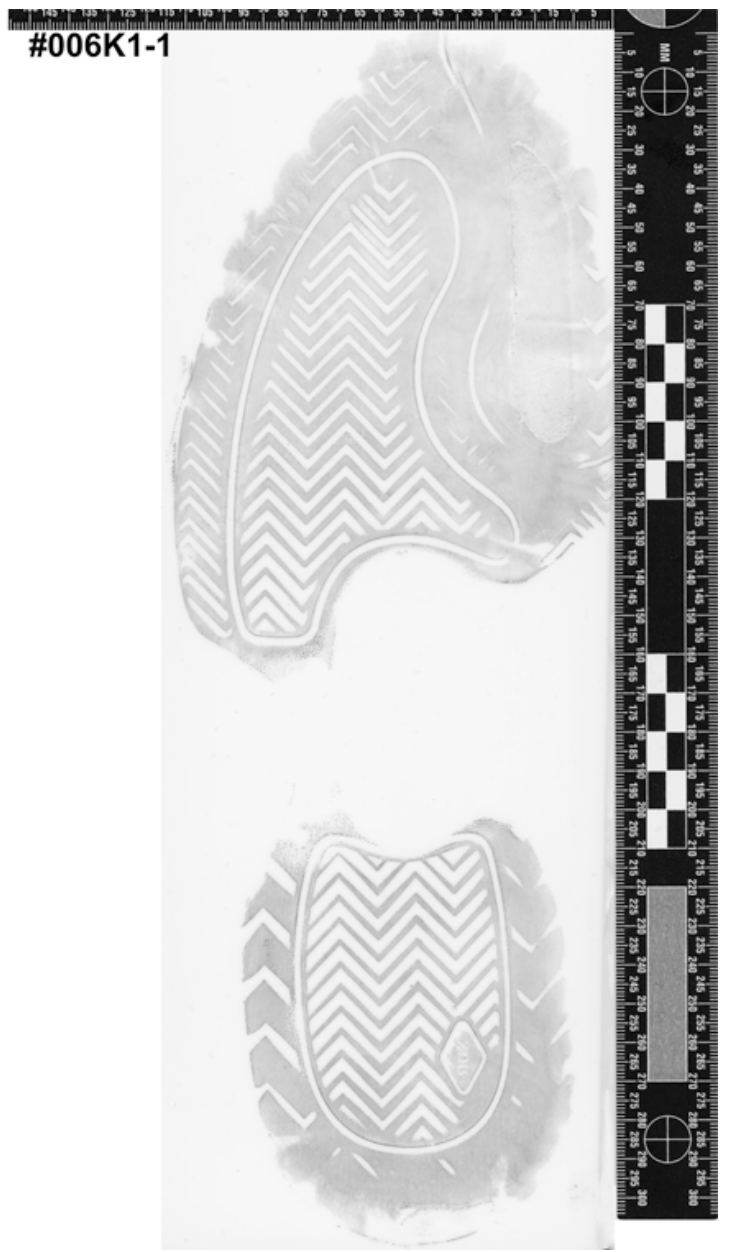

Figure D.25: Case 006: Sample Handiprint exemplar of known footwear 1, 006K1-1.

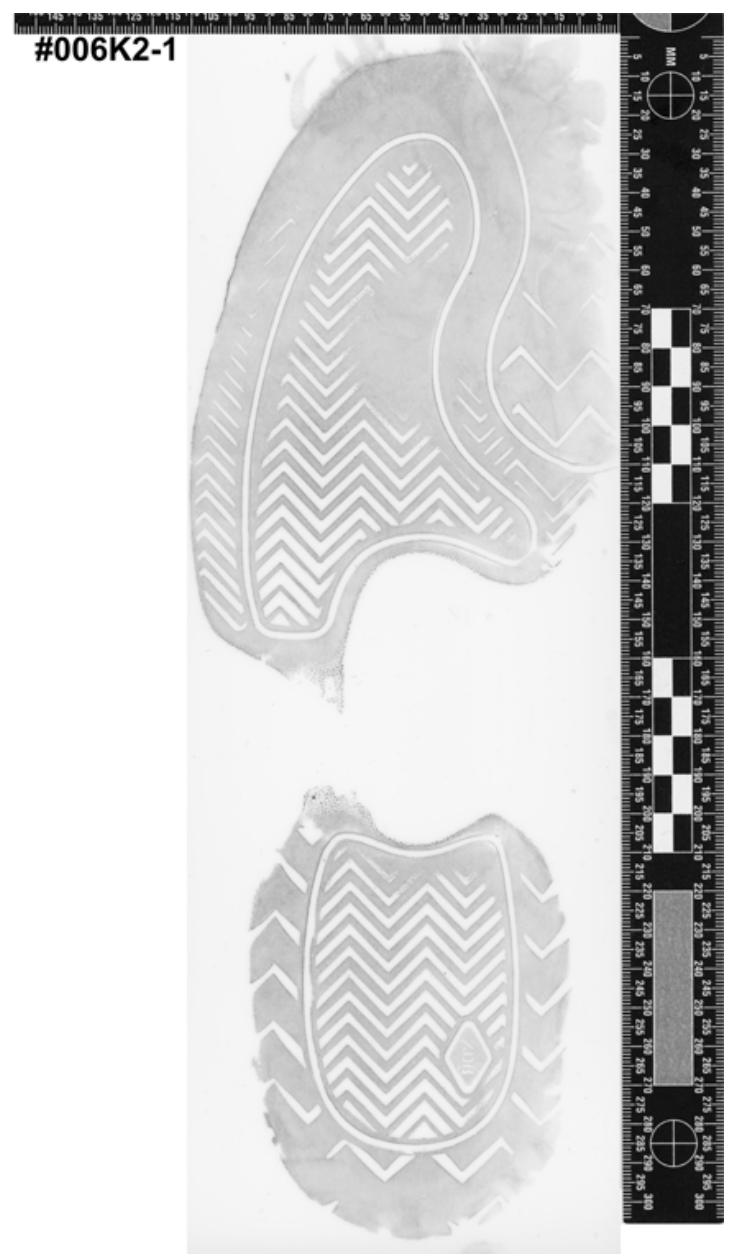

Figure D.26: Case 006: Sample Handiprint exemplar of known footwear 2, 006K2-1. 


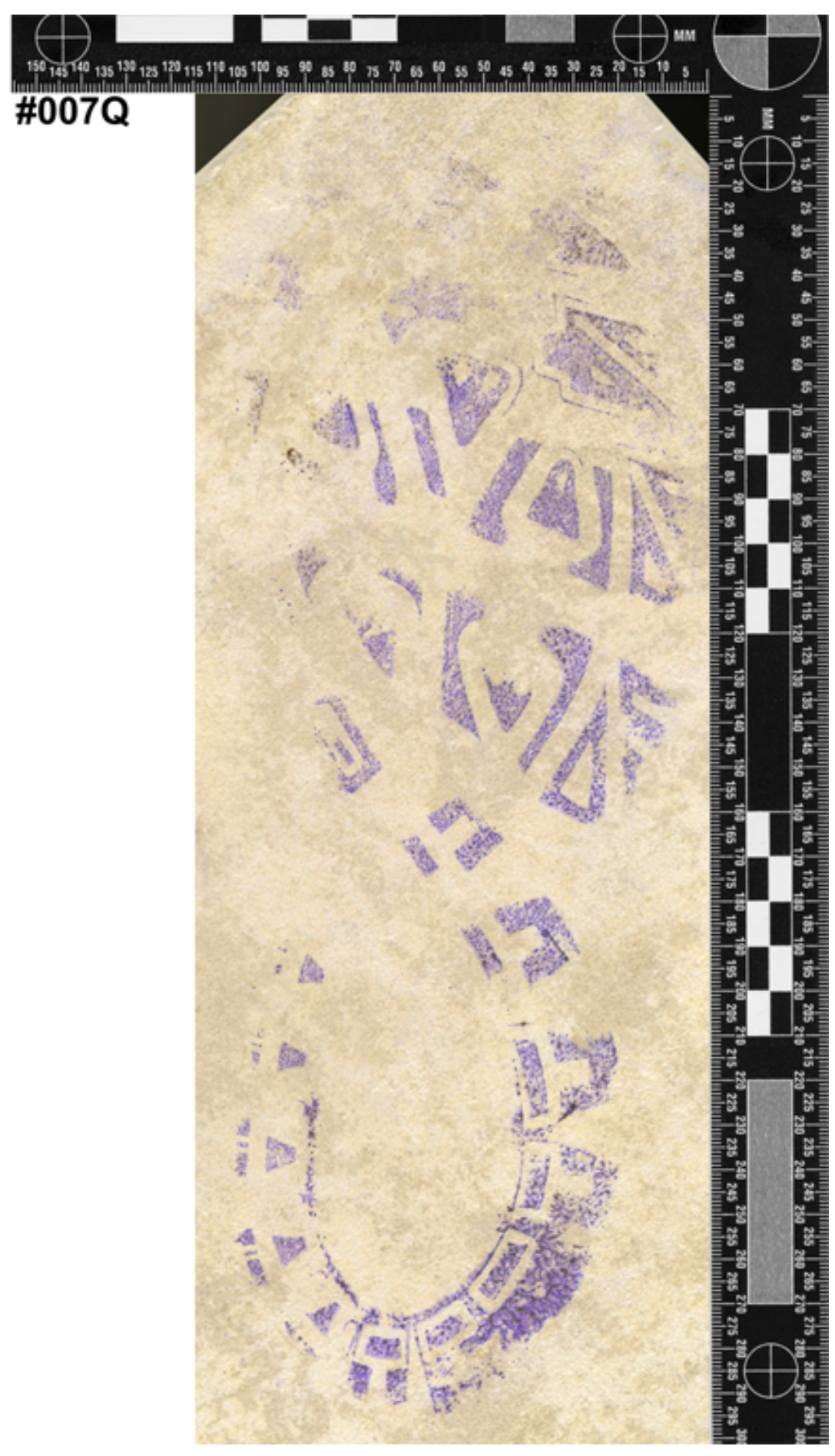

Figure D.27: Case 007: Questioned impression, 007Q. 


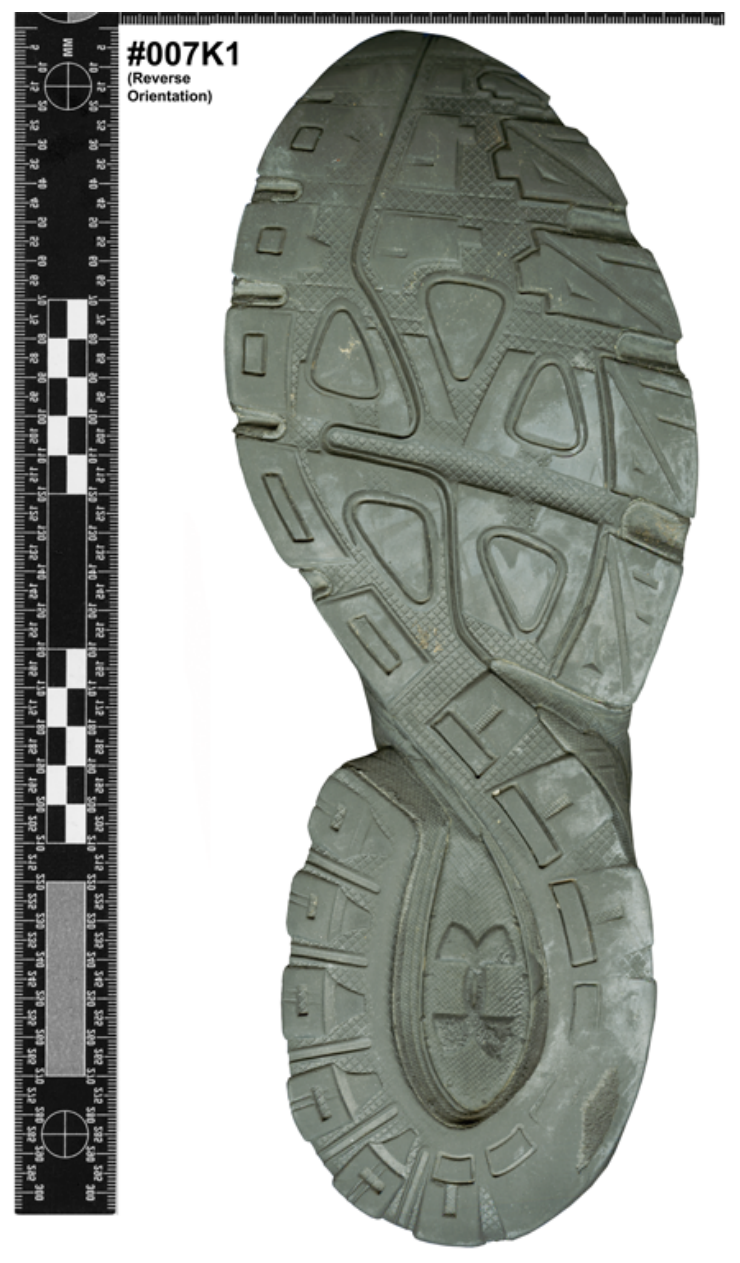

Figure D.28: Case 007: Outsole image of known footwear 1, 007K1.

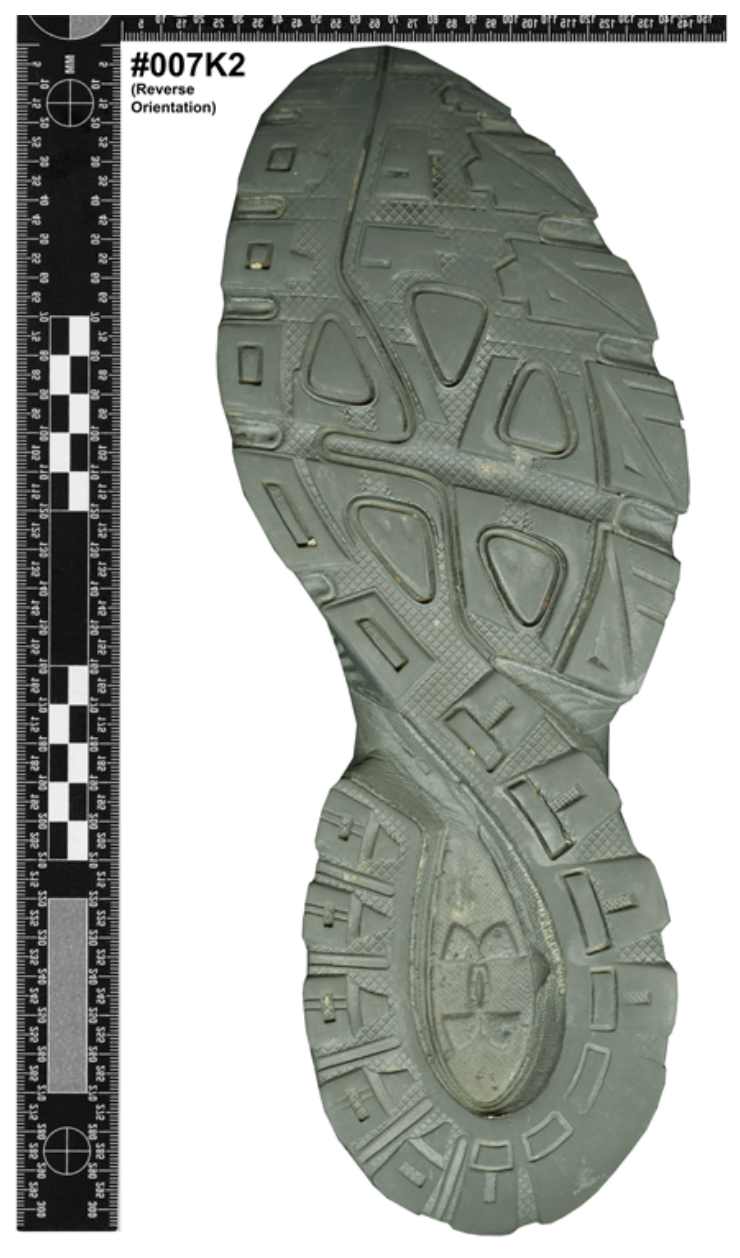

Figure D.29: Case 007: Outsole image of known footwear 2, 007K2. 


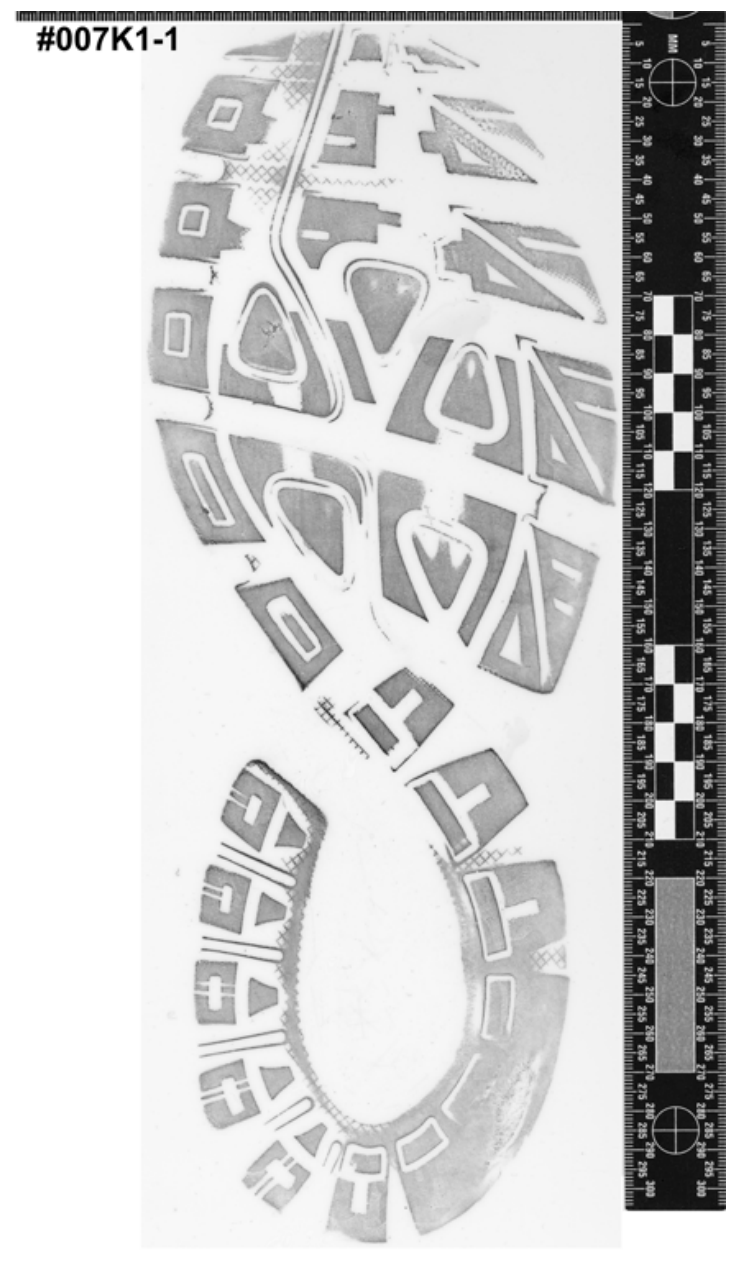

Figure D.30: Case 007: Sample Handiprint exemplar of known footwear 1, 007K1-1.

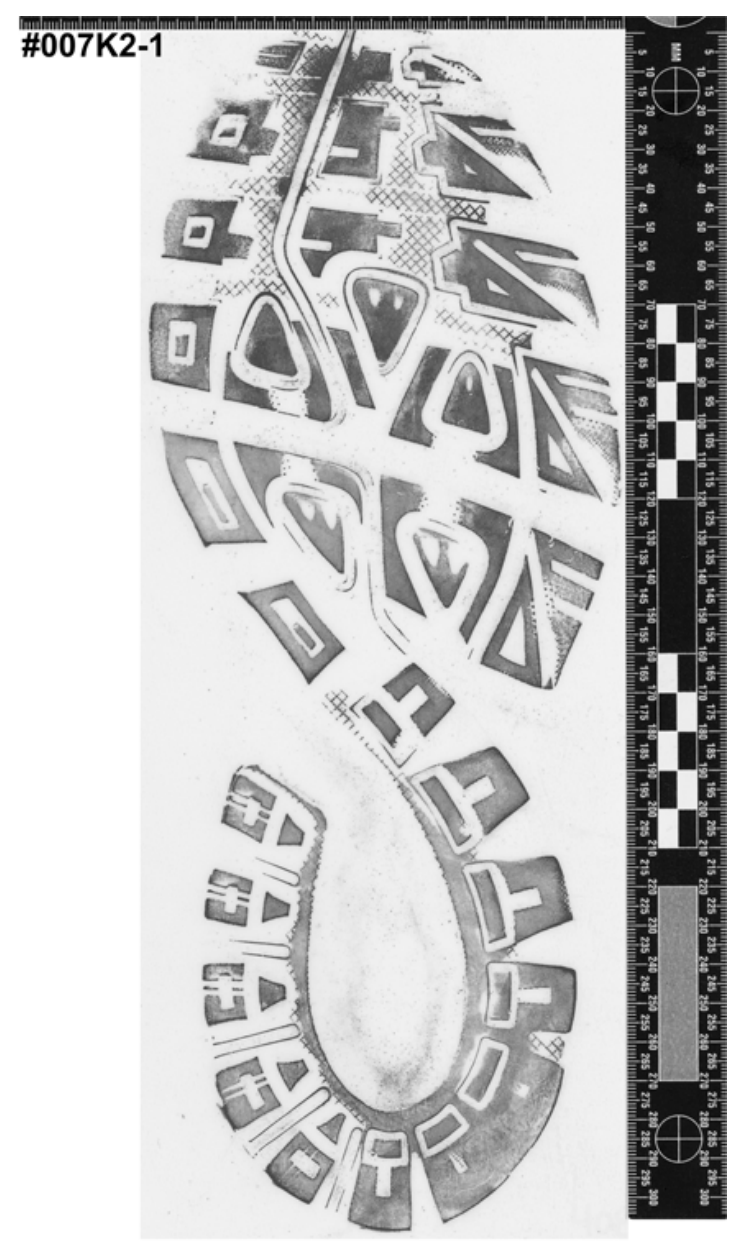

Figure D.31: Case 007: Sample Handiprint exemplar of known footwear 2, 007K2-1. 


\section{E. Background Survey}

\section{Academic and Practical Experiences Questionnaire}

\section{Academic Experience}

1. Are you currently enrolled in a forensic science program? If yes, please indicate your year of study.
$\square$ Yes, I am a freshman
$\square$ Yes, I am a senior
$\square$ Yes, I am a sophomore
$\square$ Yes, I am a graduate student
$\square$ Yes, I am a junior
$\square$ No, please specify:

2. Which of these areas of study have you successfully completed? Select all that apply.
$\square$ Introduction to forensic identification
$\square$ Fingerprint examination
$\square$ Firearms examination
$\square$ Crime scene investigation techniques
$\square$ Crime scene reconstruction
$\square$ Questioned document examination
$\square$ Trace evidence examination
$\square$ Criminalistics
$\square$ Forensic chemistry
$\square$ Forensic informatics

$\square$ Forensic photography

$\square$ Courtroom testimony

$\square$ Law and evidence

$\square$ Professional forensic communication

$\square$ Forensic quality assurance

$\square$ Forensic laboratory management

$\square$ Forensic casework practicum

$\square$ Forensic biology

$\square$ Computational forensics

$\square$ Other, please specify:

3. What is your area of concentration in pattern impression? Select all that apply.
$\square$ Footwear
$\square$ Fingerprint
$\square$ Firearms
$\square$ Handwriting analysis
$\square$ Toolmark
$\square$ Not applicable
$\square$ Other:

4. Do you have other areas of emphasis (other than pattern impression examination)? Select all that apply.
$\square$ Crime scene investigation
$\square$ Forensic biology
$\square$ Questioned documents examination
$\square$ Death investigation
$\square$ Trace evidence examination
$\square$ Toxicology
$\square$ Seized drugs analysis
$\square$ Arson and explosives investigation

$\square$ Other:

5. Please indicate the task(s) and frequency at which the task was performed in the laboratory or during mock crime scene(s) within the pattern impression discipline. Select all that apply.
$\square$ Collect impression evidence
seldom $\square-\square-\square-\square-\square$ very frequent
$\square \mathrm{N} / \mathrm{A}$
$\square$ Develop impression at crime scene
seldom $\square-\square-\square-\square-\square$ very frequent
$\square \mathrm{N} / \mathrm{A}$
$\square$ Enhance impression
seldom $\square-\square-\square-\square-\square$ very frequent
$\square \mathrm{N} / \mathrm{A}$
$\square$ Photograph impression evidence
seldom $\square-\square-\square-\square-\square$ very frequent
$\square \mathrm{N} / \mathrm{A}$
$\square$ Perform database searches
seldom $\square-\square-\square-\square-\square$ very frequent
$\square \mathrm{N} / \mathrm{A}$
$\square$ Examine/compare impression evidence
seldom $\square-\square-\square-\square-\square$ very frequent
$\square \mathrm{N} / \mathrm{A}$
$\square$ Other:
seldom $\square-\square-\square-\square-\square$ very frequent 
6. How many years of experience do you have in all forensic disciplines combined? This may include number of years in a FEPAC-accredited program, number of years in graduate school, number of months in internship, etc.

$\square$ None $\quad \square<1$ year $\quad \square$ 1-2 years $\square 3$-5 years $\square 6$-8 years $\square 8+$ years

7. Have you ever conducted a forensic footwear comparison/examination (either in a class or during a practical experience)?

$\square$ Yes $\square$ No

\section{Continuing Education and Training}

8. Did you receive your education in any of these disciplines? If so, please specify the corresponding level of education as well as your degree of concentration (i.e., major/minor). Select all that apply.
$\square$ Forensic science
$\square \quad \square$ Biology
$\square$ Criminology
$\square$ Law
$\square$ Chemistry
$\square$ Natural science
$\square$ Technology
$\square$ Other:

9. Have you participated in continuing education/training programs related to forensic science? If yes, please indicate the total number of training opportunities you have participated in.

$\square 0 \quad \square 1-2 \quad \square 3-4 \quad \square 4+$ times

9a. Please specify the discipline(s) in which you received training.
$\square$ Footwear examination
$\square$ Fingerprint examination
$\square$ Firearms examination
$\square$ Questioned document examination
$\square$ Toolmark examination
$\square$ Crime scene investigation

$\square$ Other:

9b. Please describe the type of training you received.
$\square$ IAI sponsored training
$\square$ Private/consultant-based course
$\square$ Conference workshops/seminar
$\square$ Vendor/supplier sponsored training
$\square$ Other:

9c. Which TWO course topics were the most useful?
$\square$ Advanced topics in specified discipline
$\square$ Skill advancement in photography
$\square$ Skill advancement in crime scene processing
$\square$ Courtroom preparation and testimony
$\square$ Skill advancement in laboratory processing
$\square$ Examination/comparison
$\square$ Other:

10. Have you taught any seminars or classes related to forensic science? This may include experience as a lead instructor, co-instructor, teaching assistant, etc.

$\square$ Yes $\square$ No 
10a. How many teaching opportunities have you had in relation to forensic science courses?
$\square 1-2$
$\square 3-4$
$\square 4+$ times

10b. Please specify the discipline(s) that you taught.
$\square$ Footwear examination
$\square$ Fingerprint examination
$\square$ Firearms examination
$\square$ Questioned document examination
$\square$ Toolmark examination
$\square$ Crime scene investigation
$\square$ Other:

10c. What type of course materials do you cover in these classes?
$\square$ Advanced topics in specified discipline
$\square$ Skill advancement in photography
$\square$ Skill advancement in crime scene processing
$\square$ Courtroom preparation and testimony
$\square$ Skill advancement in laboratory processing
$\checkmark$ Examination/comparison
$\square$ Other:

\section{Internship Experience and Quality Assurance}

11. Do you have practical internship experience in forensic science? Note: You may proceed to the next section if you do not have practical internship experience in forensic science.

$\square$ Yes $\square$ No

12. Please indicate the type of agency/organization where you completed your practical internship.
$\square$ State crime laboratory
$\square$ Police department
$\square$ County crime laboratory
$\square$ Office of Chief Medical Examiner
$\square$ Regional crime laboratory
$\square$ Private forensic laboratory
$\square$ Federal agency forensic laboratory
$\square$ University laboratory
$\square$ Non-profit organization
$\square$ Other:

13. Please indicate the discipline of focus during the internship experience.
$\square$ Footwear/tire track examination
$\square$ Forensic biology
$\square$ Crime scene investigation
$\square$ Death investigation
$\square$ Fingerprint/latent examination
$\square$ Toxicology
$\square$ Firearms and toolmarks examination
$\square$ Seized drugs analysis
$\checkmark$ Questioned documents examination
$\square$ Arson and explosives investigation
$\square$ Trace evidence examination
$\square$ Biometric systems/digital forensics
$\square$ Other:

14. Please specify the duties you performed at the internship site.
$\square$ Footwear analysis
$\square$ Evidence processing
$\square$ Crime scene processing
$\square$ Forensic photography
$\square$ Fingerprint evidence analysis
$\square$ Research/quality assurance project(s)
$\square$ Firearms and toolmarks analysis
$\square$ Observation only
$\square$ Examination/comparison of trace evidence
$\square$ Database entry/searches

$\square$ Other:

15. Was the discipline/section/unit accredited?
$\square$ Yes
$\square$ No
$\square$ Do not know

16. Please specify the accrediting organization(s) of your internship site, if applicable/known. 
17. Did the agency perform analyses in the following areas? Select all that apply.

$\square$ Footwear/tire track examination

$\square$ Forensic biology

$\square$ Crime scene investigation

$\square$ Death investigation

$\square$ Fingerprint/latent examination

$\square$ Toxicology

$\square$ Firearms and toolmarks examination

$\square$ Seized drugs analysis

$\square$ Questioned documents examination

$\square$ Arson and explosives investigation

$\square$ Trace evidence examination

$\square$ Biometric systems/digital forensics

$\square$ Other:

\section{Research and Publications}

18. Have you conducted any pattern impression-related research? Note: You may proceed to the next section if you do not have research experience or publications in pattern impression evidence.

$\square$ Yes $\square$ No

18a. Please specify the type of pattern evidence on which you conducted your research.

19. Have you published any works on any topic of pattern impression evidence?

$\square$ Yes $\square$ No

19a. Please specify the type of pattern evidence on which you published your work.

19b. Please indicate the type of work(s) published. Select all that apply.

$\square$ Book

$\square$ Chapter in a book

$\square$ Experimental research

$\square$ Case study

$\square$ Literature review

$\square$ Thesis/dissertation

$\square$ Other: 


\section{F. GUI}

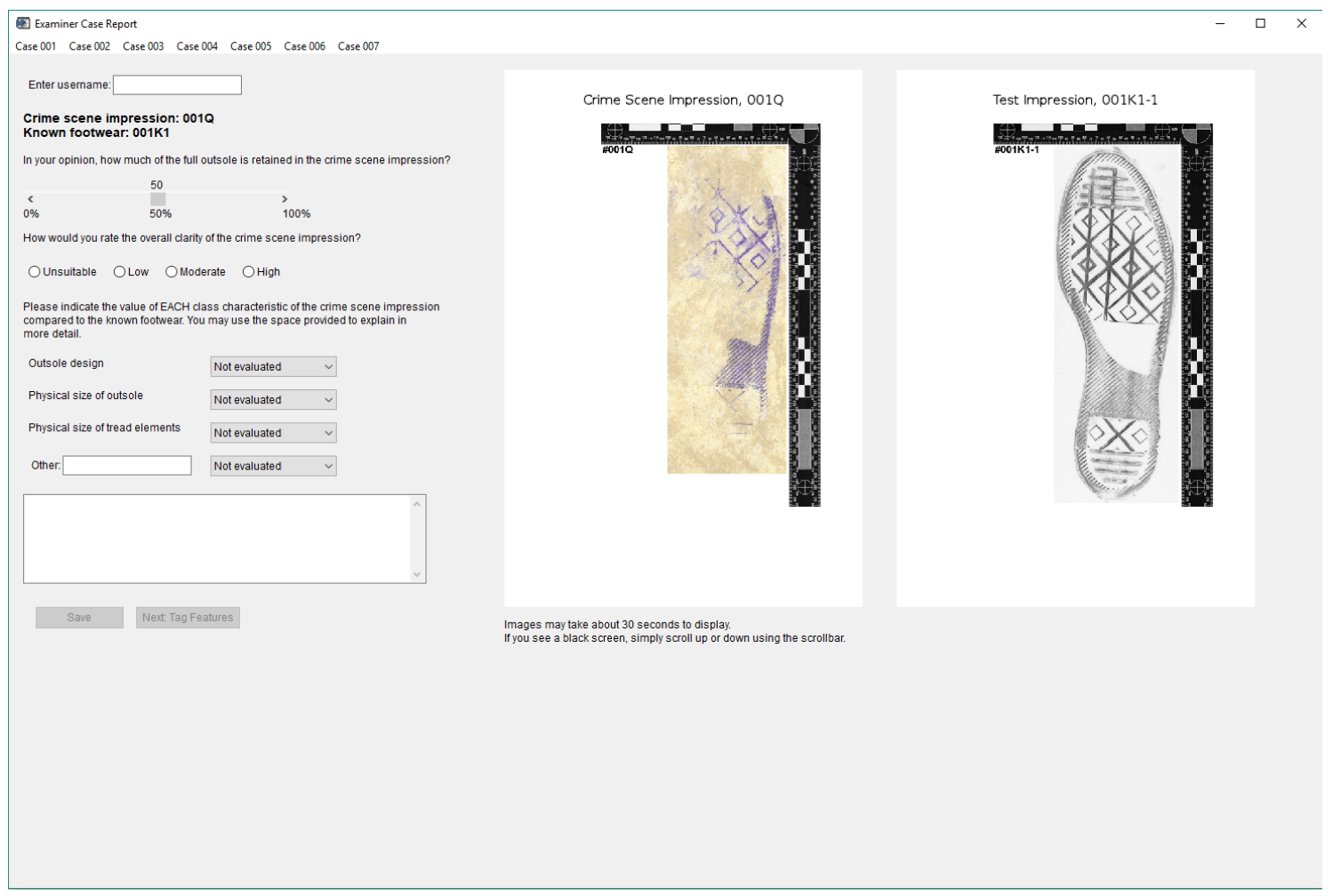

Figure F.1: Page 1 contains questions regarding assessment of crime scene impression totality and clarity, and the value of class characteristics. 


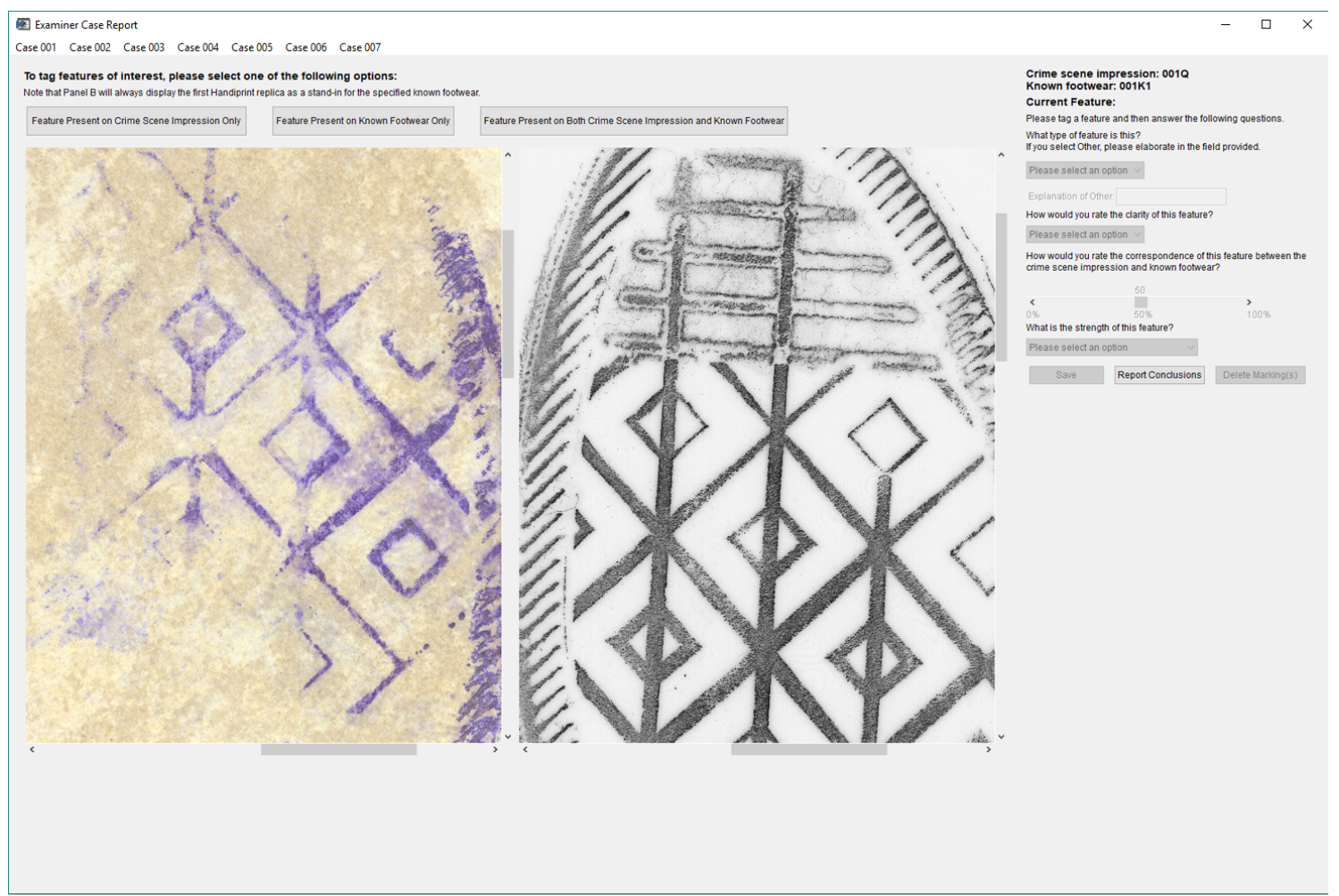

Figure F.2: Page 2 allows examiners to mark features found in the crime scene impression, known footwear, or both. 


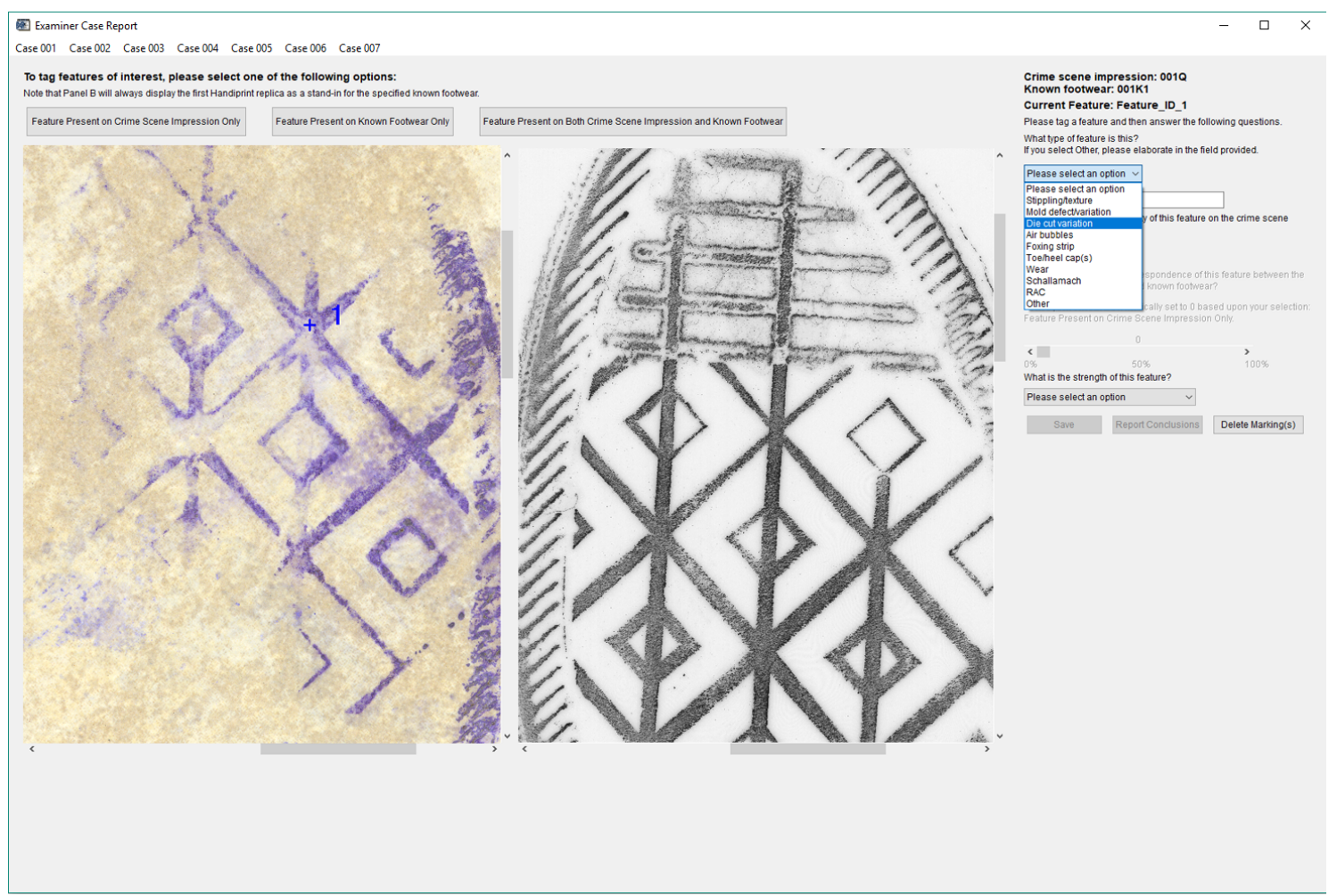

Figure F.3: After marking a feature, examiners must specify the type of feature, as well as comment on the feature clarity, similarity between the crime scene impression and known footwear (where applicable), and finally, value with regard to their ultimate conclusion for the case. 


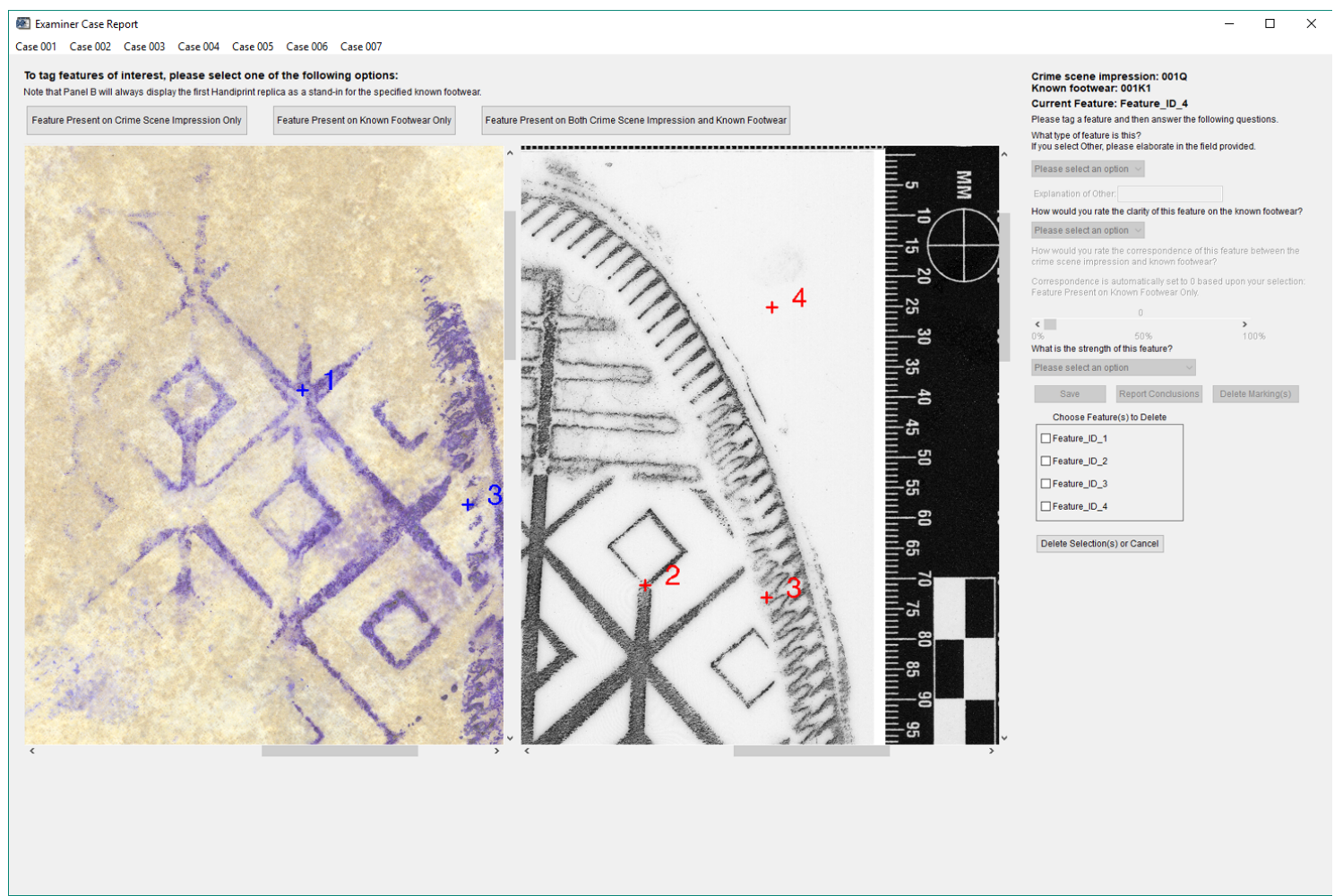

Figure F.4: In the event that an examiner mistakenly marked a feature, he or she may delete said feature. 


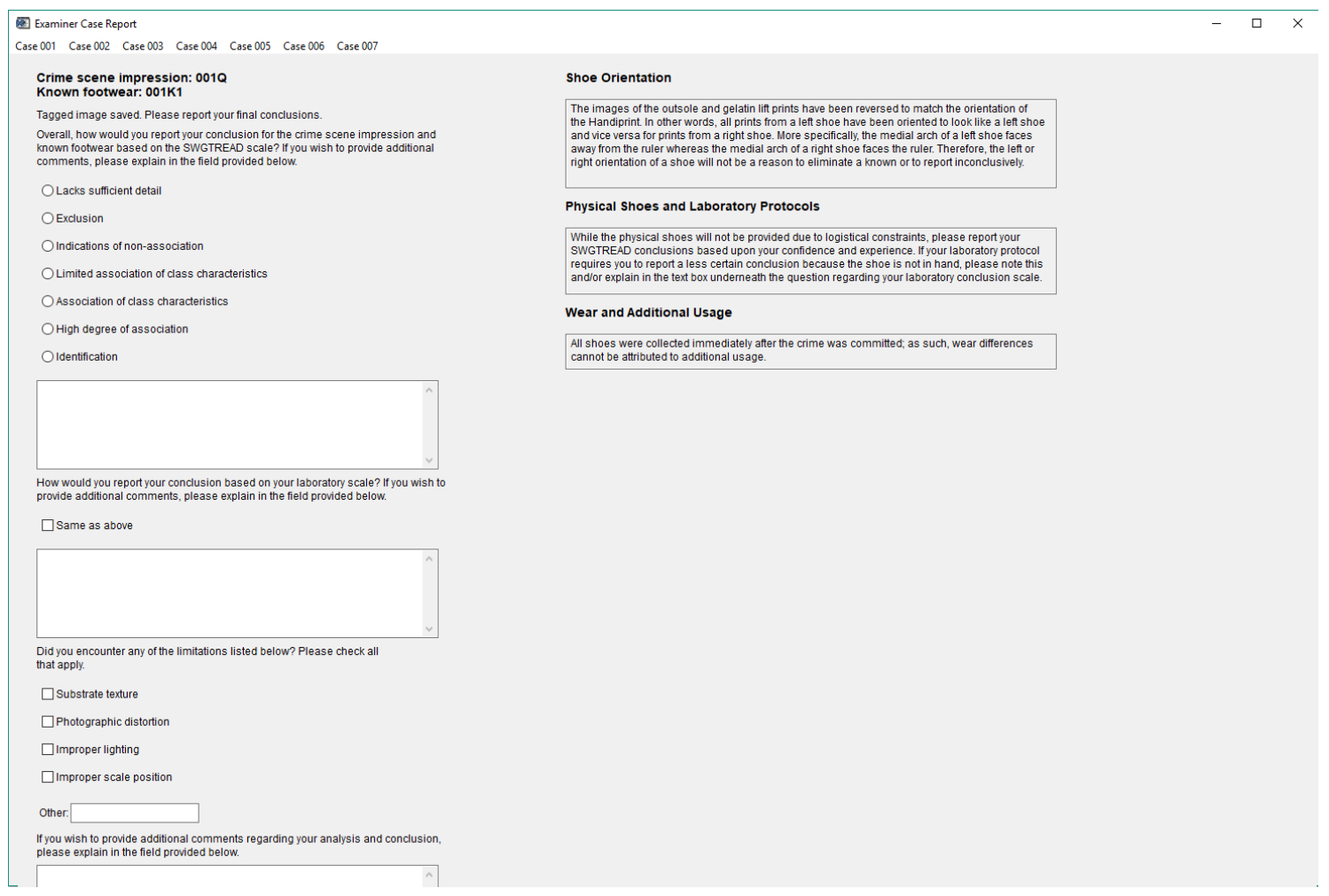

Figure F.5: The examiner then reports his or her final conclusions regarding the level of association between the questioned impression and the known(s). He or she may also opt to elaborate on the differences between the SWGTREAD and laboratory conclusion scales (if necessary). 


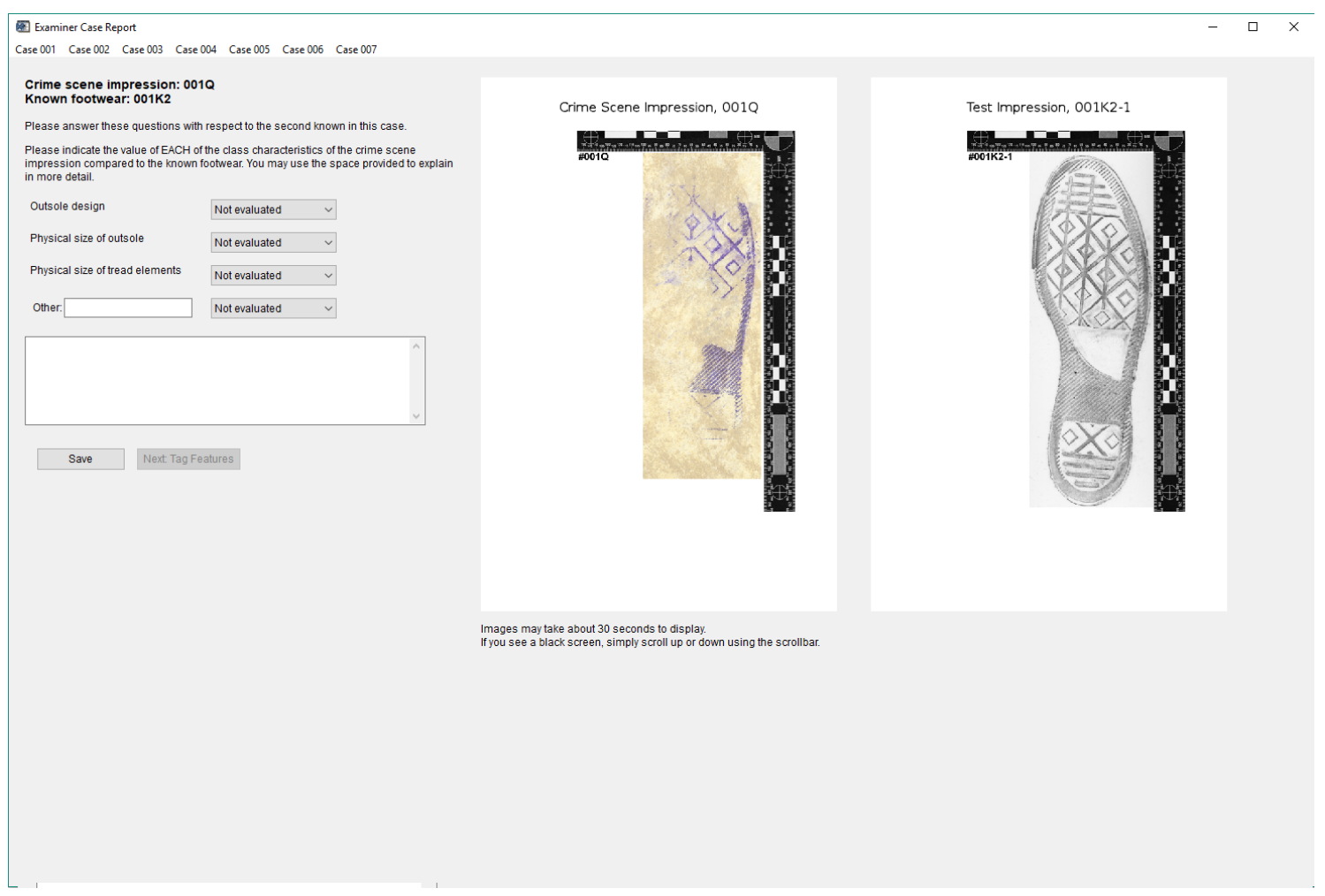

Figure F.6: For cases with two known exemplars, participants were then prompted to answer questions regarding the second exemplar, without repeating their responses for the questioned impression. 
Table G.1: An illustrative example showing the academic and research background information obtained from novice participants (note that the following table does not reflect the answers provided by any specific participant, and is only illustrative in nature). $G S$ $=$ Graduate student, $B S=$ Bachelor of Science, $F S=$ Forensic science, $Y=Y e s, C S I$ = Crime scene investigation, $Q D E=$ Questioned document examination, $S A=$ Skill advancement.

\begin{tabular}{|c|c|}
\hline Participant & Alphanumeric User ID \\
\hline \multicolumn{2}{|l|}{ Overall Experience: } \\
\hline Year of Study & GS \\
\hline Combined Experience in Forensic Science (years) & $3-5$ \\
\hline Experience in Footwear Examination (years) & $<1$ \\
\hline Highest Education Level & BS \\
\hline Major Concentration & FS \\
\hline \multicolumn{2}{|l|}{ Major Concentration 2} \\
\hline Minor Concentration & Biology \\
\hline \multicolumn{2}{|l|}{ Minor Concentration 2} \\
\hline \multicolumn{2}{|l|}{ Areas of Emphasis: } \\
\hline Pattern Impression & Fingerprint \\
\hline CSI & $\mathrm{Y}^{\mathrm{t}}$ \\
\hline Questioned Document Examination & $\mathrm{Y}$ \\
\hline Trace Evidence Examination & $\mathrm{Y}$ \\
\hline Seized Drug Analysis & $\mathrm{Y}$ \\
\hline \multicolumn{2}{|l|}{ Forensic Biology } \\
\hline \multicolumn{2}{|l|}{ Death Investigation } \\
\hline \multicolumn{2}{|l|}{ Toxicology } \\
\hline \multicolumn{2}{|l|}{ Arson and Explosives Investigation } \\
\hline \multicolumn{2}{|l|}{ Courses Taken: } \\
\hline Introduction to Forensic Identification & $\mathrm{Y}$ \\
\hline Fingerprint Examination & $\mathrm{Y}$ \\
\hline Firearms Examination & $\mathrm{Y}$ \\
\hline CSI Techniques & $\mathrm{Y}$ \\
\hline Crime Scene Reconstruction & $\mathrm{Y}$ \\
\hline \multicolumn{2}{|l|}{ Questioned Document Examination } \\
\hline Trace Evidence Examination & $\mathrm{Y}$ \\
\hline Criminalistics & $\mathrm{Y}$ \\
\hline Forensic Chemistry & $\mathrm{Y}$ \\
\hline Forensic Informatics & $\mathrm{Y}$ \\
\hline Forensic Photography & $\mathrm{Y}$ \\
\hline Courtroom Testimony & $\mathrm{Y}$ \\
\hline Law and Evidence & $\mathrm{Y}$ \\
\hline Professional Communications & $\mathrm{Y}$ \\
\hline Forensic Quality Assurance & $\mathrm{Y}$ \\
\hline Forensic Laboratory Management & $\mathrm{Y}$ \\
\hline \multicolumn{2}{|l|}{ Forensic Casework Practicum } \\
\hline Forensic Biology & $\mathrm{Y}$ \\
\hline \multicolumn{2}{|l|}{ Computational Forensics } \\
\hline Other & \\
\hline
\end{tabular}




\section{G. Sample Data Tables}

\begin{tabular}{|c|c|}
\hline \multicolumn{2}{|c|}{ Tasks Performed in Relation to Impression Evidence? } \\
\hline Collection & 4 \\
\hline Development & 5 \\
\hline Enhancement & 3 \\
\hline Photography & 5 \\
\hline Database Searches & 5 \\
\hline Examination and/or Comparison & 4 \\
\hline \multicolumn{2}{|l|}{ Research and Publication: } \\
\hline \multicolumn{2}{|l|}{ Research Experience } \\
\hline \multicolumn{2}{|l|}{ Research Discipline } \\
\hline \multicolumn{2}{|l|}{ Publication } \\
\hline \multicolumn{2}{|l|}{ Continuing Education: } \\
\hline Participation (No. of times) & $1-2$ \\
\hline Type 1 & SA in crime scene processing \\
\hline Type 2 & $\mathrm{SA}$ in laboratory processing \\
\hline Subject 1 & Fingerprint examination \\
\hline Subject 2 & Evidence photography \\
\hline Sponsor / Organization & Conference workshop/seminar \\
\hline Teaching Opportunities (No. of times): & $>4$ \\
\hline Type 1 & SA in crime scene processing \\
\hline Type 2 & SA in laboratory processing \\
\hline \multicolumn{2}{|l|}{ Disciplines Taught: } \\
\hline \multicolumn{2}{|l|}{ Footwear Examination } \\
\hline \multicolumn{2}{|l|}{ Firearms Examination } \\
\hline \multicolumn{2}{|l|}{ Toolmark Examination } \\
\hline \multirow{2}{*}{\multicolumn{2}{|c|}{$\begin{array}{l}\text { FDE } \\
\text { QDE }\end{array}$}} \\
\hline & \\
\hline CSI & $\mathrm{Y}$ \\
\hline Other & Forensic photography \\
\hline \multicolumn{2}{|l|}{ Internship Experience: } \\
\hline Interning Agency & Police department \\
\hline Agency Accreditation & Do not know \\
\hline \multicolumn{2}{|l|}{ Forensic Services Offered: } \\
\hline Footwear/Tire Track Examination & $\mathrm{Y}$ \\
\hline Crime Scene Investigation & $\mathrm{Y}$ \\
\hline Fingerprint/Latent Examination & $\mathrm{Y}$ \\
\hline Firearms and Toolmark Examination & $\mathrm{Y}$ \\
\hline Questioned Document Examination & $\mathrm{Y}$ \\
\hline Trace Evidence Examination & $\mathrm{Y}$ \\
\hline \multicolumn{2}{|l|}{ Forensic Biology } \\
\hline \multicolumn{2}{|l|}{ Death Investigation } \\
\hline \multicolumn{2}{|l|}{ Toxicology } \\
\hline \multirow{2}{*}{\multicolumn{2}{|c|}{$\begin{array}{l}\text { Seized Drugs Analysis } \\
\text { Arson and Explosives Investigation }\end{array}$}} \\
\hline & \\
\hline \multicolumn{2}{|l|}{ Biometric Systems / Digital Forensics } \\
\hline Discipline of Focus & CSI \\
\hline \multicolumn{2}{|l|}{ Duties Performed: } \\
\hline \multicolumn{2}{|l|}{ Footwear analysis } \\
\hline Crime Scene Processing & $\mathrm{Y}$ \\
\hline Fingerprint Evidence Analysis & $\mathrm{Y}$ \\
\hline Firearms and Toolmarks Analysis & \\
\hline Trace Evidence Analysis & \\
\hline Evidence Processing & $\mathrm{Y}$ \\
\hline Forensic Photography & $\mathrm{Y}$ \\
\hline Research / Quality Ássurance & \\
\hline Observation Only & \\
\hline Database Entry / Search & $\mathrm{Y}$ \\
\hline
\end{tabular}

${ }^{1} 0=$ Never, $1=$ Seldom, $2=$ Rarely, $3=$ Sometimes, $4=$ Frequently, $5=$ Very frequently 
Table G.2: A figurative example showing a fictitious participant's overall report on each questioned-to-known comparison.

\begin{tabular}{|c|c|c|c|c|c|c|c|c|c|c|c|c|}
\hline Case & $001 \mathrm{~K} 1$ & $001 \mathrm{~K} 2$ & $002 \mathrm{~K} 1$ & $003 \mathrm{~K} 1$ & $003 \mathrm{~K} 2$ & $004 \mathrm{~K} 1$ & $004 \mathrm{~K} 2$ & $005 \mathrm{~K} 1$ & $006 \mathrm{~K} 1$ & $006 \mathrm{~K} 2$ & $007 \mathrm{~K} 1$ & $007 \mathrm{~K} 2$ \\
\hline Outsole Retention in CS Impression (\%) & 50 & 50 & 20 & 85 & 85 & 15 & 15 & 30 & 40 & 40 & 60 & 60 \\
\hline Clarity of Crime Scene Impression ${ }^{2}$ & M & M & $\mathrm{L}$ & $\mathrm{H}$ & $\mathrm{H}$ & $\mathrm{L}$ & $\mathrm{L}$ & $\mathrm{L}$ & $\mathrm{L}$ & $\mathrm{L}$ & M & M \\
\hline \multicolumn{13}{|l|}{ Value of Class Characteristics ${ }^{3}$} \\
\hline Outsole Design & 1 & 1 & 3 & 1 & 3 & 2 & 1 & 2 & 1 & 1 & 1 & 1 \\
\hline Physical Size (Outsole) & 1 & 2 & 1 & 2 & 3 & 0 & 1 & 1 & 3 & 3 & 2 & 1 \\
\hline Physical Size (Design) & 2 & 2 & 1 & 2 & 2 & 2 & 1 & 1 & 3 & 3 & 2 & 1 \\
\hline Other: & 0 & 0 & 0 & 0 & 0 & 0 & 0 & 0 & 0 & 0 & 0 & 0 \\
\hline \multicolumn{13}{|l|}{ Limitations and Interferences: 4} \\
\hline Substrate Texture & $\mathrm{Y}$ & $\mathrm{Y}$ & $\mathrm{Y}$ & & Y & & & $\mathrm{Y}$ & $\mathrm{Y}$ & & $\mathrm{Y}$ & $\mathrm{Y}$ \\
\hline Improper Lighting & & & & $\mathrm{Y}$ & & & & $\mathrm{Y}$ & & & & \\
\hline $\begin{array}{l}\text { Improper Scale Position } \\
\text { Other }\end{array}$ & & & & $\mathrm{Y}$ & & & & & $\mathrm{Y}$ & & & \\
\hline Case Difficulty $^{5}$ & 3 & 3 & 3 & 3 & 3 & 2 & 2 & 3 & 3 & 3 & 3 & 3 \\
\hline SWGTREAD Conclusion ${ }^{6}$ & 5 & 3 & 1 & 3 & 4 & 2 & 5 & 1 & 1 & 1 & 4 & 5 \\
\hline
\end{tabular}

${ }^{2} \mathrm{~L}=$ Low, $\mathrm{M}=$ Moderate, $\mathrm{H}=$ High

$30=$ Not evaluated, $1=$ Value for association, $2=$ Value for exclusion, $3=$ Insufficient detail

${ }^{4} \mathrm{Y}=$ Yes

51 = Easy, 2 = Moderate, $3=$ Challenging

61 = Lacks sufficient detail, $2=$ Exclusion, $3=$ Indications of non-association, $4=$ Limited association of class characteristics, $5=$ Association of class characteristics, $6=$ High degree of association, $7=$ Identification

Table G.3: An illustrative table showing the feature type, clarity, and similarity of selected features marked by a respondent.

\begin{tabular}{|c|c|c|c|c|c|c|c|c|c|c|c|c|}
\hline Case & 001K1 & 001K1 & $001 \mathrm{~K} 2$ & $001 \mathrm{~K} 2$ & $002 \mathrm{~K} 1$ & $003 \mathrm{~K} 1$ & $003 \mathrm{~K} 1$ & 003K2 & 003K2 & $004 \mathrm{~K} 1$ & 004K1 & 004K1 \\
\hline Feature No. & 1 & 2 & 1 & 2 & 1 & 1 & 2 & 1 & 2 & 1 & 2 & \\
\hline Feature Type ${ }^{7}$ & 9 & 9 & 9 & 9 & 7 & 7 & 1 & 9 & 10 & 9 & 9 & 9 \\
\hline Clarity of Feature 8 & 1 & 3 & 2 & 2 & 1 & 1 & 1 & 1 & 1 & 2 & 3 & 2 \\
\hline Correspondence of Feature (\%) & 90 & 40 & 50 & 50 & 15 & 40 & 30 & 40 & 40 & 60 & 80 & 60 \\
\hline Strength of Feature ${ }^{9}$ & 3 & 3 & 1 & 1 & 3 & 2 & 2 & 1 & 2 & 1 & 1 & 1 \\
\hline
\end{tabular}

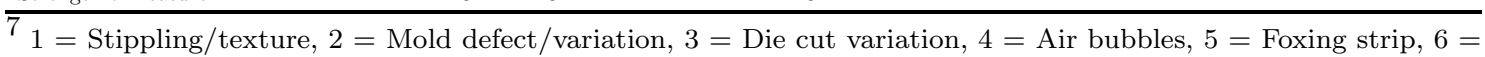
Toe/heel $\operatorname{cap}(\mathrm{s}), 7=$ Wear, $8=$ Schallamach, $9=\mathrm{RAC}, 10=$ Other

81 = Low, 2 = Moderate, $3=$ High

9 Read as "Supports ..." $1=$ Exclusion, $2=$ Non-association, $3=$ Limited association, $4=$ Association, $5=$ High degree of association, $6=$ Identification 